\title{
Analytical and variational numerical methods for unstable miscible displacement flows in porous media
}

\author{
Guglielmo Scovazzi ${ }^{\mathrm{a}, *}$, Mary F. Wheeler ${ }^{\mathrm{b}}$, Andro Mikelićc ${ }^{\mathrm{c}}$, Sanghyun Lee ${ }^{\mathrm{b}}$ \\ ${ }^{a}$ Department of Civil E Environmental Engineering, Duke University, Room 121 Hudson Hall, P.O. Box 90287, Durham, NC 27708-0287, USA \\ ${ }^{b}$ The Center for Subsurface Modeling, The Institute for Computational Engineering and Sciences, The University of Texas at Austin, Austin, Texas \\ 78712, USA \\ ${ }^{c}$ Université de Lyon, CNRS UMR 5208, Université Lyon 1, Institut Camille Jordan, 43, blvd. du 11 novembre 1918, 69622 Villeurbanne Cedex, \\ France
}

\begin{abstract}
The miscible displacement of one fluid by another in a porous medium has received considerable attention in subsurface, environmental and petroleum engineering applications. When a fluid of higher mobility displaces another of lower mobility, unstable patterns - referred to as viscous fingering - may arise. Their physical and mathematical study has been the object of numerous investigations over the past century. The objective of this paper is to present a review of these contributions with particular emphasis on variational methods. These algorithms are tailored to real field applications thanks to their advanced features: handling of general complex geometries, robustness in the presence of rough tensor coefficients, low sensitivity to mesh orientation in advection dominated scenarios, and provable convergence with fully unstructured grids.

This paper is dedicated to the memory of Dr. Jim Douglas Jr., for his seminal contributions to miscible displacement and variational numerical methods.

Keywords: Miscible displacement, viscous fingering, flow instabilities, Hele-Shaw, variational methods, numerical simulation, Galerkin methods
\end{abstract}

\section{Introduction}

Two fluids are miscible when they can be mixed together in all proportions and all mixtures remain single phase. Understanding the role of miscibility has been a major concern in developing efficient enhanced oil recovery (EOR) processes. Here, miscible EOR involves injection of a solvent at certain wells in a petroleum reservoir, with the intention of displacing resident oil to other production wells. Since early 1950s, the search for an effective and economical solvent along with development and field testing of miscible-flood processes has received considerable attention. The economics of the process can be costly, because the chemicals it requires are expensive and the performance of the displacement may involve significant variability. Later on it was realized that the impact of the early miscible displacement investigations extended to a much broader range of applications, which included contaminant transport, bioremediation, carbon dioxide $\left(\mathrm{CO}_{2}\right)$ dissolution in saline aquifers, hydraulic fracturing, and various problems in the biomedical field.

Mathematically, the miscible displacement process is described by a strongly coupled, highly nonlinear system of nonlinear, parabolic, convection-dominated (near-hyperbolic) partial differential equations for each of the chemical components. By summing the component equations, an equation for the pressure is obtained, which is also nonlinear, and can be classified as elliptic or parabolic, depending on whether the system is incompressible or compressible. Often the coupled nonlinear dynamics arising from miscible displacement problems may result in complex chaotic

\footnotetext{
${ }^{*}$ Corresponding author

Email addresses: guglielmo.scovazzi@duke. edu (Guglielmo Scovazzi), mf w@ices . utexas .edu (Mary F. Wheeler), mikelic@univ-lyon1.fr (Andro Mikelić), shlee@ices . utexas .edu (Sanghyun Lee)
} 
phenomena, frequently referred to as viscous fingering. Needless to say that the numerical simulation of viscous fingering phenomena can pose a considerable challenge, especially if accurate and robust algorithms in complex geometry are sought.

In this review, we will concentrate on miscible single-phase flow. From a numerical algorithmic perspective, we will emphasize variational methods since they provide a general framework that encompasses both continuous and discontinuous Galerkin methods as well as finite volumes and finite differences. In addition, the literature of Galerkin discretizations is much scarcer than that of finite volume/difference and spectral methods for miscible displacement. Variational methods provide two important features in practical computational engineering applications: 1) the ability to handle very complex geometries with unstructured grids with rough tensor coefficients; 2) the flexibility of imposing general boundary conditions. We believe that this is where the value of this survey stands.

We begin our discussion by formulating the physical model of miscible displacement and describing the key features of related viscous and gravitational fingering flow instabilities. The latter are triggered by highly varying permeabilities and adverse density and viscosity ratios when solute is injected in a porous medium. Next, we describe the computational challenges that need to be overcome in order to achieve high fidelity simulations, among which, coupling of nonlinear equations, solvers, grid sensitivity of numerical results, local and global conservation properties, compatibility principles between flow and transport equations, multiscale algorithms and mesh adaptivity. We report a chronological account of the main computational accomplishments that have enabled and contributed to the fidelity of miscible displacement computations in general and viscous fingering simulations in particular. In the presentation of main algorithms, we decided to separate discretizations for flow and transport as often times these discretizations can be used interchangeably. We present computations of miscible displacement flows found in subsurface engineering applications, among which EOR, $\mathrm{CO}_{2}$ sequestration, bioremediation, and hydraulic fracturing. Unstable miscible displacement represents an important phenomena contributing to the complexity of these systems.

To our best knowledge, we have strived to be inclusive of all the recent and past advances in this rapidly evolving field. We apologize to the authors whose contribution we may have inadvertently omitted. For example, we are aware that much of the results discussed here have also been applied to immiscible displacement calculations, but, due to the breadth of this topic, we have limited our discussion to miscible displacement.

\section{Theoretical and analytical framework}

\subsection{Governing equations}

We consider the equations for a multi-component fully miscible compressible flow in which only one liquid phase is present [1, 2, 3]. In the incompressible limit, this model is one of the most commonly used to study viscous fingering problems, and a large literature is available: see for example Tan and Homsy [4, 5, 6, 7], Manickam and Homsy [8], Homsy [9], Zimmerman and Homsy [10, 11, 12], Araktingi and Orr Jr [13], Chen and Meiburg [14, 15], Meiburg and Chen [16], Riaz and Tchelepi [17, 18], Tchelepi et al. [19], Saffman and Taylor [20], Saffman [21], Chang and Slattery [22], Raghavan and Marsden [23], Shikaze et al. [24], Schincariol et al. [25], Zhang and Schwartz [26], Schincariol and Schwartz [27], Cuthiell et al. [28], Sharma et al. [29], Holm [30] and references therein. The literature is much scarcer in the compressible case, but we present governing equations that encompass both compressible and incompressible displacement flow.

\subsubsection{General form}

The general equations for multiphase multicomponent reactive subsurface flows were introduced more than two decades ago, and are extensively used for simulations in hydrology and petroleum engineering. Classic references are the well-known monographs by Bear and Bachmat [31] and Nield and Bejan [32]. In many applications considered here, such as, for example, hazardous waste and $\mathrm{CO}_{2}$ sequestration, only single phase multicomponent reactive flows are considered. The corresponding equations are presented in detail in the review article of Kolditz and collaborators [33] (see also [34] and [1], Chapter 2). Most references found in the literature treat the case of two-component miscible displacement (see [35] and [36] for a detailed discussion of important specific cases). Furthermore, we restrict our considerations to isothermal flows.

We start with the general setting of single phase multicomponent systems: Let $n_{d}$ be the number of spatial dimensions and $\Omega$ an open set in $\mathbb{R}^{n_{d}}$ with boundary $\Gamma=\partial \Omega$ and outward-pointing normal $\mathbf{n}$. In what follows, vector fields 
will be denoted by boldface fonts. For example, $\mathbf{x} \in \Omega$ is the position vector and $\alpha$ a scalar. The mass conservation equation can be written in terms of the mass fractions $c_{i}$ as

$$
\partial_{t}\left(\phi \rho c_{i}\right)+\nabla \cdot\left(\rho \mathbf{u} c_{i}\right)=\nabla \cdot\left(\phi \rho \mathbf{D}(\mathbf{u}) \nabla c_{i}\right)+q_{i}, \quad i=1, \ldots, N_{c},
$$

where $\phi$ is the porosity of the medium, $\rho$ is the bulk fluid density, $\rho_{i}$ is the density of component $i\left(i=1,2, \ldots, N_{c}\right)$, $\mathbf{u}$ is the flow velocity (i.e, $\phi^{-1} \mathbf{u}$ is the macroscopic upscaled fluid velocity) and $\mathbf{D}$ is the hydrodynamic dispersion tensor, quantifying mass diffusion and mechanical dispersion effects. Here, $q_{i}$ is the mass source/sink term relative to the $i$-th component, and we also define $q=\sum_{i=1}^{N_{c}} q_{i}$. Namely,

$$
q_{i}:=\left\{\begin{array}{l}
\hat{q}_{i}(\text { datum }), \text { if } q>0, \\
c_{i} \hat{q} \text { (resident concentration sink), if } q \leq 0 .
\end{array}\right.
$$

Note also that, by definition,

$$
\sum_{j=1}^{N_{c}} c_{j}=1 .
$$

Remark 1. In practical reservoir applications, special wells can be engineered to selectively accept only some of the components in a mixture. These configurations can be mathematically modeled defining well sinks in which $q_{i}=\tilde{c}_{i} q$, with $\tilde{c}_{i}(\mathbf{x}) \leq c_{i}(\mathbf{x})$. We consider these situations beyond the scope of our discussion.

The bulk fluid density $\rho$ is a function of the mass fractions $c_{i}$ 's as different components have different densities, according to

$$
\rho=\frac{1}{\sum_{i=1}^{N_{c}} \frac{c_{i}}{\rho_{i}}} .
$$

A general expression for $\mathbf{D}(\mathbf{u})$ often used in the literature is

$$
\mathbf{D}(\mathbf{u}):=\left(\lambda D_{0}+\alpha_{T}|\mathbf{u}|\right) \mathbf{I}+\left(\alpha_{L}-\alpha_{T}\right) \frac{\mathbf{u} \otimes \mathbf{u}}{|\mathbf{u}|},
$$

where $|\mathbf{u}|$ is the Euclidean norm of the velocity vector $\mathbf{u}$. We refer the reader to Section 2.4] and [31], [2] and [37] for more details.

Remark 2. The authors of [38] proposed a different model for the dispersion tensor, which was set equal to $\phi \rho_{i} \mathbf{D}(\mathbf{u})$ for the $i$-th transport equation. For this choice of dispersion tensor, a complete mathematical theory was developed in [39] (see also [40]). However, this model was not widely accepted, and, currently, is not used in simulations.

Remark 3. In the literature of viscous fingering computations [4, 7], the assumption $\alpha_{L}=\alpha_{T}=0$ is often times made, and, consequently, the dispersion tensor $\mathbf{D}$ becomes isotropic. Although, in general, not representative of realistic scenarios in porous media flows, this assumption is the most common in many theoretical and analytical studies. Typically, anisotropic dispersion tends to stabilize flow and retard the development of viscous fingers.

For compressible flows or rocks, the dependency of the porosity and density on the pressure and the mass fractions is expressed as:

$$
\begin{aligned}
\phi(p, \mathbf{c}) & =\phi_{\mathrm{ref}} e^{C_{\phi}\left(p-p_{\mathrm{ref}}\right)+\mathbf{C}_{\mathrm{c}} \cdot \mathbf{c}}, \\
\rho_{i}(p, \mathbf{c}) & =\rho_{\mathrm{ref} ; i} e^{\boldsymbol{\beta}_{\rho ; i}\left(p-p_{\mathrm{ref}}\right)+\beta_{\mathrm{c}, i} \cdot \mathbf{c}} \approx \rho_{\mathrm{ref} ; i}\left(1+\boldsymbol{\beta}_{\rho ; i}\left(p-p_{\mathrm{ref}}\right)+\boldsymbol{\beta}_{\mathbf{c}, i} \cdot \mathbf{c}\right),
\end{aligned}
$$

where $\mathbf{c}=\left\{c_{1}, c_{2}, \ldots, c_{N_{c}}\right\} . C_{\phi}, \boldsymbol{\beta}_{\rho ; i}$ and $\mathbf{C}_{\mathbf{c}}$ are compressibility coefficients, $\boldsymbol{\beta}_{\mathbf{c} ; i}$ are volumetric solutal expansion coefficients and $\phi_{\text {ref }}$ and $\rho_{\text {ref }}$ are the values of porosity and density at the reference pressure state $p_{\text {ref }}$. Clearly, if the compressibility coefficients are zero, the incompressible limit is recovered. These coefficients are in general assumed 
constant, unless non-isothermal flows are considered. Summing equations (1) over $i$ and recalling (3) it is possible to obtain the mass conservation equation

$$
\partial_{t}(\phi \rho)+\nabla \cdot(\rho \mathbf{u})=q
$$

Remark 4. The porosity $\phi$ is frequently determined by solving the ordinary differential equation

$$
\frac{\partial \phi}{\partial p}=(1-\phi) \alpha_{p}
$$

where $\alpha_{p}$ is the coefficient of compressibility of the porous medium due to fluid pressure variations, related to the rock compressibility [33].

Remark 5. The employed porosity-pressure relation (6) is a simplified model. A more rigorous approach should relate the change in porosity to the volumetric strain of the rock, which in turn, is solved as the equilibrium solution to the force balance equation [41].

The velocity $\mathbf{u}$ at which the flow displaces is due to pressure gradients and gravitational forces, according to Darcy's law:

$$
\mathbf{u}=-\frac{\mathbf{K}}{\mu}(\nabla p-\rho \mathbf{g}) .
$$

The symbol $\mathbf{K}$ indicates the symmetric, positive-definite permeability tensor. The scalar $\mu$ indicates the viscosity of the solution, assumed to depend nonlinearly on the mass fractions, that is $\mu=\mu\left(c_{1}, c_{2}, \ldots, c_{N_{c}-1}\right)$. Substituting (10) into (1) and (8) it is possible to derive a closed system of equations in terms of the pressure $p$ and $N_{c}-1$ of the solution's components (the remaining component can be obtained using the constraint $\sum_{i}^{N_{c}} c_{i}=1$ ). In particular, we will consider the system of the first $N_{c}-1$ transport equations (1), together with their sum (8). Namely:

$$
\begin{aligned}
\partial_{t}\left(\phi \rho c_{i}\right)+\nabla \cdot\left(\rho \mathbf{u} c_{i}-\phi \rho \mathbf{D}(\mathbf{u}) \nabla c_{i}\right) & =q_{i}, \\
\partial_{t}(\phi \rho)+\nabla \cdot(\rho \mathbf{u}) & =q,
\end{aligned}
$$

with $i=1,2, \ldots, N_{c}-1$. This strategy is very effective in outlining the appropriate set of boundary conditions. In order to simplify the notation for equations $111-(12)$, let us introduce the mobility

$$
\boldsymbol{\kappa}:=\mathbf{K} / \mu \text {, }
$$

and the density-weighted quantities

$$
\begin{aligned}
& \tilde{\phi}:=\rho \phi, \\
& \tilde{\mathbf{u}}:=\rho \mathbf{u}, \\
& \tilde{\boldsymbol{\kappa}}:=\rho \boldsymbol{\kappa} . \\
& \tilde{\mathbf{g}}:=\rho \mathbf{g} .
\end{aligned}
$$

In practice, we can think of $(\tilde{\cdot})$ as the operator that multiplies the argument by $\rho$. With this definitions, (11) - (12) become

$$
\begin{aligned}
\partial_{t}\left(\tilde{\phi} c_{i}\right)+\nabla \cdot\left(\tilde{\mathbf{u}} c_{i}-\tilde{\phi} \mathbf{D} \nabla c_{i}\right) & =q_{i}, \\
\partial_{t} \tilde{\phi}+\nabla \cdot \tilde{\mathbf{u}} & =q .
\end{aligned}
$$

Analogously, 10] becomes

$$
\tilde{\mathbf{u}}=-\tilde{\boldsymbol{K}}(\nabla p-\tilde{\mathbf{g}})
$$


Observe that, for a given vector of mass fractions c, equation 19 is a parabolic equation for the pressure, since, substituting [20) into (19), yields

$$
\partial_{t} \tilde{\phi}(p)-\nabla \cdot(\tilde{\boldsymbol{\kappa}}(\nabla p-\tilde{\mathbf{g}}))=q .
$$

The coupling with the transport equations (18) occurs through the dependence of $\mu, \phi$ and $\rho$ on the mass fractions $c_{i}$. Typical boundary conditions associated with $(19)$ are Dirichlet boundary conditions on the pressure, and Neumann (flux) boundary conditions on the total flow flux $\tilde{\mathbf{u}}$. Let us decompose the boundary $\Gamma$ as follows: $\Gamma=\overline{\Gamma_{p ; D} \cup \Gamma_{p ; N}}$, with $\Gamma_{p ; D} \bigcap \Gamma_{p ; N}=\emptyset$. Dirichlet boundary conditions are enforced as

$$
p=p_{D}(\mathbf{x}, t), \quad \text { on } \Gamma_{p ; D},
$$

and Neumann boundary conditions are imposed as

$$
\tilde{\mathbf{u}} \cdot \mathbf{n}=\tilde{h}_{N}(\mathbf{x}, t), \quad \text { on } \Gamma_{p ; N} .
$$

Initial conditions on the pressure are imposed as

$$
p(\mathbf{x}, t=0)=p_{0}(\mathbf{x}), \quad \text { on } \Omega .
$$

Consider now a decomposition of the boundary $\partial \Omega=\Gamma=\overline{\Gamma_{c_{i} ; D} \cup \Gamma_{c_{i} ; N}}$ with $\Gamma_{c_{i} ; D} \bigcap \Gamma_{c_{i} ; N}=\emptyset$. Dirichlet boundary conditions are imposed at the Dirichlet boundary as

$$
c_{i}=c_{i ; D}(\mathbf{x}, t), \quad \text { on } \Gamma_{c_{i} ; D},
$$

and Neumann (flux) boundary conditions are imposed over $\Gamma_{c_{i} ; N}$, which is further subdivided into an inflow $\Gamma_{c_{i} ; N}^{-}$and an outflow $\Gamma_{c_{i} ; N}^{+}$as follows

$$
\begin{aligned}
& \Gamma_{c_{i} ; N}^{-}=\left\{\mathbf{x} \in \Gamma_{c_{i} ; N}: \tilde{\mathbf{u}}(\mathbf{x}) \cdot \mathbf{n}(\mathbf{x})<0\right\}, \\
& \Gamma_{c_{i} ; N}^{+}=\Gamma_{c_{i} ; N} \backslash \Gamma_{c_{i} ; N}^{-} .
\end{aligned}
$$

Specifically, we enforce inflow total flux boundary conditions as

$$
\left(\tilde{\mathbf{u}} c_{i}-\tilde{\phi} \mathbf{D} \nabla c_{i}\right) \cdot \mathbf{n}=h_{c_{i}}(\mathbf{x}, t), \quad \text { on } \Gamma_{c_{i} ; N}^{-},
$$

and free outflow boundary conditions as

$$
\left(\tilde{\mathbf{u}} c_{i}-\tilde{\phi} \mathbf{D} \nabla c_{i}\right) \cdot \mathbf{n}=c_{i} \tilde{h}_{N}(\mathbf{x}, t), \quad \text { on } \Gamma_{c_{i} ; N}^{+},
$$

As seen in (28), the total flux (the sum of the advective and diffusive fluxes) is enforced at the inflow. However, it is not possible, in general, to enforce the outflow flux for a specific component, which might not be present near the boundary itself. Condition (29) expresses the fact that the components present at an outflow boundary are free to exit the domain, with the appropriate relative concentrations. For the Neumann boundary conditions 28 - 29] to be well posed, the following compatibility conditions must also hold:

$$
\begin{gathered}
\Gamma_{p ; N}=\bigcup_{i} \Gamma_{c_{i} ; N}, \\
\tilde{h}_{N}(\mathbf{x}, t)=\sum_{i} h_{c_{i}}(\mathbf{x}, t),
\end{gathered}
$$

that is, the union of the inflow/outflow boundaries for the various component transport equations must coincide with the Neumann boundary for equation $\left[19\right.$, and the sum of inflow Neumann fluxes $h_{c_{i}}$ at a certain point on $\Gamma_{p ; N}^{-}$must coincide with the Neumann flux associated with the average flux $\tilde{h}_{N}$ of the solution. Clearly, the compatibility conditions are a direct consequence of the fact that the sum of (18) over the components $i$ yields (19). To complete the problem setting, initial conditions on the mass fraction distribution can be imposed as

$$
c_{i}(\mathbf{x}, t=0)=c_{i ; 0}(\mathbf{x}), \quad \text { on } \Omega .
$$


Remark 6. Another form of equation (12) is

$$
\rho S_{0}^{p} \frac{\partial p}{\partial t}+\nabla \cdot(\rho \mathbf{u})=q-\rho \phi \omega_{\mathbf{c}} \cdot \frac{\partial \mathbf{c}}{\partial t},
$$

where $S_{0}^{p}=\frac{\partial \phi}{\partial p}+\frac{\phi}{\rho} \frac{\partial \rho}{\partial p}$ represents the specific storativity of the porous medium with respect to pressure and $\omega_{\mathbf{c}, i}=$ $\frac{1}{\phi} \frac{\partial \phi}{\partial c_{i}}+\frac{1}{\rho} \frac{\partial \rho}{\partial c_{i}}$ is the sum of the coefficient of expansivity resulting from the change of the mass concentration of solute $i$ at constant pressure and the coefficient of compressibility of the fluid resulting from the change of the fluid pressure at constant mass fraction of the solute. Equation (33) is a convenient starting point for the Boussinesq approximation, derived by neglecting density variations within the mass balance equation 33. Density variations are kept only in the buoyancy term of the Darcy equation 10 . Accordingly, the equation of mass conservation takes the form

$$
S_{0}^{p} \frac{\partial p}{\partial t}+\nabla \cdot \mathbf{u}=\frac{q}{\rho}
$$

For the reactive transport in a rigid porous medium, this simplification reduces the mass conservation to the fluid volume conservation. For the rigorous justification of the Boussinesq approximation we refer to [42].

Next we follow [36] and write system [11)- 12 in the form

$$
\begin{aligned}
& \rho\left(\partial_{t}\left(\phi c_{i}\right)+\mathbf{u} \cdot \nabla c_{i}\right)=\nabla \cdot\left(\phi \rho \mathbf{D} \nabla c_{i}\right)+q_{i}-c_{i} q, \quad i=1, \ldots, N_{c}-1, \\
& D_{\phi} \rho=\partial_{t}(\phi \rho)+\mathbf{u} \cdot \nabla \rho=q-\rho \nabla \cdot \mathbf{u} .
\end{aligned}
$$

Applying the operator $D_{\phi}$ to 4 yields

$$
D_{\phi} \rho \sum_{i=1}^{N_{c}} \frac{c_{i}}{\rho_{i}}+\rho \sum_{i=1}^{N_{c}} \frac{1}{\rho_{i}} D_{\phi} c_{i}=0 .
$$

After using equations 35a- 35b in 35c, we obtain

$$
\nabla \cdot \mathbf{u}=\frac{q}{\rho}+\rho \sum_{i=1}^{N_{c}} D_{\phi} \frac{c_{i}}{\rho_{i}}
$$

\subsubsection{The incompressible limit}

The structure of the equations is in general degenerate parabolic. In the case in which the porosity and the species densities do not depend on the mass fractions nor on the pressure (e.g., the isobaric case), the equations can be further simplified by dividing each equation of type (1) by the corresponding density $\rho_{i}$ and summing up. After inserting 35a into 35d

$$
\nabla \cdot \mathbf{u}=\sum_{i=1}^{N_{c}} \frac{1}{\rho_{i}} \nabla \cdot\left(\phi \rho \mathbf{D} \nabla c_{i}\right)+\sum_{i=1}^{N_{c}} \frac{q_{i}}{\rho_{i}}=\nabla \cdot\left(\phi \rho \mathbf{D} \nabla\left(\frac{1}{\rho}\right)\right)+\sum_{i=1}^{N_{c}} \frac{q_{i}}{\rho_{i}},
$$

or, using Darcy's law 20,

$$
-\nabla \cdot(\boldsymbol{\kappa}(\nabla p-\rho \mathbf{g}))=\nabla \cdot\left(\phi \rho \mathbf{D} \nabla\left(\frac{1}{\rho}\right)\right)+\sum_{i=1}^{N_{c}} \frac{q_{i}}{\rho_{i}} .
$$

Therefore, it is sufficient to solve the pressure equation (35f) coupled with equation (1). In the incompressible case, the simplified notations (14)-(17) do not provide a substantial simplification to the equations, and are not used.

The initial and boundary conditions in the case of incompressible flow are very similar the compressible case. One important difference, however, is that there is no need for a pressure initial condition, as the pressure plays the role of a Lagrange multiplier enforcing the incompressibility condition 36 . 
Remark 7. The authors in [36] point out that the proposed equations can produce instabilities if a perturbation in permeability is applied, due to the presence of the right hand side of (35f). In contrast, the standard Boussinesq approximation, can only produce instabilities if perturbative mass fraction gradients are introduced, because in that case the right hand side of (35f) vanishes.

Remark 8. In the limit of small dispersion, that is when $\mathbf{D}$ becomes negligible, (8) can be further simplified to

$$
\nabla \cdot \mathbf{u}=\sum_{i=1}^{N_{c}} \frac{q}{\rho_{i}} .
$$

\subsection{Modeling single phase mixtures through upscaling}

The multi-component mixture modes presented so far are derived in the engineering literature by thermodynamically consistent direct modeling. On the one hand, this approach leaves a great liberty for the constitutive relations. On the other hand, there exists a well developed mixture theory in continuum mechanics, based on the first principles of rational mechanics, and valid at the pore scale level. In this section we will present a model for a two-component mixture, obtained by upscaling a continuum mechanics first-principle pore scale model. We start with some notation:

$\mathbf{v}$ is the mean velocity of the two-component single phase incompressible mixture;

$c_{1}$ represents the solvent mass fraction;

$p$ is the modified mixture pressure;

$\rho_{1}, \rho_{2}$ are constant proper mass densities of the components, with $\gamma=\rho_{1}-\rho_{2}>0$, so that $\rho\left(c_{1}\right)=\gamma c_{1}+\rho_{2}$ is the mixture density; 11

$\mu\left(c_{1}\right)$ is the mixture viscosity and $\lambda$ is the mixture diffusion coefficient.

The mixture flow and diffusion in the pore space of a rigid porous medium are governed by the system of equations

$$
\begin{aligned}
\rho\left(c_{1}\right)\left(\partial_{t} \mathbf{v}+(\mathbf{v} \nabla) \mathbf{v}\right) & =-\nabla p+\nabla \cdot\left(\mu\left(c_{1}\right)\left(\nabla \mathbf{v}+(\nabla \mathbf{v})^{T}\right)\right)+\gamma \lambda\left((\nabla \mathbf{v}) \nabla c_{1}+\left(\nabla \nabla c_{1}\right) \mathbf{v}\right)+\rho\left(c_{1}\right) \mathbf{f}, \\
\nabla \cdot \mathbf{v} & =0, \\
\mathbf{v} & =0 \quad \text { on pore boundaries, } \\
\partial_{t} c_{1}+\mathbf{v} \cdot \nabla c_{1} & =\lambda \Delta c_{1}, \\
\mathbf{n} \cdot \nabla c_{1} & =0 \quad \text { on pore boundaries, }
\end{aligned}
$$

whose mathematical study is presented in detail in [43] and references therein. We now distinguish between the two characteristic scales of problem (37a)-(37e): The (microscopic) pore scale and the (macroscopic) domain scale, and we define their ratio as $\varepsilon$, a small parameter. For simplicity, we limit our considerations to the case when the global Péclet and Reynolds numbers are of order 1. This implies that the corresponding pore-level characteristic numbers are small and the nonlinear transport effect will not play an important role, allowing separation between the macroscopic (slow) and microscopic (fast) scales.

The porous medium in problem $37 \mathrm{a}$ - $-37 \mathrm{e}$ is considered as statistically homogeneous, and in this case, a simple geometric assumption is periodicity at the microscopic level, that is, after rescaling, we consider a microscopic $\varepsilon$-periodic open subset $\Omega_{\varepsilon}$ of $\mathbb{R}^{n_{d}}, n=2,3$, while the macroscopic domain $\Omega$ is smooth and bounded. The unit periodicity cell associated with $\Omega_{\varepsilon}$ is $Y=(0,1)^{n_{d}}$, on which we consider a smooth partition $S^{0} \cup Y^{0}$, where $S^{0}$ is the solid part and $Y^{0}$ is the fluid part (its periodic extension is a smooth connected open subset, see Fig. 1). By periodic translations we obtain $Y_{\varepsilon}^{j}=\varepsilon\left(Y^{0}+j\right), S_{\varepsilon}^{j}=\varepsilon\left(S^{0}+j\right), B_{\varepsilon}^{j}=\varepsilon\left(\partial S^{0}+j\right), A_{\varepsilon}=\bigcup_{j \in \mathbb{Z}^{n} d} Y_{\varepsilon}^{j}$ and $B_{\varepsilon}=\partial \Omega_{\varepsilon}$, where $A_{\varepsilon}$ is connected and its boundary $B_{\varepsilon}$ is smooth. $\Omega_{\varepsilon}$ is the intersection of $A_{\varepsilon}$ with $\Omega$, with the exclusion of all solid parts of the periodic cell that intersect $\partial \Omega$. We suppose that system $37 \mathrm{a}-37 \mathrm{e}$ is in dimensionless form with $\mu, \gamma, \lambda$ are $O(1)$

\footnotetext{
${ }^{1}$ Note that for small $\gamma c_{1}, 1 / \rho=1 /\left(\rho_{2}+\gamma c_{1}\right) \approx \chi / \rho_{1}+(1-\chi) / \rho_{2}$, with $\chi=c_{1} \rho_{1} / \rho_{2}$
} 


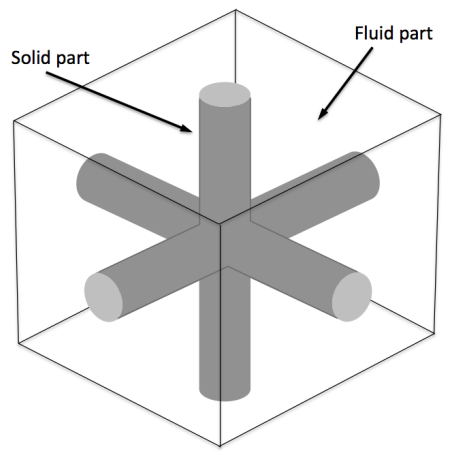

Figure 1: The unit pore $Y$ with the solid part $S^{0}$ (gray) and its complement $Y^{0}=Y \backslash S^{0}$, the fluid part.

and $f=\varepsilon^{-2} f^{e f f}$. Then the effective behavior of the solution of 37a $-37 \mathrm{e}$ is studied using the two-scale expansion

$$
\begin{aligned}
\mathbf{v} & \approx \frac{1}{\mu\left(s^{e f f}\right)} \sum_{j=1}^{n_{d}} \mathbf{w}^{j}\left(\frac{x}{\varepsilon}\right)\left(\rho\left(s^{e f f}\right) f_{j}-\frac{\partial p^{e f f}}{\partial x_{j}}\right)+O(\varepsilon), \\
c_{1} & \approx s^{e f f}+\varepsilon \sum_{j=1}^{n_{d}} q^{j}\left(\frac{x}{\varepsilon}\right) \frac{\partial s^{e f f}}{\partial x_{j}}+O\left(\varepsilon^{2}\right), \\
\varepsilon^{2} p & \approx p^{e f f}(x, t)+O(\varepsilon) .
\end{aligned}
$$

The oscillating functions $\mathbf{w}^{j}$ and $q^{j}$ can be calculated inserting the above expansions into 37a - 37e. Hence, the cell problem for the $Y$-periodic functions $\mathbf{w}^{j}, j=1, \ldots, n_{d}$, is

$$
\begin{aligned}
& -\Delta_{y} \mathbf{w}^{j}(y)+\nabla_{y} \pi^{j}(y)=\mathbf{e}^{j} \quad \text { in } Y^{0}, \\
& \operatorname{div}_{y} \mathbf{w}^{j}(y)=0 \quad \text { in } Y^{0}, \\
& \mathbf{w}^{j}(y)=0 \quad \text { on } \partial S^{0} \text {. }
\end{aligned}
$$

The dimensionless permeability tensor $\mathbf{K}^{0}$ is then given by

$$
\mathrm{K}_{i j}^{0}=\int_{Y^{0}} w_{i}^{j}(y) d y .
$$

where $\mathbf{K}^{0}$ is a symmetric positive definite tensor and $\mathbf{K}=\varepsilon^{2} \mathbf{K}^{0}$ corresponds to the physical permeability. Analogously, the $Y$-periodic functions $q^{j}, j=1, \ldots, n_{d}$, are the solutions to

$$
\begin{aligned}
& \Delta_{y} q^{j}=0 \quad \text { in } Y^{0} \text {, } \\
& \frac{\partial q^{j}}{\partial \mathbf{n}}=-n_{j} \quad \text { on } \partial S^{0} \text {. }
\end{aligned}
$$

The effective diffusion tensor $\mathbf{D}$ is then given by

$$
\mathbf{D}=D_{i j}=\lambda D_{i j}^{0}=\lambda\left(\phi \delta_{i j}+\int_{Y^{0}} \frac{\partial q^{i}}{\partial y^{j}} d y\right),
$$

where $\mathbf{D}$ is a symmetric positive definite tensor. The Darcy's velocity $\mathbf{u}$ is given by

$$
\mathbf{u}=\frac{K}{\mu\left(s^{e f f}\right)}\left(\rho\left(s^{e f f}\right) \mathbf{f}-\nabla p^{e f f}\right),
$$


and, in summary, the effective equations are

$$
\begin{aligned}
\nabla \cdot\left(\frac{\mathbf{K}}{\mu\left(s^{e f f}\right)}\left(\rho\left(s^{e f f}\right) \mathbf{f}-\nabla p^{e f f}\right)\right) & =0, \\
\phi \frac{\partial s^{e f f}}{\partial t}+\mathbf{u} \cdot \nabla s^{e f f}-\nabla \cdot\left(\mathbf{D} \nabla s^{e f f}\right) & =0 .
\end{aligned}
$$

The system $(42 b)-(42 c)$ represents the Boussinesq approximation of $35 \mathrm{ab}-(35 \mathrm{f})$ and, due to the coupling between the flow and convective transport, instabilities may by induced by the dependency of the viscosity and density on the concentration.

For a detailed homogenization analysis, including the proof of convergence of the pore scale quantities to the upscaled quantities, we refer to [44]. The simplified case in which all miscible components have equal densities was considered in [45]. In [44], the existence and uniqueness of a smooth solution of the system (42b)-(42c) was established under the assumption of small viscosity variations. In [3], existence of a weak solution was proved for the system of equations (42b)- (42c) in the case of a velocity-dependent dispersion tensor [37]. Existence and uniqueness of a smooth solution were proved in [46] and [47], under the condition of small viscosity variations. These rigorous mathematical results were confirmed by numerical simulations.

We recall once more that $42 \mathrm{~b}-42 \mathrm{c}$ ) were obtained under a restriction on the magnitude of the Péclet and Reynolds numbers, and that its importance resides in the validation of the models used in practical simulations. For fast flows, Darcy law (42a) ceases to be valid, and the homogenization of the momentum equation 37a for large global Reynolds numbers leads to a two-pressures Navier-Stokes system with the coexistence of both slow and fast scales (see [48], [49], [50] and [51] and references therein). In general, fast and slow scales cannot be separated. Nevertheless, it was rigorously proved in [50] that the solutions to the slow/fast scale two-pressures Navier-Stokes homogenized problem can be well approximated by a cubic Forchheimer filtration law, dependent only on macroscopic unknowns. For large Péclet numbers, expression 441 would change and the tensor $\mathbf{D}$ would depend on the velocity field. Again, the separation of the scales is not possible and in Section 2.4 we will further discuss dispersion effects linked to high Péclet numbers.

An alternative derivation of the model for miscible flows in porous media can be obtained using the heterogeneous multiscale method (HMM) for numerical homogenization [52]. The heterogeneous multiscale method was used for the numerical homogenization of the two-phase immiscible flows from Darcy's level to the reservoir level (see [53, 54, 55] and references therein), but not for the upscaling from the pore level. In principle, the general strategy of the HMM numerical homogenization could be used, jointly with the rigorous homogenization results presented in this section, to handle more complicate chemistry and non-periodic porous media. We also note that the authors of [55] study the immiscible two-phase flows with only one single component, and the dispersion is not treated. Similarly, in [53] the effect of capillary pressure was neglected. Therefore, in our assessment, the HMM approach to miscible flows is a promising approach that may require further developments.

A related approach is to use multiscale finite element methods, which, in the related literature by Efendiev, Hou and collaborators, are also referred to as the multiscale finite volume method (MsFVEM). A detailed presentation of the MsFVEM framework and its applications to two-phase immiscible and miscible flow is found in [56, 57, 58] and references therein. In these works, most of the attention is brought to the numerical homogenization of the immiscible two-phase flows from Darcy to the reservoir level. The author of [59] presents a detailed application to miscible flows with heterogeneities at the Darcy level, but neglecting capillary pressures and pore scale dispersion, so that an effective dispersion was derived from the closure relations developed in [60, 61].

\subsection{Linear stability analysis}

The stability of miscible flows through porous media has been studied using the classical methods of hydrodynamic stability theory, which we briefly summarize. For the physical introduction to the flow stability with extensive examples, we refer to Drazin [62] and Drazin and Reid [63]. For the mathematical theory, the classical reference is Yudovich [64]. First, many problems of applied interest are written in the evolution form

$$
\frac{d \mathbf{b}}{d t}=\mathbf{F}(t, \alpha, \mathbf{b}), \quad \mathbf{b}(0)=\mathbf{b}_{0},
$$


where $t \geq 0$ is the time variable, $\alpha$ is a real parameter, $\mathbf{b}$ is the unknown vector-valued function and $\mathbf{F}$ is a given nonlinear operator (an example is system (11)-(12)). A stationary solution $\mathbf{w}$ for (43) is obtained solving

$$
\mathbf{F}(t, \alpha, \mathbf{w})=0 \text {. }
$$

For a time-periodic nonlinear operator $\mathbf{F}$, time-periodic solutions are possible. Once a stationary solution $\mathbf{w}$ is computed, 43] is then reduced to a local form for the unknown $\mathbf{a}=\mathbf{b}-\mathbf{w}$, namely,

$$
\frac{d \mathbf{a}}{d t}=\mathbf{F}(t, \alpha, \mathbf{a}+\mathbf{w}), \quad \mathbf{a}(0)=\mathbf{a}_{0}=\mathbf{b}_{0}-\mathbf{w},
$$

which is called the nonlinear perturbation equation and is assumed to have a unique solution, at least for a set of initial values dense in some neighborhood of zero. The solution can also be interpreted as a nonlinear mapping parametrized over time and acting on the initial condition, that is $\mathbf{a}(t)=\mathcal{U}(t) \mathbf{a}_{0}$.

Many definitions of solution stability with respect to the initial data are known. At a minimum, stability must require that: 1) for "small" $\mathbf{a}_{0}$, the Cauchy problem (45) has a solution for all $t>0$; and 2) the mapping $\mathcal{U}$ is continuous. We consider next the definition of stability introduced in [64]):

Let $X_{0}$ and $X_{1}$ be Banach spaces. The fixed point 0 of the one-parameter family of mappings $\mathcal{U}(t)$ is called ' $\eta$-stable $\left(X_{0}, X_{1}\right)$ ' if

1. for any $\mathbf{d} \in X_{0}$ in some neighborhood of $\mathbf{a}_{0},\|\mathbf{d}\|_{X_{0}}<\delta_{0}$, the vector $\mathcal{U}(t) \mathbf{d} \in X_{1}$ for any $t>0$;

2. for any $\eta, \varepsilon>0$ there exists $\delta>0$ such that $\|\mathbf{d}\|_{X_{0}}<\delta$ implies that $\|\mathcal{U}(t) \mathbf{d}\|_{X_{1}}<\varepsilon$ for $t>\eta$.

In addition, if $\|\mathcal{U}(t) \mathbf{d}\|_{X_{1}} \rightarrow 0$ as $t \rightarrow+\infty$ is verified, then asymptotic stability $\left(X_{0}, X_{1}\right)$ holds. In particular, the stationary solution $\mathbf{w}$ of $(44)$ is called asymptotically stable if $\mathbf{w} \rightarrow \mathbf{0}$, as $t \rightarrow+\infty$.

The study of linear stability requires the Frechet derivative $\nabla_{\mathbf{b}} \mathbf{F}$ of $\mathbf{F}$, since the behavior of the perturbation $\mathbf{w}+\delta \mathbf{z}$ of $\mathbf{w}$ is governed by a linearized problem for $\mathbf{z}$, given by

$$
\frac{d \mathbf{z}}{d t}=\nabla_{\mathbf{b}} \mathbf{F}(t, \alpha, \mathbf{b}) \mathbf{z} .
$$

In general, linear stability does not imply (nonlinear) stability, but may provide often times an insight (e.g., when the problem has a Liapounov functional).

A very frequently studied case is the one in which instabilities develop from a stationary base flow. In this particular situation the velocity is independent of $t$, and, as a consequence, the linearized problem has coefficients also independent of $t$.

If problem (46) falls within the classical parabolic theory and separation of the variables applies, then the general solution of the initial-value problem is a linear superposition of normal modes, in the form of products of eigenfunctions and exponentials of the type $\exp \{\sigma t\}$, where $\sigma$ is an eigenvalue. If the flow domain is bounded, then the eigenvalues $\sigma$ 's form a set that is discrete and countable, and occur as either real or in complex conjugate pairs. Then, denoting by $\mathfrak{R}(\zeta)$ the real part of $\zeta \in \mathbb{C}$, the base flow solution of 44 is stable if $\mathfrak{R}(\sigma)<0$ for all the eigenvalues $\sigma$ and unstable if $\mathfrak{R}(\sigma)>0$ for at least one eigenvalue $\sigma$, because the modes have exponential growth. In the case of $\mathfrak{R}(\sigma)=0$, that is of neutral stability, the linear theory does not allow to make any conclusions on stability.

If, in addition, the base flow is invariant under translation or rotation, then one or more of the space variables can be separated. For example, stability analyses are often performed for base flows that are independent of $x$ and for which the perturbations take the form $\mathbf{u}(y, z) \exp (i k x+\sigma t)$.

A classic reference for earlier studies on stability of flows thorough porous media is the review article by Homsy [9], in which the influence of viscosity and gravity on fingering phenomena is discussed in detail for miscible and immiscible flows. Although in [9] both immiscible two-phase flows and incompressible miscible flows were considered, in what follows, we limit our attention to the latter. In the literature (see [9] and reference therein), the following particular case of miscible displacement is often considered:

$$
\begin{aligned}
\mathbf{u} & =\frac{\mathrm{K}}{\mu(c)}(-\nabla p+\rho \mathbf{g}), \\
\nabla \cdot \mathbf{u} & =0, \\
\frac{D c}{D t} & =\frac{\partial c}{\partial t}+\mathbf{u} \cdot \nabla c=\nabla \cdot(\mathbf{D} \nabla c),
\end{aligned}
$$


where $c$ is the concentration, scaled between 0 and 1 , and $\mathrm{K}$ is the scalar permeability coefficient, that is $\boldsymbol{\kappa}=\mathrm{K} / \mu \mathbf{I}$. Hence 47a)-(47c) represents a slight modification to 42b)-(42c), and the existence of a smooth stationary solution $\left\{p_{0}, \mathbf{u}_{0}, c_{0}\right\}$ was studied in [46]. If, for simplicity, we suppose that the dispersion tensor $\mathbf{D}$ is independent of $\mathbf{u}$ and $\mathbf{g}=\mathbf{0}$, then the linearized problem about $\left\{p_{0}, \mathbf{u}_{0}, c_{0}\right\}$ reads

$$
\begin{aligned}
\frac{\partial(\delta c)}{\partial t}+\mathbf{u}_{0} \cdot \nabla(\delta c) & =\nabla \cdot\left(\mathbf{D} \nabla(\delta c)-c_{0} \delta \mathbf{u}\right), \\
\nabla \cdot \delta \mathbf{u} & =0, \\
\delta \mathbf{u} & =-\frac{K}{\mu\left(c_{0}\right)} \nabla p-\mu^{\prime}\left(c_{0}\right) \mathbf{u}_{0} c,
\end{aligned}
$$

where $\{\delta p, \delta \mathbf{u}, \delta c\}$ is the perturbation to the base stationary solution $\left\{p_{0}, \mathbf{u}_{0}, c_{0}\right\}$. For smooth $\left\{p_{0}, \mathbf{u}_{0}, c_{0}\right\}$ the mapping $c \rightarrow\{p, \mathbf{u}\}$ is compact and the corresponding spectral problem can be studied using a variational variant of the classical Riesz-Schauder theory (see e.g. [65]), although symmetry would be lost. We note that, contrary to the case of gravitational instabilities discussed in Section 2.5, we are not aware of any rigorous mathematical study of problem (48a)-(48c) and corresponding spectral problem.

The discussion presented in [9] relies on the dimensionless numbers

$$
\begin{aligned}
& \mathrm{Pe}=\frac{U L}{D_{0}} \text { (Péclet number), } \quad M=\frac{\mu_{1}}{\mu_{2}} \text { (mobility ratio), } \\
& A=\frac{\mu_{1}-\mu_{2}}{\mu_{1}+\mu_{2}} \text { (viscosity contrast), } \quad G=\frac{\left|\rho_{1}-\rho_{2}\right| g K}{\left(\mu_{1}+\mu_{2}\right) U} \text { (gravity group), }
\end{aligned}
$$

where $U$ is the characteristic velocity and $D_{0}$ the reference value of the dispersion coefficient.

Most often, the stability of a specific time-dependent mobility profile is studied. An example is the stability of a rectilinear flow for a discontinuous distribution of viscosity in a coordinate system moving with the injection velocity [4], for which $\mathbf{u}_{0}=\mathbf{0}$ and $c_{0}=\frac{1}{2} \operatorname{erfc}(x /(2 \sqrt{t}))$. In that analysis, perturbations are assumed to grow or decay faster than the rate of evolution of the base solution, which is frozen at time $t_{0}$ and treated as steady. Then the perturbation $\{\delta \mathbf{u}, \delta c\}=\left\{\delta \mathbf{u}_{0}(x), \delta c_{0}(x)\right\} \exp \left(\sigma\left(t_{0}\right) t+i k y\right)$ is studied. Related work is in [66], where a local stability criterion was established, and further references are [67, 68]. The stability of radial miscible flows was studied in several references among which we mention [69, 70, 7, 71].

In [9], it was recognized that this parametrization is incomplete for a full description of realistic porous materials, and hope was placed in the use of simulations to better understand the pore scale fluid mechanics. The authors of $[14,15,72,73,74,75,6]$ studied the evolution of two-dimensional miscible porous media flows in the quarter fivespot problem described in Section 3.2 In [76], the relative influence of molecular diffusion and velocity-induced dispersion were also accounted for in the linear stability analysis. It was shown that, in general, velocity-induced dispersion has a stabilizing effect on displacements with respect to the case of simple molecular diffusion. This is somewhat expected, since the dispersion coefficient in a realistic porous medium is typically assumed to grow with increasing velocity. In [46, 47] the nonlinear stability was rigorously proved for $G=0$ and $A$ small. In [77], bounds were derived for the growth of small perturbations in porous media displacements in the absence of diffusion and dispersion. The effects of both isotropic and highly anisotropic dispersion in porous media were treated in the linear stability analysis of [4].

In [9], the miscible Hele-Shaw model is proposed as a convenient analogue to porous media flows, and discussed in detail. The Hele-Shaw model is associated with creep flow through a (small) gap of size $b$ between two parallel planes with characteristic length $L$. Viscous fingering is caused by variations in viscosity, due to variations in solvent concentrations. Establishing linear stability is complicated not only because of the difficulties with the linearized problem, but also through the time dependent nature of boundary conditions, which typically produce non stationary base solutions. In [78, 79], focus was directed to the miscible flows in Hele-Shaw cells in cylindrical tubes and radial geometry.

In [9] it was observed that, in the case of zero dispersion and for a convected coordinate system, the solvent concentration is time independent, and a connection to the two-phase immiscible flows with zero surface tension was made. For normal modes proportional to $\exp \{\sigma t+i k y\}$ one finds the dispersion relation $\sigma=(A+G) k$. This result is 
non-physical and the dispersion effects have to be added to the perturbation, to obtain

$$
\sigma=\frac{1}{2}\left(A k-k^{3} \mathrm{Pe}^{-2}-k \sqrt{k^{2} \mathrm{Pe}^{-2}+2 A k \mathrm{Pe}^{-1}}\right) .
$$

This relation implies that a cutoff length of unstable modes is induced by the Péclet number, and that, for large Péclet numbers, the flow behavior may be very complex.

\subsection{Dispersion}

In miscible multi-component flows different phenomena coexist at different order of magnitude. It is fairly common that transport dominates diffusion and that gravitational effects occur at a different timescale with respect to convection/diffusion. We note that the Péclet number plays a fundamental role in the non-dimensional form of the equations, and that the simplest model for a porous medium is a bundle of parallel capillary tubes, which we describe next, in the context of Hele-Shaw cell, serving as a prototype for the complex geometries of porous media.

\subsubsection{Classical Taylor dispersion in tubes and Hele-Shaw cells}

Taylor's dispersion is one of the most studied transport mechanisms in miscible flow dispersion. The simplest setting consists in the injection of a solute into a slit channel (a two-dimensional Hele-Shaw cell). If gravitational effects are neglected and viscosity is assumed not to depend on the solute concentration, Poiseuille's flow is responsible for the transport of the solute. Three distinct regimes are observed: (a) diffusion-dominated mixing, (b) Taylor dispersion-mediated mixing and (c) chaotic advection.

In diffusion-dominated mixing, the velocity is small and Péclet number is of order one or smaller. Hence, molecular diffusion plays a dominant role and no solute dispersion is observed. This regime is well understood but rarely present in miscible displacement flows. If the flow rate is increased and the Péclet number becomes large, that is $\mathrm{Pe}>>1$, then the system is dominated by Taylor dispersion-mediated mixing. In the third regime, chaotic advection is spurred by turbulent mixing, for very high values of the Péclet number.

Returning to the case of Taylor dispersion, there exists a timescale at which transversal molecular diffusion smears the contact discontinuity into plug flow. In 1953 Taylor found an explicit formula for the effective long-time axial diffusivity, proportional to the square of the transversal Péclet number and occurring in addition to the molecular diffusivity. A vast literature was developed on the subject following the pioneering work of Taylor [80].

The most notable references are the article by Aris [81], where Taylor's intuitive approach was explained through a moment expansion, the lecture notes in [82], where a probabilistic justification is given for Taylor's dispersion, and the papers [83] and [84], which describe the development of the approach of center manifolds. This method allowed to calculate approximations at any order for the original Taylor's model but without a rigorous justification. A mathematically rigorous analysis with an error estimate for the Taylor dispersion formula (generalized to reactive flows) was obtained by Mikelić and coworkers in recent articles [85, 86, 87, 88, 89]. A rigorous analysis can be developed through anisotropic singular perturbation [85]. This strategy provides an error estimate for the approximation and, consequently, a mathematically rigorous justification of the effective (upscaled) models. The formal expansion is developed using the methodology introduced in [90], which we summarize below.

The small parameter of the problem is $\varepsilon=b / L$ (the ratio of the gap size and an observable characteristic length). We introduce the characteristic longitudinal time scale $T_{L}=L / U$ ( $U$ is the scaling for the flow velocity) and the characteristic transversal time scale $T_{T}=b^{2} / D_{0}$. As before, the longitudinal Péclet number is $\mathrm{Pe}=L U / D_{0}$. We set $\mathrm{Pe}=\varepsilon^{-\alpha}$. Then

$$
\frac{T_{T}}{T_{L}}=\frac{U b^{2}}{L D_{0}}=\frac{U L}{D_{0}}\left(\frac{b}{L}\right)^{2}=\varepsilon^{2} \mathrm{Pe}=O\left(\varepsilon^{2-\alpha}\right) .
$$

In Taylor's original work [80] the case $0 \leq \alpha<2$ is analyzed, and experimental measurements are presented for $\alpha=1.6$ and $\alpha=1.9$. We also introduce the transversal Péclet number $\mathrm{Pe}_{T}=b U / D_{0}=\varepsilon \mathrm{Pe}=\varepsilon^{1-\alpha}$, so that $T_{T} / T_{L}=\varepsilon \mathrm{Pe}_{T}$. 
The domain of interest is the semi-infinite strip $Z^{+}=\mathbb{R}_{+} \times(0,1)$. Using the antisymmetry of $c^{\varepsilon}=c$, the nondimensional form of the equations of motion with respect to the time scale $T_{L}$ yields

$$
\begin{aligned}
\frac{\partial c^{\varepsilon}}{\partial t}+\left(1-y^{2}\right) \frac{\partial c^{\varepsilon}}{\partial x}-\varepsilon^{\alpha} \partial_{x x} c^{\varepsilon}-\varepsilon^{\alpha-2} \partial_{y y} c^{\varepsilon}=0 & \text { in } Z^{+} \times(0, T) \\
-\left.\varepsilon^{\alpha-2} \frac{\partial c^{\varepsilon}}{\partial y}\right|_{y=1}=0 & \text { on } \mathbb{R}_{+} \times(0, T), \\
\partial_{y} c^{\varepsilon}(x, 0, t)=0, & (x, t) \in \mathbb{R}_{+} \times(0, T) .
\end{aligned}
$$

An effective equation of motion can be obtained by studying the behavior as $\varepsilon \rightarrow 0$ of a solution of (60a) - 60d with square integrable gradients in $x$ and $y$. This can be achieved by means of a two-scale expansion with respect to the time-scale ratio $T_{T} / T_{L}=\varepsilon^{2-\alpha}$.

$$
c^{\varepsilon}=c_{F}^{0}(x, t ; \varepsilon)+\varepsilon^{2-\alpha} c_{F}^{1}(x, y, t)+\varepsilon^{2(2-\alpha)} c_{F}^{2}(x, y, t)+\ldots
$$

leading to

$$
0=\varepsilon^{\alpha-2} \partial_{y y} c_{F}^{0}+\varepsilon^{0}\left(\partial_{y y} c_{F}^{1}-\frac{\partial c_{F}^{0}}{\partial t}-\left(1-y^{2}\right) \frac{\partial c_{F}^{0}}{\partial x}\right)+\varepsilon^{2-\alpha}\left(\partial_{y y} c_{F}^{2}-\frac{\partial c_{F}^{1}}{\partial t}-\left(1-y^{2}\right) \frac{\partial c_{F}^{1}}{\partial x}\right)+\varepsilon^{\alpha}\left(\partial_{x x} c_{F}^{0}+\varepsilon^{2-\alpha} \partial_{x x} c_{F}^{1}\right)+\ldots
$$

The first term implies that $c_{F}^{0}$ is independent of $y$. The second term gives a PDE in $y$ for $c_{F}^{1}$. Then we use Fredholm's alternative to obtain the effective equations. 2 However, we point out that directly applying Fredholm's alternative gives a linear transport equation for $c_{F}^{0}$ that is not immediately useful for our model. An improved approximation can be obtained using the strategy of Rubinstein and Mauri [90] and embed the linear transport equation into the next order equations. Applying again Fredholm's alternative, effective equations containing Taylor's dispersion type terms are obtained, namely:

$$
\partial_{t} c_{F}^{0}+\frac{2}{3} \partial_{x} c_{F}^{0}=\left(1+\frac{8}{945} \varepsilon^{2} \mathrm{Pe}^{2}\right) \partial_{x x} c_{F}^{0} .
$$

Note that $2 / 3$ is the mean of the dimensionless Poiseuille velocity and the constant $8 / 945$ is the second order moment of the polynomial $P_{4}(y)=y^{2} / 6-y^{4} / 12-7 / 180$, yielding $c_{F}^{1}=P_{4}(y) \partial_{x} c_{F}^{0}$. In dimensional form, 54 reads

$$
\partial_{t} c^{T a y}+\frac{2 U}{3} \partial_{x} c^{T a y}=D_{0}\left(1+\frac{8}{945} \frac{b^{2} U^{2}}{D_{0}^{2}}\right) \partial_{x x} c^{T a y},
$$

which is the expression found by Taylor [80]. Note that the operator $D_{0} \Delta_{x, y}$ is upscaled to $D_{0}\left(1+\frac{8}{945} \frac{b^{2} U^{2}}{D_{0}^{2}}\right) \partial_{x x}$. For the error estimate (and, consequently, the rigorous justification of the Taylor dispersion formula) we quote Theorem 1 from [89]. After neglecting the chemical reactions, it yields

$$
\left\|c^{\varepsilon}-c_{F}^{0}-\varepsilon^{2-\alpha} P_{4}(y) \partial_{x} c_{F}^{0}\right\|_{L^{2}\left(Z^{+} \times(0, T)\right)} \leq C \varepsilon^{(2-\alpha)(3 / 2-\delta)}, \quad \forall \delta \in(0,1) .
$$

An $L^{\infty}$-bound in space holds as well, but would break down for some $\alpha<2$. Inspection of equation (54) shows that for $\alpha \approx 0$, there is no dispersion correction, for $\alpha \approx 1$ the Taylor correction is balanced with the molecular diffusion, and for $\alpha$ approaching 2 the molecular diffusion is negligible compared with the dispersion term. $\alpha=2$ is therefore a threshold value, beyond which we have regime (c) of turbulent mixing. When turbulent mixing occurs, the dependence on $\mathbf{P e}_{T}$ becomes either logarithmic, sub-linear or linear (see [91] and chapter 9 from [92]) and, in particular, the two-scale expansion (53) does not apply.

One important observation is that, when $\alpha$ approaches 2, the error estimate (56) experiences considerable deterioration. Then, since the dispersion term originates from transport, one can contest the validity of parabolic transport equations for the effective solute concentration. In fact, in the environmental and soil sciences, this type of modeling leads to some non-physical properties of the overall model:

\footnotetext{
${ }^{2}$ We recall, for a non-mathematical reader, that Fredholm's alternative gives a necessary and sufficient condition for the solvability of an equation, and it is especially useful in the critical situation when the spectrum of the operator is considered. The corresponding boundary value problem in the $y$-variable admits a solution if and only if the mean of the right hand side with respect to the transversal variable $y$ is equal to the value of the flux at $y=1$. We refer to the textbooks as [65], for Fredholm's theory for partial differential equations.
} 
(i) While frequently neglected in the equations from which the analysis begins, effective dispersion in the longitudinal direction is present in Taylor's dispersion model. The upscaled effective equation then predict an infinite speed of propagation of perturbations, which is not observed in experiments.

(ii) For purely convective flows, solute particles follow streamlines. Hence when flow reversal occurs, particles should return to their original position. Obviously, this is not true anymore in the presence of transversal diffusion, since, in this case, particles move randomly in the transverse direction between streamlines. On physical grounds one expects at least a variance decrease after flow reversal (partial reversibility).

These reasoning and observations motivated Scheidegger [93] to propose, already in 1958, the one-dimensional telegraph equation

$$
\frac{\partial^{2} c}{\partial t^{2}}+\frac{1}{\tau} \frac{\partial c}{\partial t}=\sigma_{v}^{2} \frac{\partial^{2} c}{\partial x^{2}}
$$

as a model for dispersion in porous media. Here, $\sigma_{v}$ is the velocity variance and $\tau$ a relaxation parameter. In the case of dispersion in a tube, Camacho [94, 95, 96] developed upscaled hyperbolic models, based on physical concepts from irreversible thermodynamics. His model equation reduces to the telegraph equation if molecular diffusion is neglected (see [97] for further developments of the model).

One of the potential drawbacks of telegraph equation models is the presence of an undetermined parameter. Motivated by this issue, Balakotaiah and coworkers [98, 99] proposed to use instead Liapounov-Schmidt reduction in combination with a perturbation argument, and derived the dispersion equation

$$
\frac{\partial c}{\partial t}+U \frac{\partial c}{\partial x}+\frac{U d^{2}}{48 D_{0}} \frac{\partial^{2} c}{\partial x \partial t}=D_{0} \frac{\partial^{2} c}{\partial x^{2}}
$$

When $D_{0}$ is very small, the right hand side could be neglected, corresponding to the hyperbolic limit. In this limit case, (58) becomes Goursat's equation and does not suffer from the problematic aspects described in (i) and has also the partial reversibility property mentioned in (ii).

The rigorous mathematical justification of the formal results and observations made by Balakotaiah and coauthors is due to Mikelić and van Duijn in [100]. The result from Theorem 2.2, [100], reads:

Let $2>\alpha>4 / 3$, let the initial and boundary conditions be smooth and compatible and the boundary condition be appropriately upscaled as well. Then for all $T \in(0,+\infty)$ we have

$$
\varepsilon^{\alpha-2}\left(c^{\varepsilon}-c^{e f f}\right)+\frac{3}{2} P_{4}(y) \frac{\partial c^{e f f}}{\partial t} \rightarrow 0 \quad \text { in } \quad L^{2}\left(Z^{+} \times(0, T)\right), \quad \text { as } \varepsilon \rightarrow 0
$$

where

$$
\left\{\begin{array}{l}
\partial_{t} c^{e f f}+\frac{2}{3} \partial_{x} c^{e f f}+\frac{4}{315} \varepsilon^{2-\alpha} \frac{\partial^{2} c^{e f f}}{\partial x \partial t}=0 \text { for }(x, t) \in \mathbb{R}_{+}^{2} \\
\left.c^{e f f}\right|_{x=0}=c_{f}(t)-\int_{0}^{t} e^{105(z-t)) /\left(2 \varepsilon^{2-\alpha}\right)} \partial_{z} c_{f} d z \\
\partial_{x} c^{e f f} \in L^{2}((0,+\infty) \times(0, T)),\left.\quad c^{e f f}\right|_{t=0}=c_{0}(x) .
\end{array}\right.
$$

\subsubsection{Dispersion in porous media}

In what follows, we review some of the literature contributions aimed at obtaining the macroscopic dispersion tensor starting from the pore scale equations. Dispersion in porous media was studied using the technique of volume averaging in [101, 102, 103], where the upscaled system is obtained by making the ad hoc closure hypothesis that the perturbation of the upscaled concentration is proportional to its gradient. In particular, in [101, 102], Launder's "single point" closure schemes of turbulence modeling is borrowed with the goal of obtaining an effective model for reactive flows in capillary tubes. This approach leads to the correct general form of the effective equations, but it does not produces the effective coefficients. 
The study of the dispersion in porous media using multiple scale expansions was undertaken in a number of contributions. The authors of $[90,104]$ focused at the important case when the Péclet number is of order $O(1 / \varepsilon)$, with $\varepsilon$ the characteristic pore size. In [105], dispersion was studied for various magnitudes of the Péclet number and the reader is also pointed to [106] for a systematic study of the dependency of the dispersion tensor on $\mathrm{Pe}_{T}^{\beta}$.

In [107], various arrangements of periodic arrays of circular cylinders were studied using the two-dimensional flow approximation. It was found that (a) if the cylinders in the array are arranged as the vertices of a square (Cartesian) lattice, then $1.734 \leq \beta \leq 1.761$ and (b) if the cylinders in the array are arranged as the vertices of an hexagonal lattice, then $1.659 \leq \beta \leq 1.82$. For the simpler case of the flow between two parallel corrugated plates, in the same article, the limit Taylor exponent $\beta=2$ was obtained. These results compare also very well with [108]. In the case of random media with non-periodic geometries (e.g., the random Sierpinski carpet) $\beta=1$ and, for reconstructed porous media based on Fontainebleau sandstones, $1.34 \leq \beta \leq 1.56$. For more discussion we refer to [109].

The dispersion effects in porous media could be studied directly by numerical homogenization. We are not aware of the application of the heterogeneous multiscale methods (HMM) to the determination of the dispersion. As already explained, the multiscale finite element method is using the dispersion closure from [60, 61] to calculate the dispersion tensor. Particularly, the molecular diffusion is neglected and the dispersion tensor is set to be a quadratic nonlocal operator in the velocity fluctuation.

For the mathematically rigorous study of the diffusion time regime, the reader can refer to [105, 106], and also [110, 111, 112]. In the latter set of references, two-scale expansions with drift were used to mathematically justify the proposed form of the dispersion tensor, obtained through auxiliary RVE problems. The method of two-scale expansions with drift goes back to [113] and is a well-adapted tool to study not only diffusive transport but also reactive flows at the diffusion time scale through porous media. To fix the ideas, consider the domain $\Omega_{\varepsilon}$, whose unit periodicity cell is depicted in Fig. 1 and a linearization applied to the models proposed in [114], [115], summarized below for the generalized unknowns $u_{\varepsilon}$ and $v_{\varepsilon}$ :

$$
\begin{aligned}
\frac{\partial u_{\varepsilon}}{\partial t}+\operatorname{Pe} \mathbf{v}_{\varepsilon} \cdot \nabla_{x} u_{\varepsilon}=\nabla_{x}^{2} u_{\varepsilon}, & \text { in } \Omega_{\varepsilon} \times(0, T) \\
u_{\varepsilon}(\mathbf{x}, 0)=u^{0}(\mathbf{x}), & \mathbf{x} \in \Omega_{\varepsilon}, \\
-\nabla_{x} u_{\varepsilon} \cdot \mathbf{n}=\frac{T_{A}}{T_{\text {react }}} \frac{\partial v_{\varepsilon}}{\partial t}=\frac{T_{D}}{T_{\text {react }}}\left(u_{\varepsilon}-r_{A D} v_{\varepsilon}\right), & \text { on } \partial \Omega_{\varepsilon} \times(0, T) \\
v_{\varepsilon}(\mathbf{x}, 0)=v^{0}(\mathbf{x}), & \mathbf{x} \in \partial \Omega_{\varepsilon},
\end{aligned}
$$

where Pe is the Péclet number, $T_{D}$ is the characteristic diffusion time, $T_{A}$ is the adsorption time and $T_{\text {react }}$ is the superficial chemical reactions time. The ratio $\mathrm{Da}=T_{A} / T_{\text {react }}$ is Damköhler's number, and we suppose $\mathrm{Da}=O(1 / \varepsilon)$, $\mathrm{Pe}=O(1 / \varepsilon), r_{A D}=O(1)$ and $\frac{T_{A}}{T_{\text {react }}}=O(\varepsilon)$. Furthermore, the transport velocity is defined as

$$
\mathbf{v}_{\varepsilon}(\mathbf{x}, t)=\mathbf{b}\left(\frac{\mathbf{x}}{\varepsilon}\right)
$$

by means of a periodic divergence-free vector field $\mathbf{b}(\mathbf{y})$ satisfying

$$
\max _{\mathbf{y} \in \bar{Y}^{0}}|\mathbf{b}(\mathbf{y})| \leq C, \quad \nabla_{y} \cdot \mathbf{b}(\mathbf{y})=0 \text { in } Y^{0}, \quad \mathbf{b}(\mathbf{y}) \cdot \mathbf{n}(\mathbf{y})=0 \text { on } S^{0} .
$$

Notice that the velocity $\mathbf{v}_{\varepsilon}$ could be associated to any type of incompressible flow. The two-scale expansions with drift consist in expanding the unknowns as

$$
\begin{aligned}
& u_{\varepsilon}(t, \mathbf{x})=\sum_{i=0}^{+\infty} \varepsilon^{i} u_{i}\left(t, \mathbf{x}-\frac{\mathbf{b}^{*}}{\varepsilon} t, \frac{\mathbf{x}}{\varepsilon}\right), \\
& v_{\varepsilon}(t, \mathbf{x})=\sum_{i=0}^{+\infty} \varepsilon^{i} v_{i}\left(t, \mathbf{x}-\frac{\mathbf{b}^{*}}{\varepsilon} t, \frac{\mathbf{x}}{\varepsilon}\right),
\end{aligned}
$$

where the $u_{i}(t, \mathbf{x}, \mathbf{y})$ 's and $v_{i}(t, \mathbf{x}, \mathbf{y})$ 's are functions of the macroscopic variable $\mathbf{x}$ and of the periodic microscopic variable $\mathbf{y} \in Y=(0,1)^{n}$, and the effective drift velocity $\mathbf{b}^{*}$ is an unknown constant vector and its determination is 
part of the problem. The introduction of the drift velocity is motivated by the observation that, for high Péclet and Damkohler numbers, classical two-scale expansions (obtained as the special cases of the two-scale expansions with drift when $\mathbf{b}^{*}=0$ ) lead to a contradiction when solving the equations at the level of the representative elementary volume (REV).

For the mathematical theory of the two-scale expansions with drift we refer to [116] and [111, 112]. Another approach is to use two time scales as in [105]. The drift velocity $\mathbf{b}^{*}$ is calculated by imposing solvability of the REV cell problems through Fredholm's alternative, which yields

$$
\mathbf{b}^{*}=\frac{1}{\phi+\tau / r_{A D}} \int_{Y^{0}} \mathbf{b}(\mathbf{y}) d \mathbf{y},
$$

where $\tau$ is the tortuosity, defined as the total area of the solid interfaces in the REV cell, $\tau=\left|S^{0}\right|$. After mapping back from the moving frame to the fixed coordinate system and setting $\tilde{u}_{\varepsilon}(t, \mathbf{x})=u_{0}\left(t, \mathbf{x}-\left(\mathbf{b}^{*} / \varepsilon\right) t\right)$, we obtain the upscaled problem

$$
\begin{array}{cc}
\left(\phi+\tau / r_{A D}\right)\left(\frac{\partial \tilde{u}_{\varepsilon}}{\partial t}+\frac{\mathbf{b}^{*}}{\varepsilon} \cdot \nabla \tilde{u}_{\varepsilon}\right)-\operatorname{div}\left(A^{*} \nabla \tilde{u}_{\varepsilon}\right)=0 & \text { in } \mathbb{R}^{n} \times(0, T) \\
\tilde{u}_{\varepsilon}(0, x)=\frac{\phi u^{0}(x)+\tau \nu^{0}(x)}{\phi+\frac{\tau}{r_{A D}}} & \text { in } \mathbb{R}^{n}
\end{array}
$$

where $A^{*}$ is the dispersion matrix, derived through the REV cell problems quantities [111, 112]. Fast chemical reactions lead to the quadratic growth of $A_{i j}^{*}$ with respect to the transversal Péclet's number $\mathrm{Pe}_{T}=\varepsilon \mathrm{Pe}$. In the absence of chemical reactions, $A_{i j}^{*}=O\left(\mathrm{Pe}_{T}^{1.7}\right)$, in agreement with [107].

Based on the analysis presented in [110, 111, 112], it is also possible to draw the conclusion that the dispersion tensor proposed by Peaceman [2], namely [5], can be safely used in applications. Moreover, in practical applications, it is important to derive the dispersion tensor from Darcy's flow equation in a heterogeneous porous medium, a problem that was addressed with mathematical rigor in [117, 118]. For a practical discussion, see also [119, 120, 121, 122, 19].

We conclude this subsection with a comment: The validity of the methods presented here for computing the dispersion tensor depend strongly on the Péclet number. If it is large, but not too large, we are in the Taylor dispersion regime and the tools of homogenization allow to incorporate dispersion effects. If the Péclet number is too large, we enter the turbulent dispersion regime and homogenization is not appropriate any more.

\subsection{Gravity-driven (or density-driven) flows}

In fluid mechanics, density-driven flows are typically analyzed through the study of Rayleigh-Taylor instabilities in stratified incompressible inviscid fluids. The literature is rich on this subject and we refer to [62, 63] and references therein. Instabilities of Rayleigh-Taylor type in viscous porous media flows are referred to as Saffman-Taylor instabilities, after the names of the authors of [20]. An analogy between the incompressible Euler equation and Darcy's law is made, in order to study a system consisting of a lower fluid of density $\rho_{1}$ and viscosity $\mu_{1}$ and an upper fluid of density $\rho_{2}$ and viscosity $\mu_{2}$. For the sake of simplicity, the permeability tensors are often assumed isotropic, and the scalar permeabilities values are denoted $\mathrm{K}_{1}$ and $\mathrm{K}_{2}$, respectively. If the base velocity of the fluid is vertical, that is $\mathbf{w}=w \mathbf{e}_{3}$ with $w=|\mathbf{w}|$, then the flow is stable if

$$
\left(\frac{\mu_{1}}{k_{1}}-\frac{\mu_{2}}{k_{2}}\right) w+g\left(\rho_{1}-\rho_{2}\right) \geq 0 .
$$

For more details see [123, 124, 125, 126, 11].

Natural free convection has now been studied for over a century and most works rely on an analogy between density-driven flows through porous media and thermal flows (see [127, 32] for a more detailed discussion on physical mechanisms, and [128, 129, 130, 131] for a mathematical introduction). Natural free convection represents an effective mechanism for enhancing heat and solute transport by increasing the size of characteristic spatial scales and shortening the characteristic time scales with respect to simple diffusion. Recent industrial applications have led to an increased interest in density driven flow and free convection processes in groundwater systems and other natural porous media. 
In classical references (see, e.g., [128]), natural convection is described by the dimensionless system

$$
\begin{aligned}
\mathbf{u}+\nabla p & =\operatorname{Ra} T \mathbf{e}_{3}, \\
\nabla \cdot \mathbf{u} & =0, \\
\frac{\partial T}{\partial t}+\mathbf{u} \cdot \nabla T & =\Delta T,
\end{aligned}
$$

where we have introduced the Rayleigh number

$$
\mathrm{Ra}=\frac{\mathrm{K} \rho_{f} g L \alpha\left(T_{1}-T_{2}\right)}{\chi \mu} .
$$

Here $\mathrm{K}$ is the permeability of the porous medium, $T_{1}>T_{2}$ are two temperatures, $\alpha$ is the thermal expansion coefficient, $\chi$ is the thermal conductivity of the porous medium, $\mu$ is the viscosity of the fluid, $L$ is a characteristic length of the domain and $\rho=\rho_{f}\left(1-\alpha\left(T-T_{0}\right)\right)$ is the fluid density. We observe that system 67a)-67d corresponds to system (42b)-42c) with constant viscosity $\mu$.

In [128], it was established that, for small Rayleigh numbers, a unique, regular and analytic solution exists for the system 67a)-67d. It was also shown that this unique solution is stationary for small Rayleigh numbers, and that instabilities occur for large Rayleigh numbers.

The theory of unstable convective motion in porous media can be considered as well understood, for the many references that have been contributed on the subject. Large contrasts in fluid density, due to differences in solute concentrations and temperatures, can cause fingering instabilities (unstable free convection). Following [34], when the critical condition for the onset of free convection is exceeded, nearly square circulations will develop with a steady state fingering wavelength equal to twice the thickness of the layer in which convection occurs.

We focus our interest on the case of large contrasts in fluid density due to differences in solute concentrations and compressibility effects. Following [132, 35, 36], the model is given by system (10, , 35a and 35b):

$$
\begin{aligned}
& \rho\left(\partial_{t}\left(\phi c_{i}\right)+\mathbf{u} \cdot \nabla c_{i}\right)=\nabla \cdot\left(\phi \rho \mathbf{D} \nabla c_{i}\right)+q_{i}-c_{i} q, \quad i=1, \ldots, N_{c}-1, \\
& D_{\phi} \rho=\partial_{t}(\phi \rho)+\mathbf{u} \cdot \nabla \rho=q-\rho \nabla \cdot \mathbf{u}, \\
& \mathbf{u}=-\frac{\mathbf{K}}{\mu}(\nabla p-\rho \mathbf{g}) .
\end{aligned}
$$

Let $\left\{p_{0}, \mathbf{u}_{0}, c_{0}\right\}$ be a smooth stationary solution for system 67a-67d). The linearized problem around $\left\{p_{0}, \mathbf{u}_{0}, c_{0}\right\}$ reads

$$
\begin{aligned}
& \delta \mathbf{u}=-\frac{\mathbf{K}}{\mu_{0}}\left(\nabla(\delta p)-\left(\frac{\partial \rho_{0}}{\partial p} \delta p+\sum_{j=1}^{N_{c}-1} \frac{\partial \rho_{0}}{\partial c_{j}} \delta c_{j}\right) \mathbf{g}\right)+\frac{\mathbf{K}}{\mu_{0}^{2}}\left(\sum_{j=1}^{N_{c}-1} \frac{\partial \mu_{0}}{\partial c_{j}} \delta c_{j}\right)\left(\nabla p_{0}-\rho_{0} \mathbf{g}\right), \\
& \rho_{0}\left(\partial_{t}\left(\phi \delta c_{i}\right)+\mathbf{u}_{0} \cdot \nabla \delta c_{i}+\mathbf{v} \cdot \nabla c_{i}^{0}\right)+\left(\frac{\partial \rho_{0}}{\partial p} \delta p+\sum_{j=1}^{N_{c}-1} \frac{\partial \rho_{0}}{\partial c_{j}} \delta c_{j}\right) \\
& =\nabla \cdot\left(\phi \rho_{0} \mathbf{D}\left(\mathbf{u}_{0}\right) \nabla \delta c_{i}+\phi\left(\frac{\partial \rho_{0}}{\partial p} \delta p+\sum_{j=1}^{N_{c}-1} \frac{\partial \rho_{0}}{\partial c_{j}} \delta c_{j}\right) \mathbf{u}_{0} \nabla\left(c_{0}\right)_{i}+\phi \rho_{0}\left(\mathbf{v} \cdot \mathcal{D}\left(\mathbf{u}_{0}\right)\right) \nabla\left(c_{0}\right)_{i}\right)-\delta c_{i} q, \quad i=1, \ldots, N_{c}-1,
\end{aligned}
$$

$$
\begin{gathered}
\partial_{t}\left(\phi\left(\frac{\partial \rho_{0}}{\partial p} \delta p+\sum_{j=1}^{N_{c}-1} \frac{\partial \rho_{0}}{\partial c_{j}} \delta c_{j}\right)\right)+\mathbf{u}_{0} \cdot\left(\frac{\partial \rho_{0}}{\partial p} \delta p+\sum_{j=1}^{N_{c}-1} \frac{\partial \rho_{0}}{\partial c_{j}} \delta c_{j}\right)+\delta \mathbf{u} \cdot \nabla \rho_{0} \\
=q-\rho_{0} \nabla \cdot \delta \mathbf{u}-\left(\frac{\partial \rho_{0}}{\partial p} \delta p+\sum_{j=1}^{N_{c}-1} \frac{\partial \rho_{0}}{\partial c_{j}} \delta c_{j}\right) \nabla \cdot \mathbf{u}_{0}
\end{gathered}
$$


where $\{\delta p, \delta \mathbf{u}, \delta c\}$ is the perturbation, $\rho_{0}=\rho\left(p_{0}, \mathbf{c}_{0}\right), \mu_{0}=\mu\left(\mathbf{c}_{0}\right), \frac{\partial \rho_{0}}{\partial p}=\frac{\partial \rho}{\partial p}\left(p_{0}, \mathbf{c}_{0}\right), \frac{\partial \rho_{0}}{\partial c_{j}}=\frac{\partial \rho}{\partial c_{j}}\left(p_{0}, \mathbf{c}_{\mathbf{0}}\right), \frac{\partial \mu_{0}}{\partial c_{j}}=\frac{\partial \mu}{\partial c_{j}}\left(\mathbf{c}_{0}\right)$ and $\mathcal{D}_{i j k}=\partial_{u_{i}} D_{j k}\left(\mathbf{u}_{0}\right)$. We are not aware of any rigorous mathematical study of 69a)-69c) and/or the corresponding spectral problem. We note that adverse viscosity and density contrasts could create rather complex scenarios. Using the analogy with thermal flow systems, the analysis from [128] would apply in principle to the Boussinesq approximation of gravitational instabilities. Even in this case though, the rigorous analysis is available only for small Rayleigh numbers. With regard to dissolution-diffusion-convection processes linked to carbon dioxide sequestration and the linear stability analysis under incompressibility and Boussinesq approximations, we refer to [133, 134, 135, 136, 11, 137, 138, 139, 140, 141, 142], where analytical calculations are often complemented with numerical simulations.

\subsection{Estimates for the mixing zone and tip-velocity of leading fingers}

Of particular interest is the prediction of the spreading and growth of the mixing zone. Several empirical methods were proposed and models of widespread use are due to [143, 144, 132]. In particular, the correlated upscaling technique proposed by Todd et al. [144] has been widely used in commercial reservoir simulators to mimic the effect of small-scale fingering behavior on coarse grids, and to account for how a large mobility ratio $M$ would enhance viscous and gravitational instabilities.

The mixing zone is a region where complex patterns form as a result of the mixing of fluids of largely different viscosities and/or densities. As already mentioned, finger-like structures are produced when, for example, the viscosity in a displacing component of a fluid is much lower than in the displaced component. Then the injected (displacing) component has the tendency to go around and overtake the displaced component. The same can be argued when it is density rather than viscosity contrast to drive the formation of fingers. The dynamics of viscous fingers can be rather complex, and typically, in experiments, shielding, spreading and splitting of the fingers is observed. The experimental results from the seminal work of Saffman and Taylor [20] showed that, after the onset of instability, a single finger appeared to dominate the flow. It is the shielding effect: a finger moves slightly ahead of its neighbors and shields them from further growth. For miscible flows, the transverse dispersion can disturb shielding. Experiments indicate that the dominant finger is a stable flow pattern, until a tip-splitting instability (bifurcation of the finger into two or more fingers) occurs. The dynamics of tip-splitting is influenced by the (high) Péclet number in miscible flows.

In [145], Wooding presented the modeling and experimental study of the Rayleigh-Taylor fingering instability for an incompressible two-component mixture. While the early stages of the flow evolution are governed by the equations of linear stability theory, a much more challenging question is the study of the dynamics of the coherent fingers in subsequent stages. A detailed qualitative study was undertaken by Menon and Otto in [146]. The dimensionless form of the problem analyzed by Wooding [145] in a Hele-Shaw cell is

$$
\begin{array}{rll}
\partial_{t} s+\mathbf{u} \cdot \nabla s-\Delta s=0 & \text { in } & (0, L)^{n_{d}-1} \times \mathbb{R}, \\
\nabla \cdot \mathbf{u}=0 & \text { in } \quad(0, L)^{n_{d}-1} \times \mathbb{R}, \\
\mathbf{u}+\nabla p+s \mathbf{e}_{3}=0 & \text { in } \quad(0, L)^{n_{d}-1} \times \mathbb{R} .
\end{array}
$$

In passing to the dimensionless form of the equations, one finds that $L$ is proportional to the Péclet number and we write for simplicity, and without loss of generality, $L=$ Pe. System (70a)-(70c) corresponds to (42a)- 42c). The following observations were made regarding the mixing zone:

(i) The length $a(t)$ of the mixing zone does not depend on the Péclet (Pe) number Pe and scales typically with $t$.

(ii) The finger zone width $b(t)$ broadens as $O(\sqrt{t})$.

In [146], the (negative gravitational) energy $E(t)$ and the mean perimeter $P(t)$ were introduced:

$$
\begin{aligned}
& E(t)=\int_{\mathbb{R}}\left(s_{0}(z)-\bar{s}(t, z)\right) d z, \\
& P(t)=L^{-n_{d}+1} \int_{\mathbb{R}} \int_{(0, L)^{n_{d}-1}}|\nabla s| d x=\int_{0}^{1} \mathcal{H}^{n_{d}-1}\left(s^{-1}(c)\right) d c,
\end{aligned}
$$


where

$$
\bar{s}(t, z)=L^{-n_{d}+1} \int_{(0, L)^{n_{d}-1}} s d x,
$$

and it was proved that

$$
\limsup _{t \rightarrow \infty} \frac{E(t)}{t^{2}} \leq \frac{1}{6} \quad \text { and } \quad \limsup _{t \rightarrow \infty} \frac{1}{t^{2}} \int_{0}^{t} P^{2}(\tau) d \tau \leq \frac{\pi}{9} .
$$

Here $s(t, x)$ is compared to the rarefaction wave (entropy solution) associated with the Riemann problem

$$
\begin{aligned}
\partial s_{1}+\partial_{x}\left(s_{1}\left(1-s_{1}\right)\right) & =0, \\
s_{1}(0, z) & =s_{0}(z),
\end{aligned}
$$

given by

$$
s_{1}(t, z)= \begin{cases}0, & \text { for } \quad z<-t, \\ (z+t) /(2 t), & \text { for } \quad-t \leq z \leq t, \\ 1, & \text { for } z>t .\end{cases}
$$

For $s_{1}$, we have $a(t)=2 t$ and $E(t)=t^{2} / 6$. The estimate for $P(t)$ in 72 is the integrated version of

$$
P(t) \leq \frac{\sqrt{2 \pi}}{3} \sqrt{t},
$$

which indicates that $b(t) \leq C \sqrt{t}$. Nevertheless the estimate $a(t) \leq 2 t$ is twice the experimental result $a(t) \leq t$ and the authors of [146] studied the diffusive slowdown responsible for a reduction of the length of the mixing zone by a factor 2. With a similar goal, in [145], a version of system (70a)-70c] based on lubrication theory was also introduced, namely

$$
\begin{aligned}
& \partial_{t} s+\mathbf{u} \cdot \nabla s=\Delta s \quad \text { in } \quad(0, L)^{n_{d}-1} \times \mathbb{R}, \\
& \nabla \cdot \mathbf{u}=0 \quad \text { in } \quad(0, L)^{n_{d}-1} \times \mathbb{R}, \\
& \mathbf{u}=(v, w) \quad \text { in } \quad(0, L)^{n_{d}-1} \times \mathbb{R}, \\
& w=\bar{s}-s \quad \text { in } \quad(0, L)^{n_{d}-1} \times \mathbb{R} \text {. }
\end{aligned}
$$

In [146] it was proved that if $s(t, x)$ is a classical solution to 75a-(75d) with continuous initial data $s(0, x), 0 \leq s \leq 1$, and such that

$$
\lim _{z \rightarrow-\infty} \max _{y} s(0, y, z)=0 \quad \text { and } \quad \lim _{z \rightarrow \infty} \min _{y} s(0, y, z)=1,
$$

then for any $c>1 / 2$

$$
\lim _{t \rightarrow \infty} \max _{y} s(t, y,-c t)=0 \quad \text { and } \quad \lim _{t \rightarrow \infty} \min _{y} s(t, y, c t)=1 .
$$

The pointwise estimates (77) show that the mixing zone does not spread faster than $a(t)=t$, confirming experimental observations from [145] and the numerical experiments from [147], in which $a(t)=0.892 t$. This result also shows that the bounds are achieved by a solution that has sharp gradients at the tip of the finger and does not satisfy the Lax entropy condition. For different notions of entropy solution applying to the conservation laws of porous media flows we refer to [148, 149].

In [150], Menon and Otto addressed the question of the diffusive slowdown for a general miscible flow model, represented by the lubricated Boussinesq approximation of the miscible flows considered in [145]

$$
\begin{aligned}
& \partial_{t} s+\mathbf{u} \cdot \nabla s=\Delta s \quad \text { in } \quad(0, L)^{n_{d}-1} \times \mathbb{R}, \\
& \nabla \cdot \mathbf{u}=0 \quad \text { in } \quad(0, L)^{n_{d}-1} \times \mathbb{R}, \\
& \mathbf{u}=(v, w) \quad \text { in }(0, L)^{n_{d}-1} \times \mathbb{R}, \\
& w=\alpha(\bar{s})(\bar{s}-s) \quad \text { in } \quad(0, L)^{n_{d}-1} \times \mathbb{R} .
\end{aligned}
$$


where $\alpha(\bar{s})=m_{h} m_{l} /\left(m_{h} \bar{s}+m_{l}(1-\bar{s})\right), m_{l}$ and $m_{h}$ are positive constants. Generalizing results from [146] to the case of a particular concentration-dependent viscosity $\alpha$, Menon and Otto [150] proved that, if $s(t, x)$ is a classical solution of 75a-75d with continuous initial data $s(0, x), 0 \leq s \leq 1$, and such that

$$
\lim _{z \rightarrow-\infty} \max _{y} s(0, y, z)=0 \text { and } \lim _{z \rightarrow \infty} \min _{y} s(0, y, z)=1
$$

then, for any $c_{h}>m_{h} / 2$ and $c_{l}>m_{l} / 2$,

$$
\lim _{t \rightarrow \infty} \max _{y} s\left(t, y,-c_{h} t\right)=0 \quad \text { and } \quad \lim _{t \rightarrow \infty} \min _{y} s\left(t, y, c_{l} t\right)=1 .
$$

In the limit $\mathrm{Pe} \rightarrow \infty$, the solution to the hyperbolic problem is the shock wave solution

$$
s^{*}(t, z)= \begin{cases}0, & \text { for } \quad 2 z<-m_{h} t, \\ m_{l} /\left(m_{l}+m_{h}\right), & \text { for } \quad-m_{h} t \leq 2 z \leq m_{l} t, \\ 1, & \text { for } 2 z>m_{l} t\end{cases}
$$

Also in this case, and in analogy to the work of Menon and Otto [146] on density-driven instabilities, [81] is a nonentropic solution (with respect to the canonical Lax entropy).

Yortsos and Salin [151] considered a miscible displacement in the absence of gravity in a two-dimensional porous medium of rectilinear geometry. A fluid, initially occupying the porous medium, is displaced at constant injection rate by another fluid of lower viscosity $\left(\mu_{1}>\mu_{2}\right)$. After instabilities develop, the resulting displacement reaches an asymptotic regime, which in [152] is termed the state of transverse flow equilibrium (TFE). The corresponding model is described by the TFE equations

$$
\begin{aligned}
\frac{\partial c}{\partial t}+\frac{\lambda(c)}{\int_{0}^{1} \lambda(c) d y} \frac{\partial c}{\partial x}+w \frac{\partial c}{\partial y} & =\frac{1}{\mathrm{Pe}} \Delta c, \\
\frac{\partial u}{\partial x}+\frac{\partial w}{\partial y} & =0,
\end{aligned}
$$

where

$$
\begin{aligned}
& u=\frac{k \lambda}{\int_{0}^{1} k \lambda d y}, \\
& \lambda=\mu_{1} / \mu(c)=\exp (\ln M c) .
\end{aligned}
$$

Averaging $82 \mathrm{a}-182 \mathrm{~d}$ ) with respect to $y$ and applying a closure yields an equation for $y$-averaged concentration $\bar{c}$ :

$$
\frac{\partial \bar{c}}{\partial t}+\frac{\partial}{\partial x} \frac{M \bar{c}}{M \bar{c}+1-\bar{c}}=\frac{1}{\mathrm{Pe}} \Delta \bar{c}
$$

The entropy solution in the high Péclet limit of problem $\sqrt{83}$ is a rarefaction wave with a leading velocity $M$ of the mixing zone and a trailing velocity $1 / M$. However, experiments and numerical simulations of the full problem, have conclusively shown that the true leading velocity is smaller than $M$ and that the trailing-edge velocity is larger than $1 / M$. In [151], the approach from [146] was applied and the "lubricated" problem was considered. Namely,

$$
\frac{\partial \bar{c}}{\partial t}+\frac{\partial}{\partial x}\left(\bar{c}^{2}-\bar{c}\right)=\frac{1}{\mathrm{Pe}} \Delta \bar{c} .
$$

Using the approach from [146], it was found that the leading edge velocity cannot exceed $(M-1)^{2} /(M \ln M)$. Again, it corresponds to a non-entropic solution in the limit $\mathrm{Pe} \rightarrow \infty$ and, again, we observe the failure of the entropy solution approach in describing the actual evolution of the transversely averaged profile. We also note that non-classical shocks of the type introduced in [149] could potentially reconcile this problem. 


\section{Overview of computational aspects and challenges}

\subsection{Time integration and equation coupling strategies}

Many time integrators have been proposed to advance in time the governing equations of transport in porous media flows: Backward Differentiation Formulas (BDF) of first (BDF1, i.e., Backward Euler), second (BDF2) and higher order, Runge-Kutta methods (either implicit or explicit), and the midpoint method, are among the most commonly used. One aspect of importance is that, in unstable flow computations, the accuracy of the methods in space and time have to be matched, since they both contribute to the overall fidelity of the simulation. This goal can be achieved with different strategies: one option is to match the order of accuracy of the space and time discretizations, while another is to use lower-order time stepping strategies with respect to the spatial discretization and reduce the size of time steps. While the second approach may be in principle regarded as less efficient, in practice the strong nonlinearities that need to be resolved in viscous fingering problems may at times pose severe restrictions on the time steps, and the full benefit of matching the orders of accuracy in space and time may not be achieved.

Before delving into the discussion of various options available for spatial discretization, it is also important to discuss strategies for coupling the flow (mass conservation) equation and the (species) transport equations. The most frequently encountered approaches are IMPES coupling, fully implicit coupling, and sequential coupling. These three classes are standard in the case of miscible displacement computations, and are also commonly used in multiphase flows. A general overview with an analysis can be found in [153, 154]. We introduce now the notation that will be used throughout this section. Let $N \in \mathbb{N}$ be the number of time steps, and $\delta t^{n+1}=t^{n+1}-t^{n}$ the time step. Furthermore, for a time dependent function, we denote $\phi^{n}=\phi\left(t^{n}\right)$ and $\phi^{\delta t}=\left\{\phi^{n}\right\}_{n=0}^{N}$. In what follows, for the sake of simplicity and without loss of generality, we describe the coupling strategies in semi-discretized form and in the context of first-order time integrators. High-order time integration can also be combined with each specific approach to equation coupling, with implementations that, although more complex, follow very closely the procedures relative to lower-order time integrators.

\subsubsection{IMPES coupling}

The IMPES scheme stands for implicit pressure and explicit saturation (or, in our case, concentration). As the name suggests, the IMPES scheme involves solving (implicitly) first an equation for the pressure at $p^{n+1}$ in the timestep sequence, by lagging all concentration-dependent variables to the previous time level $t^{n}$. If the pressure system is compressible, the time derivative of pressure is approximated by the backward difference quotient $\left(p^{n+1}-p^{n}\right) / \delta t$. Then one solves explicitly for $c^{n+1}$, using the field $p^{n+1}$ just updated. Namely, we have

$$
\begin{array}{r}
\frac{\tilde{\phi}^{n+1}-\tilde{\phi}^{n}}{\delta t}+\nabla \cdot \tilde{\mathbf{u}}^{n+1}=q^{n+1}, \\
\tilde{\mathbf{u}}^{n+1}=-\tilde{\boldsymbol{\kappa}}^{n} \nabla p^{n+1}, \\
\frac{\left(\tilde{\phi} c_{i}\right)^{n+1}-\left(\tilde{\phi} c_{i}\right)^{n}}{\delta t}=\nabla \cdot\left(\tilde{\phi}{ }^{n} \mathbf{D}\left(\mathbf{u}^{n+1}\right) \nabla c_{i}^{n}-\tilde{\mathbf{u}}^{n+1} \nabla c_{i}^{n}\right)+q_{i}^{n+1} .
\end{array}
$$

The key to an IMPES scheme is the absence of concentration time derivatives in the pressure equation. IMPES is efficient since only the pressure is solved for implicitly. However, the concentration transport system is subject to a CFL (Courant-Friedrichs-Lewy) stability condition, restricting the time step size relative to the magnitude of the velocity and the spatial mesh size. In realistic reservoir engineering applications, steep solution fronts can form in proximity of wells, and finely resolved grids may be required, with more severe limitations on the time step size. For this reason, IMPES coupling is not widely used in industrial problems.

\subsubsection{Fully implicit coupling}

In the fully implicit procedure, a nonlinear system for $p$ and $c$ is solved simultaneously by Newton's method:

$$
\begin{aligned}
\frac{\tilde{\phi}^{n+1}-\tilde{\phi}^{n}}{\delta t}+\nabla \cdot \tilde{\mathbf{u}}^{n+1} & =q^{n+1}, \\
\frac{\left(\tilde{\phi} c_{i}\right)^{n+1}-\left(\tilde{\phi} c_{i}\right)^{n}}{\delta t}-\nabla \cdot\left(\tilde{\phi}^{n+1} \mathbf{D}\left(\mathbf{u}^{n+1}\right) \nabla c_{i}^{n+1}-\tilde{\mathbf{u}}^{n+1} \nabla c_{i}^{n+1}\right) & =q_{i}^{n+1} .
\end{aligned}
$$


In the case of linear systems of equations, fully implicit time stepping eliminates the stability constraints (i.e., the CFL condition that affects IMPES methods). In the case of general nonlinear systems it is not always possible to prove that implicit time stepping completely eliminates a CFL condition. For example, the Newton's method is not guaranteed in general to converge unless the initial estimate, the solution at the previous time step, is sufficiently close to the solution at the next time instant. However, fully coupled implicit methods, in practice, allow for much larger time steps with respect to IMPES methods, and the overall numerical solution procedure enjoys much improved reliability and robustness, especially when considering complex systems. In the case of miscible displacement, a fully implicit scheme was analyzed in [155]. At the same time, the cost of solving the fully coupled system may be high in certain applications, as is the case, for example, when solving multiphase problems with nonlinear relative permeability coefficients in the convection terms, with saturation-dependent capillary pressures, or with variable density and porosity in the accumulation terms.

\subsubsection{Sequential coupling}

A sequential method solves both pressure $p$ and transport $c$ implicitly, but in a decoupled scheme. Thus, either $c$ in flow system or $\mathbf{u}$ in the transport system must be time lagged or extrapolated in time to decouple the equations. Usually, $\mathbf{u}$ is extrapolated in the transport system since $\mathbf{u}$ is expected to be smoother in time than $c$. Thus, first the system of equations for the $c_{i}$ 's is solved, followed by the equation for $p$ :

$$
\begin{aligned}
\frac{\left(\tilde{\phi} c_{i}\right)^{n+1}-\left(\tilde{\phi} c_{i}\right)^{n}}{\delta t}-\nabla \cdot\left(E \tilde{\phi}^{n+1} \mathbf{D}\left(E \mathbf{u}^{n+1}\right) \nabla c_{i}^{n+1}-E \tilde{\mathbf{u}}^{n+1} \nabla c_{i}^{n+1}\right) & =q_{i}^{n+1}, \\
\frac{\tilde{\phi}^{n+1}-\tilde{\phi}^{n}}{\delta t}+\nabla \cdot \tilde{\mathbf{u}}^{n+1} & =q^{n+1},
\end{aligned}
$$

where $E \mathbf{u}^{n+1}$ and $E \tilde{\phi}^{n+1}$ are linear extrapolations for $\mathbf{u}^{n+1}$ and $\tilde{\phi}^{n+1}$, respectively, from previous values. The stability and convergence rate estimates established for sequential procedures also apply to the fully implicit method, provided the Newton iteration converges to a unique solution, as discussed in detail in [153].

\subsection{Grid dependence and grid orientation effects in numerical computations}

In general, grid dependence of the result is an often overlooked aspect of numerical simulations. In the specific case of unstable flows in porous media, grid dependence of the results can be particularly exacerbated through nonlinear couplings and transport mechanisms, as will be shown in Section 5.2.3 This is because, as opposed to the case of a stable physical system, in an unstable flow there is a natural tendency for slight differences and discrepancies between solutions to grow uncontrollably over time. Grid orientation effects can become more severe in multi-dimensional problems with large mobility ratios and heterogeneous coefficients, as discussed in [156, 157, 158, 159, 160, 144].

In what follows, we will devote some of the discussion to evaluating how various algorithms perform when changes in grid orientation are applied to computations, as shown, for example in Figure 2 for the canonical quarter five-spot problem.

In general, however, grid orientation problems are tightly connected with the invariance under rotation of the discrete equations and the quality of the numerical discretization in representing gradients. For example, a two-point finite volume discretization of the gradients is particularly prone to mesh orientation effects, while multi-point finite volume and higher-order finite element discretizations (i.e., second and higher) are particularly effective in minimizing grid orientation and dependency effects. Similarly, particular care needs to be placed in assuring that the discretization of the velocity-dependent dispersion tensor is rotationally invariant, or at a minimum, nearly invariant (i.e., with a high-order invariance error).

In this perspective, we would like to offer some comments on the many efforts have been made to reduce grid orientation and dependency effects. Grid refinement is usually not effective to reduce grid orientation effects, since, typically, it does not modify the alignment of elements, nor the quality of discretized gradients. Improved solutions have been obtained applying multi-point (e.g., nine-point) formulations in finite volume methods, as well as two-point upstream weighting. The use of curvilinear coordinates has resulted in mixed results, somewhat problem specific, in the sense that through mappings it is possible to improve the accuracy of gradients, but not their invariance properties. The use of fully unstructured triangulations has resulted in somewhat improved solutions, although some of the outcomes depend still on the quality of gradient discretizations. Improving integration strategies for the full dispersion tensor in finite element methods has had a positive impact on the quality of simulations. 
Production well

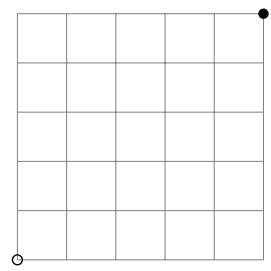

Injection well

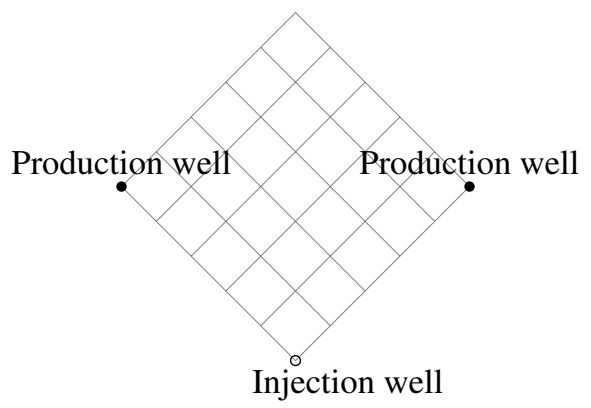

(b) Parallel Mesh

(a) Diagonal Mesh

Figure 2: An example of two grid configurations in a quarter five-spot problem. Grid orientation effects may lead to vastly different computational results of flow instabilities.

\subsection{Discrete local and global conservation}

In porous media flow simulation, numerical local and global conservation take a particularly important role, especially when considering heterogeneities in the medium (faults, layering of the subsurface, etc.) A conservation statement takes the general form of a budget, in which the rate of change of a certain quantity, for example the mass, equals the sum of the inflow, outflow and sources/sinks of that quantity over a certain numerical control volume, namely:

$$
\text { Rate of change }=\text { Inflow }- \text { Outflow }+ \text { Sources } .
$$

Depending on the size of the control volume considered, statements can be made about global conservation (if the control volume corresponds to the entire computational domain), or local conservation (if the control volume corresponds to a small portion of the computational domain, for example a computational cell/element or a patch of cells/elements).

Because of the specific nature and behavior of porous media flows, numerical issues related to conservation can be quite subtle. For example, let us consider the case of how a certain type of local conservation statement can impact numerical results. Conservative finite difference methods, cell-centered finite volume methods, mixed finite element methods, interior penalty and discontinuous Galerkin methods satisfy local conservation statements in which the control volume is the element or cell in the discretization. These methods tend to be very effective in porous media flow computations, since typically computational grids/meshes are generated so that edges/faces are aligned with interior interfaces and medium discontinuities. Since this is the typical setup, although locally conservative, continuous Galerkin methods and, to a certain extent, node-centered finite volumes do not provide conservation statements as effective and useful as element-centered discretizations. In the past, there has been some discussion about whether or not continuous finite elements are locally conservative. A positive answer was given in [161], connecting the conservation statement in fluid problems to the force balance statement in mechanics problems (continuous finite elements are well-known to discretely balance nodal forces). However, as also discussed in [162], this is not a sufficient condition for high-quality solution and avoidance of spurious oscillations. In fact, the statement of conservation of typical continuous Galerkin methods involves the patch of elements surrounding a node, and this patch can span across material interfaces. Hence, oscillations may still appear in continuous Galerkin methods as the result of rough changes in coefficients.

On a related note, continuous finite element methods do not allow for tangential discontinuities in the mass fraction component of the solution. As a consequence, when discontinuities in the subsurface permeabilities, conductivities, or mobilities are present, spurious Gibbs oscillations are produced in the continuous approximate solutions. Although this issues is particularly problematic in the immiscible displacement case, also in the miscible case oscillations can be produced every time a rapid variation of equation coefficients occurs (e.g., in the case of strong nonlinearities). We refer to Sections 4.1 and 4.2 for more details, but to a certain degree, we can summarize the situation with the catchphrase that in porous media flow simulations, local/global conservation is paramount, but not all discrete conservation statements are equally effective. 


\subsection{Discrete sum compatibility principle between the flow and transport equations}

Recently, Scovazzi et al. [158] introduced the discrete sum compatibility principle between the equations of transport for the species and the flow equation for the pressure. The key point here is the realization that, in the continuum, the sum of the transport equations for the component mass fractions $c_{i}$, namely (18), is given by the flow equation [19). This is a consequence of the fact that the mass fractions $c_{i}$ add up to unity. In [158], it was suggested that also the numerical discretization of equations (18) and (19) satisfy the same property, that is, the sum of the discretized approximations to (18) add up to the discretized form of (19): we use this statement as the definition of the discrete sum compatibility (DSC) principle.

The simplest possible strategy to satisfy the DSC principle, is to use the exact same discretization strategy for both [18) and [19], as suggested in [157, 158], and demonstrated in the case of an upwind-biased discontinuous Galerkin method. In that case, it was found that it becomes essential, in order to preserve the DSC principle, to use upwind bias in the discrete Laplace-like operators in the flow equation, since they are obtained as the sum of convective operators in the transport equations. More details about this specific algorithm will be given in Section 5.2.3. where the variational equations are specifically discussed, for an upwind-biased symmetric interior penalty formulation.

We stress however, that the DSC principle is not tied to a specific finite element method or a class of methods, but can be applied in very general terms, as long as the discretized flow equation is (discretely) equivalent to the sum of the discretized transport equations. Under this premise, the DSC principle is not limited in applicability to just finite elements, but can also be applied to finite difference and finite volume discretizations. At the same time, it can be verified that the DSC principle is satisfied by numerical formulations already presented in the literature. For example, the incomplete interior penalty Galerkin formulations in [162, 163] satisfy the DSC principle.

We would also like to mention the principle of compatibility between the flow and transport discretizations introduced in [162], according to which a numerical method for the flow and another for the transport are compatible if a variational extension of the statement of local mass conservation is satisfied. This compatibility statement is very valuable in understanding which pairs of discretizations can be safely coupled without producing spurious sources/sinks of mass in the numerical equations.

\subsection{Algebraic solvers}

The solution of the algebraic systems arising in coupled flow and transport in porous media is an area of very active research. Typical computational scenarios in the case of miscible displacement may involve nonlinear systems of partial differential equations with rough coefficients produced by heterogeneous permeabilities, velocity dependent dispersion, high mobility ratios, and concentrated nonlinear point sources/sinks. It would be possible to write an entire monograph only on this subject, especially on how the presence of rough coefficients has led to many different decoupling strategies for the preconditioning of the final algebraic system. Specific approaches to the solution of the flow equations have included preconditioned conjugate gradient methods, domain decomposition methods (additive Schwartz, load balancing), multiscale methods[164, 56] and algebraic/geometric multigrid methods. We refer the reader to [165, 166, 167, 168, 169, 170] for recent contributions and classical references therein.

\subsection{Mesh adaptivity}

The inherent nature of unstable miscible displacement flow tend to produce complex flow patterns and very large gradients in the solution (especially for the species concentrations/mass fractions). Other situations in which mesh refinement is important is in proximity of wells, which negatively affect the regularity of solutions. Mesh adaptation seems then a natural option in the development of modern algorithms: by automatically coarsening and refining the computational grid, the overall number of degrees of freedom can be kept under control without precluding the overall accuracy of the method. Automatic mesh adaptation is driven by error indicators or estimators, and we refer to the work in [171, 172, 173] for additional details about the ongoing work in the realm of porous media flow simulations. Mesh adaptation is performed through appropriate mesh modification techniques, which vary in nature depending on the topology of the elements. For example, edge swapping or flipping are common on unstructured grids of tetrahedral cells, while strategies relying on hanging nodes are more common in hexahedral and logically structured grids. When mesh adaptation is combined with domain decomposition, nonmatching grids are also very frequently encountered. In general, mesh adaptivity for continuous and discontinuous Galerkin methods tend to be more flexible and easier to implement than for mixed finite element methods. Particularly, discontinuous Galerkin methods have 
some important advantages over continuous methods when hanging nodes are present in the adapted grid. For a more recent contribution on these topics, including an overview of the current state of the art in mesh adaptation, we refer the reader to the recent adaptive simulations on miscible displacement flow in [174].

\section{Numerical modeling}

Miscible displacement flow has been the object of intense study through computational simulation. In this section, we provide an overview on the numerical methods that have been successfully applied to enhanced oil recovery and viscous fingering phenomena. In the discussion to follow, we have separated the presentation of algorithms for flow and transport, since often times these have been interchangeably combined in the literature to date. Emphasis in the discussion will be given to variational methods, which are the key focus of the present article.

First, we introduce our general notations. Let $\mathcal{T}_{h}$ be a shape-regular triangulation (in the sense of Ciarlet) constituted by a family of non-overlapping $n_{d}$-partitions/elements $\Omega_{e}$ of $\Omega$ (triangles/squares in $n_{d}=2$ or tetrahedra/cubes in $n_{d}=3$ ) such that $\bar{\Omega}=\overline{\bigcup_{e=1}^{n_{e l}} \Omega_{e}}$ (where $n_{e l}$ is the total number of elements). We denote by $h_{e}=h\left(\Omega_{e}\right)$ the diameter of element $e$ and $h=\max _{\Omega_{e} \in \mathcal{T}_{h}} h_{e}$. The boundary $\partial \Omega$ of $\Omega$ is denoted by $\Gamma, \Gamma_{e}$ is the set of all edges of element $\Omega_{e}$ and $\mathcal{E}$ is the collection of all edges of the mesh, including edges on the boundary $\Gamma$. We indicate with $\mathcal{E}^{o}=\mathcal{E} \backslash \Gamma$ the set of interior edges in the triangulation. Denoting by $\omega \subset \Omega$ a portion of $\Omega$ (e.g., an element domain $\Omega_{e}$ ), and by $\gamma$ a portion of $\mathcal{E}$, we define with

$$
(v, w)_{\omega}=\int_{\omega} v w \mathrm{~d} \omega \quad \text { and } \quad(\mathbf{v}, \mathbf{w})_{\omega}=\int_{\omega} \mathbf{v} \cdot \mathbf{w} \mathrm{d} \omega
$$

the $L^{2}(\omega)$ - and $\left(L^{2}(\omega)\right)^{n_{d}}$-inner products on the interior of $\omega$ and with

$$
\langle v, w\rangle_{\gamma}=\int_{\gamma} v w \mathrm{~d} \gamma \quad \text { and } \quad\langle\mathbf{v}, \mathbf{w}\rangle_{\gamma}=\int_{\gamma} \mathbf{v} \cdot \mathbf{w} \mathrm{d} \gamma
$$

boundary functionals on $\gamma$. The space $L^{2}(\mathcal{E})$ refers to the set of functions whose traces on the elements of $\mathcal{E}$ are square integrable. Let $\|v\|^{2}=(v, v)_{\omega}$, and let $W_{j}^{k}(\Omega)$ be the Sobolev space with norm

$$
\|v\|_{W_{j}^{k}(\Omega)}=\left(\sum_{\alpha \leq k}\left\|\mathbf{D}_{x}^{\alpha} v\right\|_{L^{j}(\Omega)}^{j}\right)^{1 / j}, 1 \leq j<\infty .
$$

For $j=2$, let $H^{k}(\Omega)=W_{2}^{k}(\Omega)$ and set $\|v\|_{W_{j}^{k}}=\|v\|_{k}$. Let $\mathbb{Q}^{k}\left(\Omega_{e}\right)$ (with $k \geq 1$ ) be the space of polynomials of at most order $k$, in the case of triangular/tetrahedral meshes, and the tensor product of polynomials of at most order $k$ in the case of quadrilateral and hexahedral elements. These polynomial spaces can be implemented using the bases of Lagrange or Legendre polynomials, and are instrumental in the definition of the space $V^{k}\left(\mathcal{T}_{h}\right)$, associated with a specific finite element method. For any function $w(\mathbf{x}, t) \in L^{2}\left([0, T] ; H^{k}(\Omega)\right)$ the finite element spatial approximation in $V^{k}\left(\mathcal{T}_{h}\right)$ is denoted by $W(\mathbf{x}, t)$ (using capital letters) that is $W(\mathbf{x}, t) \in C\left([0, T] ; V^{k}\left(\mathcal{T}_{h}\right)\right)$.

\subsection{Numerical discretizations for the flow equations}

In this section, we describe numerical approximations of the Darcy flow system of equations (19)- 20) (or, alternatively, (21) ) in the miscible displacement case. In order to simplify the discussion, we consider time-continuous, semi-discretized versions of the numerical methods.

\subsubsection{Finite difference and finite volume methods}

We give, next, a very brief introduction to finite difference methods for the flow problem. Readers interested in more details should refer to the cited contributions. The scope of our discussion here is to highlight the similarities (or equivalencies) between well-known finite difference schemes and variational methods, respectively.

Finite differences were the first and, initially, most widely employed methods in the oil and gas industry for the flow equations [175, 153]. These methods rely on an approximation to the strong form of the governing equations based 
on Taylor series. The order of accuracy is often times quantified through the truncation error of the series, although this approach leads to reliable estimates only on regular, uniformly spaced grids. There are two major classes of finite difference algorithms, depending on whether the solution degrees of freedom are node-centered (vertex-centered) or cell-centered (block-centered). Using appropriate quadrature rules, it is possible to show that, in their lowest-order incarnation, node-centered finite differences are equivalent to continuous piecewise linear Galerkin finite element approximations, whereas cell-centered finite differences are equivalent to the lowest order mixed finite element method of Raviart-Thomas type [153, 176, 177].

Considering standard finite difference notations in one dimension, $\left\{x_{i}\right\}_{0 \leq i \leq N}$ denotes the set of uniformly spaced grid points, where $x_{i}=i h, h=1 / N$ is the grid size and $N$ the number of cells, a given positive integer. Analogous definitions hold along the other dimensions. A function $p(x)$ can be approximated by $p_{i} \approx p\left(x_{i}\right)$ for all given points $i \in\{0, \cdots, N\}$. In two dimensions and neglecting gravity, the flow equation 21$\}$ at point $\left(x_{i}, y_{j}\right)$ can be approximated by

$$
\begin{aligned}
\partial_{t}\left(\tilde{\phi}_{i, j}\right)-\frac{1}{x_{i+1 / 2}-x_{i-1 / 2}}\left(\tilde{\boldsymbol{\kappa}}_{i+1 / 2, j} \frac{p_{i+1, j}-p_{i, j}}{x_{i+1}-x_{i}}\right. & \left.-\tilde{\boldsymbol{\kappa}}_{i-1 / 2, j} \frac{p_{i, j}-p_{i-1, j}}{x_{i}-x_{i-1}}\right) \\
& -\frac{1}{y_{j+1 / 2}-y_{j-1 / 2}}\left(\tilde{\boldsymbol{\kappa}}_{i, j+1 / 2} \frac{p_{i, j+1}-p_{i, j}}{y_{j+1}-y_{j}}-\tilde{\boldsymbol{\kappa}}_{i, j-1 / 2} \frac{p_{i, j}-p_{i, j-1}}{y_{j}-y_{j-1}}\right)=q_{i, j},
\end{aligned}
$$

for $1 \leq i \leq N-1$ and $1 \leq j \leq N-1$, with $x_{i+1 / 2}=\left(x_{i}+x_{i+1}\right) / 2$ and $y_{j+1 / 2}=\left(y_{j}+y_{j+1}\right) / 2$. According to our notation, in the case of node-centered finite differences, $\left(x_{i}, y_{j}\right)$ is the location of node $(i, j)$ and $\left(x_{i+1 / 2}, y_{j+1 / 2}\right)$ the location of the neighboring cell center. Vice versa, in a cell centered scheme, nodes are located at $\left(x_{i+1 / 2}, y_{j+1 / 2}\right)$ and cell centers at $\left(x_{i}, y_{j}\right)$. See Figure 3 for a graphical illustration.

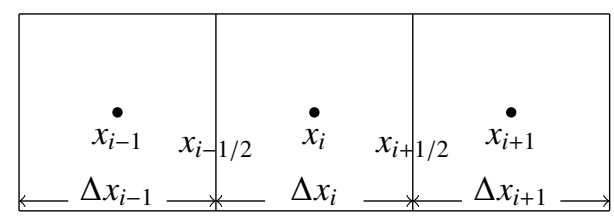

(a) Cell-centered grid

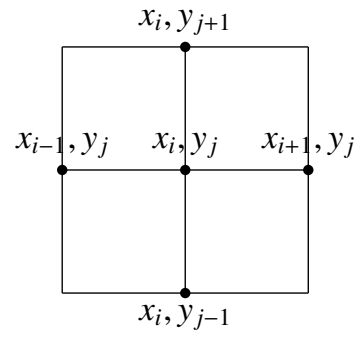

(b) Node-centered grid

Figure 3: Examples of nodes for cell-centered and mode-centered finite differences.

The convergence analysis with uniform meshes and smooth coefficients is identical for node-centered and cellcentered finite difference schemes, with the exception of the treatment of boundary conditions [178]. The proofs (which also involve a maximum principle) show quadratic convergence [179, 180, 181, 182]. For non-uniform meshes, the local truncation error is only first order for the node-centered scheme and $O(1)$ for the cell-centered scheme. However, $O(1)$ local truncation error does not necessarily preclude convergence, as established in [153, 183] with first-order estimates and [176, 184], with refined second-order estimates.

The engineering community initially favored cell-centered schemes because it is straightforward to prove their local conservation, while it became apparent only later that node-centered schemes can be proved locally conservative, resorting to the concept of a dual grid. Cell-centered schemes led to the development of numerical methods on general grid such as multipoint-flux mixed finite elements. Multipoint flux mixed finite elements can easily treat flow equations with distorted grids and rough full-tensor coefficients, and reduce to cell-centered pressure schemes under specific quadrature rules. Multipoint flux mixed finite elements overcome the complexity of two-point flux mixed finite element/finite volume methods, which are accurate only on Cartesian or very smooth grids [185, 186]. Node-centered schemes led to the development of generalized vertex-centered finite volume schemes.

By definition, finite volume schemes are locally conservative, since these discretizations originate from the integral conservation statements of the flow governing equations. In porous media simulations, finite volume methods were 
initially developed to treat realistic geological formations (on irregular grids) such as faults, pinch outs, and wells [187]. For a Poisson problem, the algebraic equations originating from a finite volume method on a uniform grid are equivalent to the ones obtained with a piecewise linear Galerkin finite element method. This equivalence motivated the strategy of computing fluxes for the Galerkin approximation on a dual grid, in order to prove local conservation.

Finite volumes and finite difference methods have been generalized to higher order, but a number of standing issues are object of intense research, first and foremost the lack of monotonicity [186, 188, 189, 190, 191, 192]. We will not further discuss these topics, since current extensions emphasize immiscible multiphase flow rather than miscible displacement.

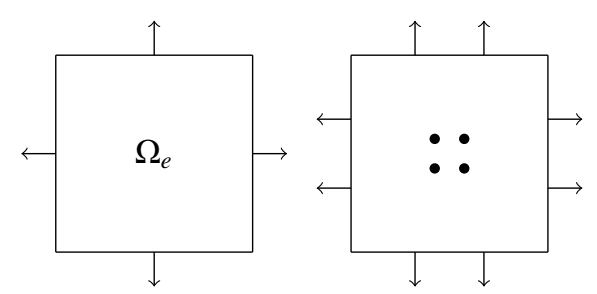

(a) $R T_{0}$ and $R T_{1}$

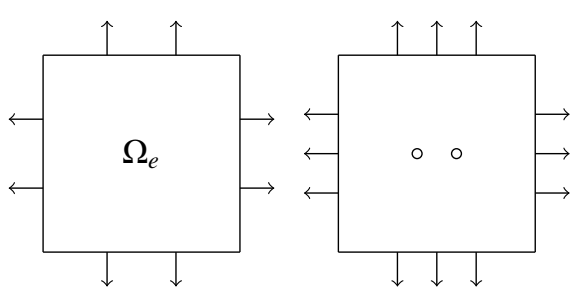

(b) $B D M_{1}$ and $B D M_{2}$

Figure 4: Degrees of freedom for two-dimensional mixed finite element spaces. Arrows indicate normal components of the velocity vector $\mathbf{u}$ and circles represent its moments on the element interior.

\subsubsection{Mixed finite element methods}

The basic idea of mixed methods for Darcy flow is use equations (19)- 20 ) to simultaneously approximate the pressure and velocity fields, rather than using equation (21) for the pressure and post-process the velocity. It is a consequence of standard results in approximation theory that one order of convergence is lost in passing from pressure to Darcy velocity by differentiation (the Darcy velocity is obtained from the pressure gradient, cfr. (20)). Additional complications may arise when the $\kappa=\mathbf{K} / \mu$ has abrupt variations (rough case, often encountered when the viscosity depends nonlinearly on the solute concentration) or becomes discontinuous (e.g., when faults produce discontinuities in the permeability distribution). In more realistic systems, viscosity and interphase mass transfer would also be functions of the pressure, adding to the overall complexity. Because of these considerations, it would be desirable to have procedures that approximate both pressure and velocity to the same order of accuracy, and this goal can be achieved by means of mixed methods.

To fix the ideas, let $\mathbf{U} \in C([0, T] ; \boldsymbol{V})$ and $P \in C([0, T] ; W)$ be the spatial finite element approximations to $\mathbf{u}$ and $p$, respectively. Here $V=H(\operatorname{div} ; \Omega)$ where $H(\operatorname{div} ; \Omega)=\left\{\mathbf{v} \in\left(L^{2}(\Omega)\right)^{2}: \nabla \cdot \mathbf{v} \in L^{2}(\Omega)\right\}$, and $W=L^{2}(\Omega)$. Integrating by parts and introducing the Lagrange multiplier $\Lambda \in V \cdot \mathbf{n}$ on $\Gamma_{p ; D}$, we obtain the variational form of $(8)$ :

Find $(\mathbf{U}, P) \in C([0, T] ; \boldsymbol{V} \times W)$ such that, $\forall \mathbf{v} \in C([0, T] ; \boldsymbol{V})$ and $\forall w \in C([0, T] ; W)$ :

$$
\begin{aligned}
\left(\tilde{\boldsymbol{\kappa}}^{-1} \tilde{\mathbf{U}}, \mathbf{v}\right)-(P, \nabla \cdot \mathbf{v}) & =\langle\Lambda \mathbf{v} \cdot \mathbf{n}\rangle_{\Gamma_{p ; D}}-(\tilde{\mathbf{g}}, \mathbf{v}), \\
\left(\partial_{t} \tilde{\Phi}, w\right)+(\nabla \cdot \tilde{\mathbf{U}}, w) & =(q, w), \\
\langle\tilde{\mathbf{U}} \cdot \mathbf{n}, \mathbf{v} \cdot \mathbf{n}\rangle_{\Gamma_{p ; N}} & =\left\langle\tilde{u}_{n}, \mathbf{v} \cdot \mathbf{n}\right\rangle_{\Gamma_{p ; N}} .
\end{aligned}
$$

The existence and uniqueness of the solution to 95 follows from arguments based on the inf-sup conditions [193, 194]. On triangular and rectangular meshes in two dimensions, and their tetrahedral and hexahedral counterparts in three dimensions, mixed finite element spaces satisfy $\nabla \cdot V_{h}=W_{h}$, and, as a consequence, the approximations to $p$ and $\nabla \cdot \mathbf{u}$ have the same order of accuracy. Well-known mixed finite elements spaces $\left(\boldsymbol{V}_{h} \subset \boldsymbol{V}\right)$ include the Raviart-Thomas-Nedelec (RTN) spaces [195, 196], Brezzi-Douglas-Marini (BDM) spaces [197], Brezzi-DouglasFortin-Marini (BDFM) spaces [198], Brezzi-Douglas-Duran-Fortin (BDDF) spaces [199], or Chen-Douglas (CD) spaces [200]. For example, the Raviart-Thomas (RT) finite element space of index $k$ on a rectangular element $\Omega_{e} \in \mathcal{T}_{h}$ is defined as

$$
\boldsymbol{V}_{R T}^{k}\left(\Omega_{e}\right)=\mathbb{Q}_{k+1, k} \times \mathbb{Q}_{k, k+1} \text { and } W_{R T}^{k}\left(\Omega_{e}\right)=\mathbb{Q}_{k, k},
$$


and the BDM space is defined as

$$
\boldsymbol{V}_{B D M}^{k}=\left(\mathbb{Q}_{k}\right)^{2} \oplus \operatorname{span}\left\{\operatorname{curl}\left(x^{k+1} y\right), \operatorname{curl}\left(x y^{k+1}\right)\right\} \text { and } W_{B D M}^{k}\left(\Omega_{e}\right)=\mathbb{Q}_{k-1} .
$$

For these spaces, we have of course that $\nabla \cdot V\left(\Omega_{e}\right)=W\left(\Omega_{e}\right)$, where $\nabla \cdot V\left(\Omega_{e}\right)$ indicates the space of functions obtained by applying the divergence to members of the space $V\left(\Omega_{e}\right)$. The Raviart-Thomas (RT) spaces of index $k \geq 0$ approximate $\mathbf{u}, p$, and $\nabla \cdot \mathbf{u}$ to the same order, $O\left(h^{k+1}\right)$, and are for this reason referred to as full approximation spaces in $H(\operatorname{div} ; \Omega)$, whereas BDM spaces of index $k \geq 1$ approximate $\mathbf{u}$ to the order $O\left(h^{k+1}\right)$, but $p$ and $\nabla \cdot \mathbf{u}$ only to order $O\left(h^{k}\right)$, and are therefore called reduced approximation spaces in $H(\operatorname{div} ; \Omega)$. The number of degrees of freedom for the RT and BDM spaces are, respectively, $\operatorname{dim}\left(\boldsymbol{V}_{R T}^{k}\left(\Omega_{e}\right) \times W_{R T}^{k}\left(\Omega_{e}\right)\right)=2(k+1)(k+2)$ and $\operatorname{dim}\left(V_{B D M}^{k}\left(\Omega_{e}\right) \times W_{B D M}^{k}\left(\Omega_{e}\right)\right)=$ $(k+1)(k+2)+2$, respectively (see Figure $4(\mathrm{a})-(\mathrm{b}))$.

\subsubsection{Continuous Galerkin methods}

Whenever low magnitudes of the dispersion tensor $\mathbf{D}$ and/or sharp fronts occur in the solution, finite difference approximations to miscible displacement flow problems can require very fine grids and may be very sensitive to to grid orientation. The latter circumstance can produce considerably different solutions for different orientations of the computational grids, as already discussed in Section 3.2. This motivated early developments and applications of variational methods [201, 202, 203, 204]. For example, one of the first variational methods in two spatial dimensions was developed in [203], in which cubic Hermite polynomials were used, and results of computations up to viscosity ratios of 100 were presented. Considering a finite-dimensional subspace $V$ of $H^{1}(\Omega)$, the variational form of a continuous Galerkin (CG) method can be presented as follows:

Find $P(\mathbf{x}, t) \in V$, such that, $\forall v \in C([0, T] ; V)$,

$$
\left(\partial_{t} \tilde{\Phi}, v\right)_{\Omega}+(\nabla(\tilde{\boldsymbol{\kappa}} \nabla P-\tilde{\mathbf{g}}), \nabla v)_{\Omega}+\langle P, v\rangle_{\Gamma_{p ; D}}=(q, v)_{\Omega}+\left\langle\tilde{u}_{n}, v\right\rangle_{\Gamma_{p ; N}}+\left\langle p_{D}, v\right\rangle_{\Gamma_{p ; D}},
$$

where Dirichlet boundary conditions are imposed weakly, in order to have a simpler general statement of global conservation.

In [155], the first a priori error estimates for CG methods applied to the coupled quasilinear system (19)-(20) were presented, for both semi- and fully-discretized-in-time equations. In particular, the analysis in [155] included a priori error estimates and emphasized that the approximations for concentration and pressure can lie in different subspaces of $H^{1}(\Omega)$. It was shown in particular that $L^{2}$-rates of convergence are optimal if the dispersion coefficient depends only upon molecular diffusion and if pressure and concentration lie in the same subspaces. These results were also extended to the discrete time case.

Extension of the analysis to sources and sinks as point singularities or Dirac measures was first presented in [205], in the case of unit mobility ratio. Due to the reduced smoothness, nonstandard analysis for the semi discrete CG procedures were used, and convergence rates of order lower than for nonsingular sources and sinks were obtained. Singularities arising from injection/production wells motivated the adoption of mesh-refinement techniques. In [206], an automated adaptive finite element procedure was developed, allowing different grids and time steps for pressure and concentration. In particular time-dependent mesh adaptation was applied to the concentration transport equations. This method included a decomposition of the solution, in which, in the early stages of the flooding process, an analytic asymptotic solution was used, while at later stages this asymptotic solution was blended with standard finite elements. This approach can then be considered as a precursor to generalized and extended finite elements. Several numerical examples of the five-spot flood problem were also presented in [206].

In [207], optimal (quadratic) order of convergence for semi-discrete piecewise linear element approximations was proven under weaker assumptions with respect to [205]. Extensions of these methods to slightly compressible flow was obtained in [38]. More recently, a mixed CG formulation has been proposed in [208].

In the past, criticism has been directed to CG on the lack of local conservation, but this statement is not completely correct, in the sense that CG methods are locally conservative [161], but the numerical fluxes that are conserved are nodal, in analogy to dual-grid finite volume methods. Hence, in a CG method, at every node, the sum of the numerical fluxes is conserved (in analogy with the nodal forces being balanced when CG methods are applied to mechanics problems). This statement of conservation, which is nodal rather than elemental, is less useful in preventing oscillations, since material properties in a subsurface porous medium are typically discontinuous at element interfaces. 
In summary, early CG works focused on modeling wells and grid orientation, most of the numerical examples considered homogeneous or piecewise constant permeabilities and mobility ratios in the range 10-100. Under these conditions, the unavoidable elemental mass conservation errors of CG elements were generally small and considered acceptable for engineering applications (recall that local conservation statements in CG elements involve a patch of elements, see Section 3.3 .

\subsubsection{Interior penalty Galerkin methods}

The symmetric interior penalty Galerkin (SIPG) method, initially proposed in [209, 210, 211], effectively represents one of the very early precursors of the discontinuous Galerkin (DG) method, and has become of widespread use for diffusion operators. The development of the SIPG formulation was motivated by the quest for a Galerkin method that could handle highly varying or discontinuous coefficients, singularities at wells, and could also provide a statement of local mass conservation analogous to finite volume methods. The SIPG successfully addresses all these requirements by means of a discontinuous approximation of the solution, and the introduction of a specifically designed variational formulation. Optimal $L^{2}$ convergence rates were proved in [210]. Like the DG methods that followed, the SIPG method can be adapted to general partial differential equations, and shows improved robustness with respect to CG methods in the case of abrupt changes in equation parameters and material properties. One disadvantage that the SIPG and DG methods share is the increase in a number of degrees of freedom, and ultimately computational cost, with respect to $\mathrm{CG}$ methods.

We introduce next some notation that for non-conforming and discontinuous finite element spaces. Consider a generalized edge of the finite element mesh, and denote by + and - the element on either side of it. In the case of SIPG and DG approximations, the discrete spaces are discontinuous across element interfaces, and it becomes useful to define the jump operator $\mathbb{[} \cdot \mathbb{\|}$ and average operator $\{[\cdot\}$ for discontinuous scalars and vector fields as follows:

$$
\begin{aligned}
& \llbracket v \rrbracket=v^{+} \mathbf{n}^{+}+v^{-} \mathbf{n}^{-}, \quad \llbracket \mathbf{v} \rrbracket=\mathbf{v}^{+} \cdot \mathbf{n}^{+}+\mathbf{v}^{-} \cdot \mathbf{n}^{-}, \\
& \{\{v\}\}=\frac{1}{2}\left(v^{+}+v^{-}\right), \quad\left\{\{\mathbf{v}\}=\frac{1}{2}\left(\mathbf{v}^{+}+\mathbf{v}^{-}\right) .\right.
\end{aligned}
$$

These definitions are very valuable when deriving global DG formulations, and in particular, are invariant with respect of any intrinsic orientation of the element interfaces. The following identity can also be verified [212], for a scalar $\alpha$ and a vector $\mathbf{v}$ :

$$
\llbracket \alpha \mathbf{v} \rrbracket=\{\{\alpha\}\} \llbracket \mathbf{v} \rrbracket+\llbracket \alpha \rrbracket \cdot\{\{\mathbf{v}\}\} .
$$

More generally, considering a scalar $\xi \in[0,1]$, and the convex-combinations

$$
\left\{\{v\}_{\xi}=\xi v^{+}+(1-\xi) v^{-}, \quad\left\{\{\mathbf{v}\}_{\xi}=\xi \mathbf{v}^{+}+(1-\xi) \mathbf{v}^{-},\right.\right.
$$

it is also possible to verify that

$$
\llbracket \alpha \mathbf{v} \rrbracket=\left\{\{v\}_{\xi} \llbracket \mathbf{v} \rrbracket+\llbracket \alpha \rrbracket \cdot\left\{\{\mathbf{v}\}_{1-\xi},\right.\right.
$$

which includes 100a as a special case when $\xi=1 / 2$. An interesting case to consider is also the one in which the superscript "_" indicates the upwind element (the one such that $\left\{\{\mathbf{u}\} \cdot \mathbf{n}^{-}>0\right.$ ), and the superscript "+" its downwind neighbor. In this case (102) becomes

$$
\llbracket \alpha \mathbf{v} \rrbracket=\alpha^{ \pm} \llbracket \mathbf{v} \rrbracket+\llbracket \alpha \rrbracket \cdot \mathbf{v}^{\mp} .
$$

Next, the following discontinuous functions spaces are introduced for the solution and test functions, respectively:

$$
\begin{aligned}
\mathcal{S} & =\left\{v \in L^{2}\left(\Omega_{e}\right):\left.v\right|_{\Omega_{e}} \in H^{k}\left(\Omega_{e}\right), \forall \Omega_{e} \in \mathcal{T}_{h}\right\}, \\
\mathcal{V} & =\left\{\mathbf{v} \in\left(L^{2}\left(\Omega_{e}\right)\right)^{n_{d}}:\left.\mathbf{v}\right|_{\Omega_{e}} \in\left(H^{k}\left(\Omega_{e}\right)\right)^{n_{d}}, \forall \Omega_{e} \in \mathcal{T}_{h}\right\} .
\end{aligned}
$$

$\mathcal{S}$ and $\mathcal{V}$ are spaces of functions whose restrictions to each element belong to the Sobolev spaces $H^{k}\left(\Omega_{e}\right)$ and $\left(H^{k}\left(\Omega_{e}\right)\right)^{n_{d}}$, respectively. $\mathcal{S}$ and $\mathcal{V}$ are often referred to as "broken spaces", because of their discontinuous nature. 
Their discrete counterparts are

$$
\begin{aligned}
\mathcal{S}_{h}^{k} & =\left\{v \in L^{2}\left(\Omega_{e}\right):\left.v\right|_{\Omega_{e}} \in \mathbb{Q}^{k}\left(\Omega_{e}\right), \forall \Omega_{e} \in \mathcal{T}_{h}\right\}, \\
\mathcal{V}_{h}^{k} & =\left\{\mathbf{v} \in\left(L^{2}\left(\Omega_{e}\right)\right)^{n_{d}}:\left.\mathbf{v}\right|_{\Omega_{e}} \in\left(\mathbb{Q}^{k}\left(\Omega_{e}\right)\right)^{n_{d}}, \forall \Omega_{e} \in \mathcal{T}_{h}\right\} .
\end{aligned}
$$

In order to make the notation more compact, we introduce a generalized source $\psi$, defined as

$$
\psi=q-\partial_{t} \tilde{\phi}
$$

so that 19$]-(20)$ reduce to

$$
\begin{aligned}
\nabla \cdot \tilde{\mathbf{u}} & =\psi \\
\tilde{\mathbf{u}} & =-\tilde{\boldsymbol{\kappa}}(\nabla P-\tilde{\mathbf{g}}) .
\end{aligned}
$$

Consider now a weak form of (107a)-(107b), in which integration-by-parts has been applied on every element. As before, $\tilde{\mathbf{U}}$ is the polynomial approximation to the velocity flux $\tilde{\mathbf{u}}$ and $P$ is the polynomial approximation to the pressure p. Then we seek for the solution to the discrete problem:

Find $P \in \mathcal{S}_{h}^{k}$ and $\tilde{\mathbf{U}} \in \mathcal{V}_{h}^{k}$ such that, $\forall w \in \mathcal{S}_{h}^{k}$ and $\forall \tau \in \mathcal{V}_{h}^{k}$,

$$
\begin{aligned}
& (w, \psi)_{\Omega_{e}}=-(\nabla w, \tilde{\mathbf{U}})_{\Omega_{e}}+\left\langle w, \hat{\mathbf{U}} \cdot \mathbf{n}_{e}\right\rangle_{\Gamma_{e} \backslash \Gamma}+\left\langle w, \hat{\tilde{\mathbf{U}}}_{\Gamma} \cdot \mathbf{n}_{e}\right\rangle_{\Gamma_{e} \cap \Gamma}, \\
& (\boldsymbol{\tau}, \tilde{\mathbf{U}})_{\Omega_{e}}=(\nabla \cdot(\tilde{\boldsymbol{\kappa}} \boldsymbol{\tau}), P)_{\Omega_{e}}+(\boldsymbol{\tau}, \tilde{\boldsymbol{\kappa}} \tilde{\mathbf{g}})_{\Omega_{e}}-\left\langle\mathbf{n}_{e} \cdot(\tilde{\boldsymbol{\kappa}} \boldsymbol{\tau}), \hat{P}\right\rangle_{\Gamma_{e} \backslash \Gamma}-\left\langle\mathbf{n}_{e} \cdot(\tilde{\boldsymbol{\kappa}} \boldsymbol{\tau}), \hat{P}_{\Gamma}\right\rangle_{\Gamma_{e} \cap \Gamma},
\end{aligned}
$$

where appropriate numerical fluxes $\hat{\tilde{\mathbf{U}}}$ and $\hat{P}$ have been introduced on the boundary of every element, to complete the specification of the computational strategy. The following choice of numerical fluxes leads to the SIPG method:

$$
\begin{aligned}
& \hat{\tilde{\mathbf{U}}}=-\{\tilde{\boldsymbol{\kappa}}(\nabla P-\tilde{\mathbf{g}})\}_{\xi}+\frac{\varepsilon(k)}{h_{e}^{\perp}}\{\tilde{\boldsymbol{\kappa}}\}_{\xi} \llbracket P \rrbracket, \\
& \hat{P}=\left\{\{P\}_{1-\xi},\right. \\
& \hat{\tilde{\mathbf{U}}}_{\Gamma}=\left\{\begin{array}{cc}
-\tilde{\boldsymbol{\kappa}}(\nabla P-\tilde{\mathbf{g}})+\frac{\varepsilon(k)}{h_{e}^{\perp}}\left(P-P_{D}\right) \tilde{\boldsymbol{\kappa}} \mathbf{n}, & \text { on } \Gamma_{P ; D}, \\
\tilde{u}_{n} \mathbf{n}, & \text { on } \Gamma_{P ; N},
\end{array}\right. \\
& \hat{P}_{\Gamma}=\left\{\begin{array}{cc}
P_{D}, & \text { on } \Gamma_{P ; D}, \\
P, & \text { on } \Gamma_{P ; N},
\end{array}\right.
\end{aligned}
$$

where $\varepsilon(k)$ is a numerical penalty parameter that may depend on the order of the polynomial approximation utilized, $h_{e}^{\perp}$ is a characteristic length scale, which can be defined on each edge/face of the grid as

$$
h_{e}^{\perp}= \begin{cases}\frac{\operatorname{meas}\left(\Omega_{e}^{+}\right)+\operatorname{meas}\left(\Omega_{e}^{-}\right)}{2 \operatorname{meas}\left(\gamma_{e}\right)}, & \text { if } \gamma_{e} \in \Gamma_{e} \backslash \Gamma, \\ \frac{\operatorname{meas}\left(\Omega_{e}\right)}{\operatorname{meas}\left(\gamma_{e}\right)}, & \text { if } \gamma_{e} \in \Gamma_{e} \cap \Gamma .\end{cases}
$$

Remark 9 (Local and global conservation). The SIPG mixed formulation is locally and globally conservative. Local conservation is proved by testing the variational form $108 \mathrm{~b}$ against a shape function equal to the unit constant on element $\Omega_{e}$ and zero everywhere else. This yields

$$
(1, \psi)_{\Omega_{e}}=\left\langle 1, \hat{\tilde{\mathbf{U}}} \cdot \mathbf{n}_{e}\right\rangle_{\Gamma_{e} \backslash \Gamma}+\left\langle 1, \hat{\tilde{\mathbf{U}}}_{\Gamma} \cdot \mathbf{n}_{e}\right\rangle_{\Gamma_{e} \cap \Gamma},
$$


which is a conservation statement for local element fluxes. Summing $(110)$ over all elements of the discretization, that is testing (108b against a shape function which is equal to unity over the entire computational domain $\Omega$, a global conservation statement is obtained, namely

$$
(1, \psi)_{\Omega}=\left\langle 1, \hat{\tilde{\mathbf{U}}}_{\Gamma} \cdot \mathbf{n}_{e}\right\rangle_{\Gamma}
$$

The Euler-Lagrange equations for the local mixed form are obtained integrating once more by parts:

$$
\begin{aligned}
& 0=(w, \nabla \cdot \tilde{\mathbf{U}}-\psi)_{\Omega_{e}}+\left\langle w,(\hat{\tilde{\mathbf{U}}}-\tilde{\mathbf{U}}) \cdot \mathbf{n}_{e}\right\rangle_{\Gamma_{e} \backslash \Gamma}+\left\langle w,\left(\hat{\tilde{\mathbf{U}}}_{\Gamma}-\tilde{\mathbf{U}}\right) \cdot \mathbf{n}_{e}\right\rangle_{\Gamma_{e} \cap \Gamma}, \\
& 0=(\boldsymbol{\tau}, \tilde{\mathbf{U}}+\tilde{\boldsymbol{\kappa}}(\nabla P-\tilde{\mathbf{g}}))_{\Omega_{e}}+\left\langle(\tilde{\boldsymbol{\kappa}} \boldsymbol{\tau}) \cdot \mathbf{n}_{e}, \hat{P}-P\right\rangle_{\Gamma_{e} \backslash \Gamma}+\left\langle(\tilde{\boldsymbol{\kappa}} \boldsymbol{\tau}) \cdot \mathbf{n}_{e}, \hat{P}_{\Gamma}-P\right\rangle_{\Gamma_{e} \cap \Gamma},
\end{aligned}
$$

Consider now the sum of $(112 \mathrm{~b})$ over all elements. Rewriting sums over elements as sums over the set $\mathcal{E}$ of the grid edges/faces, applying the flux definitions (109a)-(109d), recalling identity (102), and observing that, by definition, $\llbracket \hat{P} \rrbracket=0$, we obtain

$$
\begin{aligned}
(\boldsymbol{\tau}, \tilde{\mathbf{U}})_{\Omega} & =-(\boldsymbol{\tau}, \tilde{\boldsymbol{\kappa}}(\nabla P-\tilde{\mathbf{g}}))_{\Omega}-\langle 1, \llbracket \tilde{\boldsymbol{\kappa}} \boldsymbol{\tau}(\hat{P}-P) \rrbracket\rangle_{\mathcal{E}^{o}}-\left\langle\mathbf{n} \cdot(\tilde{\boldsymbol{\kappa}} \boldsymbol{\tau}), P_{D}-P\right\rangle_{\Gamma_{P ; D}} \\
& =-(\boldsymbol{\tau}, \tilde{\boldsymbol{\kappa}}(\nabla P-\tilde{\mathbf{g}}))_{\Omega}-\left\langle\{\tilde{\boldsymbol{\kappa}} \boldsymbol{\tau}\}_{\xi}, \llbracket \hat{P}-P \rrbracket\right\rangle_{\mathcal{E}^{o}}-\langle\llbracket \tilde{\boldsymbol{\kappa}} \boldsymbol{\tau} \rrbracket, \underbrace{\hat{P}-\left\{\{P\}_{1-\xi}\right.}_{=0}\rangle_{\mathcal{E}^{o}}-\left\langle\mathbf{n} \cdot(\tilde{\boldsymbol{\kappa}} \boldsymbol{\tau}), P_{D}-P\right\rangle_{\Gamma_{P ; D}} \\
& =-(\boldsymbol{\tau}, \tilde{\boldsymbol{\kappa}}(\nabla P-\tilde{\mathbf{g}}))_{\Omega}+\left\langle\{\tilde{\boldsymbol{\kappa}} \boldsymbol{\tau}\}_{\xi}, \llbracket P \rrbracket\right\rangle_{\mathcal{E}^{o}}+\left\langle\mathbf{n} \cdot(\tilde{\boldsymbol{\kappa}} \boldsymbol{\tau}), P-P_{D}\right\rangle_{\Gamma_{P ; D}},
\end{aligned}
$$

and, if we substitute $\tau=\nabla w$, we have

$$
(\nabla w, \tilde{\mathbf{U}})_{\Omega}=-(\nabla w, \tilde{\boldsymbol{\kappa}}(\nabla P-\tilde{\mathbf{g}}))_{\Omega}+\left\langle\{\tilde{\boldsymbol{\kappa}} \nabla w\}_{\xi}, \llbracket P \rrbracket\right\rangle_{\mathcal{E}^{o}}+\left\langle\mathbf{n} \cdot(\tilde{\boldsymbol{\kappa}} \nabla w), P-P_{D}\right\rangle_{\Gamma_{P ; D}} .
$$

Taking the sum of (108a) over all the elements of the computational domain yields

$$
\begin{aligned}
(\nabla w, \tilde{\mathbf{U}})_{\Omega}= & -(w, \psi)_{\Omega}+\left\langle w, \tilde{u}_{n}\right\rangle_{\Gamma_{P ; N}}+\left\langle\llbracket w \rrbracket,\left\{\{\tilde{\mathbf{U}}\}_{\xi}\right\rangle_{\mathcal{E}^{o}}+\left\langle\llbracket w \rrbracket,\left(\varepsilon(k) / h_{e}^{\perp}\right)\{\tilde{\boldsymbol{\kappa}}\}_{\xi} \llbracket P \rrbracket\right\rangle_{\mathcal{E}^{o}}\right. \\
& +\langle w, \tilde{\mathbf{U}} \cdot \mathbf{n}\rangle_{\Gamma_{P ; D}}+\left\langle w,\left(\varepsilon(k) / h_{e}^{\perp}\right)\left(P-P_{D}\right) \mathbf{n} \cdot(\tilde{\boldsymbol{\kappa}} \mathbf{n})\right\rangle_{\Gamma_{P ; D}} \\
= & -(w, \psi)_{\Omega}+\left\langle w, \tilde{u}_{n}\right\rangle_{\Gamma_{P ; N}}-\left\langle\llbracket w \rrbracket,\{\tilde{\boldsymbol{\kappa}}(\nabla P-\tilde{\mathbf{g}})\}_{\xi}\right\rangle_{\mathcal{E}^{o}}+\left\langle\llbracket w \rrbracket,\left(\varepsilon(k) / h_{e}^{\perp}\right)\left\{\{\tilde{\boldsymbol{\kappa}}\}_{\xi} \llbracket P \rrbracket\right\rangle_{\mathcal{E}^{o}}\right. \\
& -\langle w, \tilde{\boldsymbol{\kappa}}(\nabla P-\tilde{\mathbf{g}}) \cdot \mathbf{n}\rangle_{\Gamma_{P ; D}}+\left\langle w,\left(\varepsilon(k) / h_{e}^{\perp}\right)\left(P-P_{D}\right) \mathbf{n} \cdot(\tilde{\boldsymbol{\kappa}} \mathbf{n})\right\rangle_{\Gamma_{P ; D}} .
\end{aligned}
$$

Substituting now 114 into 115, we obtain the final primal form of the SIPG method:

Find $P \in \mathcal{S}_{h}^{k}$ such that, $\forall w \in \mathcal{S}_{h}^{k}$,

$$
\mathrm{B}[\tilde{\boldsymbol{\kappa}} ; \tilde{\mathbf{g}} ; \wp(P)](P, w)_{\xi}=\mathrm{L}[\tilde{\boldsymbol{\kappa}} ; \tilde{\mathbf{g}} ; \psi ; \wp(P)](w)_{\xi},
$$

with

$$
\begin{aligned}
\mathrm{B}[\tilde{\boldsymbol{\kappa}} ; \tilde{\mathbf{g}} ; \wp(P)](P, w)_{\xi}= & (\nabla w, \tilde{\boldsymbol{\kappa}} \nabla P)_{\Omega} \\
& -\left\langle\{\tilde{\boldsymbol{\kappa}} \nabla w\}_{\xi}, \llbracket P \rrbracket\right\rangle_{\mathcal{E}^{o}}-\left\langle\llbracket w \rrbracket,\left\{\{\tilde{\boldsymbol{\kappa}} \nabla P\}_{\xi}\right\rangle_{\mathcal{E}^{o}}-\langle\tilde{\boldsymbol{\kappa}} \nabla w \cdot \mathbf{n}, P\rangle_{\Gamma_{P ; D}}-\langle w, \tilde{\boldsymbol{\kappa}} \nabla P \cdot \mathbf{n}\rangle_{\Gamma_{P ; D}}\right. \\
& +\left\langle\llbracket w \rrbracket,\left(\varepsilon(k) / h_{e}^{\perp}\right)\left\{\{\tilde{\boldsymbol{\kappa}}\}_{\xi} \llbracket P \rrbracket\right\rangle_{\mathcal{E}^{o}}+\left\langle w,\left(\varepsilon(k) / h_{e}^{\perp}\right) P \mathbf{n} \cdot(\tilde{\boldsymbol{\kappa}} \mathbf{n})\right\rangle_{\Gamma_{P ; D}},\right. \\
\mathrm{L}[\tilde{\boldsymbol{\kappa}} ; \tilde{\mathbf{g}} ; \psi ; \wp(P)](w)_{\xi}= & (w, \psi)_{\Omega}+(\nabla w, \tilde{\boldsymbol{\kappa}} \tilde{\mathbf{g}})_{\Omega} \\
& -\left\langle\llbracket w \rrbracket,\{\tilde{\boldsymbol{\kappa}} \tilde{\mathbf{g}}\}_{\xi}\right\rangle_{\mathcal{E}^{o}}-\langle w, \tilde{\boldsymbol{\kappa}} \tilde{\mathbf{g}} \cdot \mathbf{n}\rangle_{\Gamma_{P ; D}}-\left\langle\tilde{\boldsymbol{\kappa}} \nabla w \cdot \mathbf{n}, P_{D}\right\rangle_{\Gamma_{P ; D}}-\left\langle w, \tilde{u}_{n}\right\rangle_{\Gamma_{P ; N}} \\
& +\left\langle w,\left(\varepsilon(k) / h_{e}^{\perp}\right) P_{D} \mathbf{n} \cdot(\tilde{\boldsymbol{\kappa}} \mathbf{n})\right\rangle_{\Gamma_{P ; D}} .
\end{aligned}
$$

In our notation, the terms inside square brackets are data, like, for example, the conductivity of the medium $\tilde{\boldsymbol{\kappa}}$ and the gravity force term $\tilde{\mathbf{g}}$. The term $\wp(P)$ represents the set of all parameters that are necessary to specify the problem for the pressure, that is, the boundaries $\Gamma_{P ; D}$ and $\Gamma_{P ; N}$, and corresponding boundary conditions $P_{D}$ and $\tilde{u}_{n}$, and the source term $\psi$. This compact notation will be very useful when considering DG variational formulations for the transport problem. Note that we can think of the term $\mathrm{B}[\tilde{\boldsymbol{\kappa}} ; \tilde{\mathbf{g}} ; \wp(P)](P, w)_{\xi}$ as an operator applied to the test function $w$, with appropriate arguments for $\tilde{\boldsymbol{\kappa}}, \tilde{\mathbf{g}}, \wp(P), P, w$, and $\xi$. This interpretation is again important when considering the variational formulation for the transport of species concentrations.

Remark 10. Most often, the choice $\xi=1 / 2$ is made. In what follows, whenever this occurs, the superscript $\xi$ is omitted from the linear and bilinear forms of the interior penalty method. 
4.1.5. Generalizations: symmetric, nonsymmetric, and incomplete interior penalty methods

A more general variational formulation can be obtained if the terms $\left\langle\{\tilde{\boldsymbol{\kappa}} \nabla w\}_{\xi}, \llbracket P \rrbracket\right\rangle_{\mathcal{E}^{o}},\langle\tilde{\boldsymbol{\kappa}} \nabla w \cdot \mathbf{n}, P\rangle_{\Gamma_{P: D}}$, and $\langle\tilde{\boldsymbol{\kappa}} \nabla w$. $\left.\mathbf{n}, P_{D}\right\rangle_{\Gamma_{P ; D}}$ are multiplied by a signed integer parameter $\theta$. Hence, the variational problem can be expressed as:

Find $P \in \mathcal{S}_{h}^{k}$ such that, $\forall w \in \mathcal{S}_{h}^{k}$,

$$
\mathrm{B}[\tilde{\boldsymbol{\kappa}} ; \tilde{\mathbf{g}} ; \wp(P)](P, w)_{\theta, \xi}=\mathrm{L}[\tilde{\boldsymbol{\kappa}} ; \tilde{\mathbf{g}} ; \psi ; \wp(P)](w)_{\theta, \xi},
$$

with

$$
\begin{aligned}
\mathrm{B}[\tilde{\boldsymbol{\kappa}} ; \tilde{\mathbf{g}} ; \wp(P)](P, w)_{\theta, \xi}= & (\nabla w, \tilde{\boldsymbol{\kappa}} \nabla P)_{\Omega} \\
& -\left\langle\llbracket w \rrbracket,\left\{\{\tilde{\boldsymbol{\kappa}} \nabla P\}_{\xi}\right\rangle_{\mathcal{E}^{o}}-\langle w, \tilde{\boldsymbol{\kappa}} \nabla P \cdot \mathbf{n}\rangle_{\Gamma_{P ; D}}\right. \\
& +\theta\left\langle\left\{\{\tilde{\boldsymbol{\kappa}} \nabla w\}_{\xi}, \llbracket P \rrbracket\right\rangle_{\mathcal{E}^{o}}+\theta\langle\mathbf{n} \cdot(\tilde{\boldsymbol{\kappa}} \nabla w), P\rangle_{\Gamma_{P ; D}}\right. \\
& +\left\langle\llbracket w \rrbracket,\left(\varepsilon(k) / h_{e}^{\perp}\right)\left\{\{\tilde{\boldsymbol{\kappa}}\}_{\xi} \llbracket P \rrbracket\right\rangle_{\mathcal{E}^{o}}+\left\langle w,\left(\varepsilon(k) / h_{e}^{\perp}\right) P \mathbf{n} \cdot(\tilde{\boldsymbol{\kappa}} \mathbf{n})\right\rangle_{\Gamma_{P ; D}},\right. \\
\mathrm{L}[\tilde{\boldsymbol{\kappa}} ; \tilde{\mathbf{g}} ; \psi ; \wp(P)](w)_{\theta, \xi}= & (\nabla w, \tilde{\boldsymbol{\kappa}} \tilde{\mathbf{g}})_{\Omega}+(w, \psi)_{\Omega} \\
& -\left\langle\llbracket w \rrbracket,\{\tilde{\boldsymbol{\kappa}} \tilde{\mathbf{g}}\}_{\xi}\right\rangle_{\mathcal{E}^{o}}-\langle w, \tilde{\boldsymbol{\kappa}} \tilde{\mathbf{g}} \cdot \mathbf{n}\rangle_{\Gamma_{P ; D}}+\theta\left\langle\tilde{\boldsymbol{\kappa}} \nabla w \cdot \mathbf{n}, P_{D}\right\rangle_{\Gamma_{P ; D}}-\left\langle w, \tilde{u}_{n}\right\rangle_{\Gamma_{P ; N}} \\
& +\left\langle w,\left(\varepsilon(k) / h_{e}^{\perp}\right) P_{D} \mathbf{n} \cdot(\tilde{\boldsymbol{\kappa}} \mathbf{n})\right\rangle_{\Gamma_{P ; D}} .
\end{aligned}
$$

If $\theta=-1$ the SIPG method already discussed is obtained, if $\theta=1$, a nonsymmetric interior penalty Galerkin (NIPG) method is derived, and letting $\theta=0$ leads to to the incomplete interior penalty Galerkin (IIPG) method. Optimal rates of convergence were established in [213] for the NIPG method, and in [214, 215] for IIPG method.

Remark 11. In what follows, if the parameter $\theta$ is omitted in the linear or bilinear operators of the variational form, it is implicitly assumed that $\theta=-1$, and that the SIPG formulation is considered.

\subsubsection{Upwind-biased interior penalty Galerkin methods}

A form of interior penalty (IP) methods of special interest is derived when $\xi$ is selected according to an upwind strategy with respect to a given velocity field, that is $\xi$ is set for every edge so that $\xi=1$ corresponds to the upwind side of the edge. An element is on the upwind or downwind side of an edge/face, depending on the direction of the velocity field $\tilde{\mathbf{U}}$ or $\mathbf{U}$. Namely, the element whose normal $\mathbf{n}_{e}$ is such that $\mathbf{U} \cdot \mathbf{n}_{e}>0$ is upwind of the edge (or face), while if $\mathbf{U} \cdot \mathbf{n}_{e} \leq 0$ the element is considered downwind of the edge. In this section, we indicate with a "-" the upwind side of an edge/face of the grid, and with "+" the downwind side. Then, the numerical fluxes (109a)-(109b) are replaced, respectively, by upwind-biased fluxes, that is:

$$
\begin{aligned}
\hat{\mathbf{U}} & =-(\tilde{\boldsymbol{\kappa}}(\nabla P-\tilde{\mathbf{g}}))^{-}+\frac{\varepsilon(k)}{h_{e}^{\perp}}(\tilde{\boldsymbol{\kappa}})^{-} \llbracket P \rrbracket, \\
\hat{P} & =P^{+} .
\end{aligned}
$$

Repeating the steps that lead to the SIPG formulation, 117a - 117c yield the following general upwind interior penalty variational formulation:

Find $P \in \mathcal{S}_{h}^{k}$ such that, $\forall w \in \mathcal{S}_{h}^{k}$,

$$
\mathrm{B}[\tilde{\boldsymbol{\kappa}} ; \tilde{\mathbf{g}} ; \wp(P)](P, w)_{U ; \theta, \xi}=\mathrm{L}[\tilde{\boldsymbol{\kappa}} ; \tilde{\mathbf{g}} ; \psi ; \wp(P)](w)_{U ; \theta, \xi},
$$

with 


$$
\begin{aligned}
\mathrm{B}[\tilde{\boldsymbol{\kappa}} ; \tilde{\mathbf{g}} ; \wp(P)](P, w)_{U ; \theta, \xi}= & (\nabla w, \tilde{\boldsymbol{\kappa}} \nabla P)_{\Omega} \\
& -\left\langle\llbracket w \rrbracket,(\tilde{\boldsymbol{\kappa}} \nabla P)^{-}\right\rangle_{\mathcal{E}^{o}}-\langle w, \tilde{\boldsymbol{\kappa}} \nabla P \cdot \mathbf{n}\rangle_{\Gamma_{P, D}} \\
& +\theta\left\langle(\tilde{\boldsymbol{\kappa}} \nabla w)^{-}, \llbracket I \rrbracket\right\rangle_{\mathcal{E}^{o}}+\theta\langle\mathbf{n} \cdot(\tilde{\boldsymbol{\kappa}} \nabla w), P\rangle_{\Gamma_{P, D}} \\
& +\left\langle\llbracket w \rrbracket,\left(\varepsilon(k) / h_{e}^{\perp}\right)(\tilde{\boldsymbol{\kappa}})^{-} \llbracket P \rrbracket \rrbracket\right\rangle_{\mathcal{E}^{o}}+\left\langle w,\left(\varepsilon(k) / h_{e}^{\perp}\right) P \mathbf{n} \cdot(\tilde{\boldsymbol{\kappa}} \mathbf{n})\right\rangle_{\Gamma_{p ; D}},
\end{aligned}
$$

$\mathrm{L}[\tilde{\boldsymbol{\kappa}} ; \tilde{\mathbf{g}} ; \psi ; \wp(P)](w)_{U ; \theta, \xi}=(\nabla w, \tilde{\mathbf{k}} \tilde{\mathbf{g}})_{\Omega}+(w, \psi)_{\Omega}$

$$
\begin{aligned}
& -\left\langle\llbracket w \rrbracket,(\tilde{\boldsymbol{\kappa}} \tilde{\mathbf{g}})^{-}\right\rangle_{\mathcal{E}^{o}}-\langle w, \tilde{\boldsymbol{\kappa}} \tilde{\mathbf{g}} \cdot \mathbf{n}\rangle_{\Gamma_{P ; D}}+\theta\left\langle\tilde{\boldsymbol{\kappa}} \nabla w \cdot \mathbf{n}, P_{D}\right\rangle_{\Gamma_{P ; D}}-\left\langle w, \tilde{u}_{n}\right\rangle_{\Gamma_{P ; N}} \\
& +\left\langle w,\left(\varepsilon(k) / h_{e}^{\perp}\right) P_{D} \mathbf{n} \cdot(\tilde{\boldsymbol{\kappa}} \mathbf{n})\right\rangle_{\Gamma_{P ; D}} .
\end{aligned}
$$

At first sight, it may not seem apparent why one would consider an upwind-biased variational formulation in the case of a parabolic or elliptic equation. However, in connnection to the discussion about the DSC (discrete sum compatibility) principle of Section 3.4. equations (119a- $1119 \mathrm{c}$ can be interpreted as the result of summing a set of upwind-biased transport equations of the type found in Section 4.2.4 Specifically, it will be shown in Section 4.2.4. to which we refer for further details, that (119a)-(119c) combined with (137) satisfy the DSC principle.

\subsubsection{Discontinuous Galerkin methods}

Over the last two decades, discontinuous Galerkin (DG) methods have gained considerable popularity. The variational structure of DG methods is very similar to the one of interior penalty methods. We will consider here only some of the many DG algorithms found in the literature. The survey article [212] contains a fairly complete review of DG methods for elliptic boundary value problems. In particular, we consider a method developed by the authors of [216] (the method is also described in detail in [212]). Discontinuous Galerkin (DG) formulations can be designed by modifying the definition of the numerical fluxes (109a)-(109d) developed for the SIPG method. In particular, consider the DG method proposed in [216], in which the SIPG numerical fluxes 109a-109d) are used, but now 109a and $(109 \mathrm{c})$ are replaced, respectively, by

$$
\begin{aligned}
\hat{\tilde{\mathbf{U}}} & =\{\tilde{\mathbf{U}}\}_{\xi}+\frac{\varepsilon(k)}{h_{e}^{\perp}}\{\tilde{\boldsymbol{\kappa}}\}_{\xi} \llbracket P \rrbracket, \\
\hat{\mathbf{U}}_{\Gamma} & = \begin{cases}\tilde{\mathbf{U}}+\frac{\varepsilon(k)}{h_{e}^{\perp}}\left(P-P_{D}\right) \tilde{\boldsymbol{\kappa}} \mathbf{n}, & \text { on } \Gamma_{P ; D}, \\
\tilde{u}_{n} \mathbf{n}, & \text { on } \Gamma_{P ; N} .\end{cases}
\end{aligned}
$$

A global primal formulation can be obtained with derivations similar to the SIPG method, but this time, because of the definition of the DG fluxes, an additional key ingredient needs to be introduced, the concept of global lifting operators $r$ and $r_{\Gamma p: D}$, defined as

$$
\begin{aligned}
(r(\mathbf{w}), \mathbf{v})_{\Omega} & =-\left\langle\mathbf{w},\left\{\{\mathbf{v}\}_{\xi}\right\rangle_{\mathcal{E}^{o}},\right. \\
\left(r_{\Gamma_{P ; D}}(\mathbf{w}), \mathbf{v}\right)_{\Omega} & =-\langle\mathbf{w}, \mathbf{v}\rangle_{\Gamma_{P ; D}} .
\end{aligned}
$$

The lifting operators allow to relate the velocity variable $\tilde{\mathbf{U}}$ to the pressure $P$, and finally obtain the variational form:

Find $P \in \mathcal{S}_{h}^{k}$ such that, $\forall w \in \mathcal{S}_{h}^{k}$,

$$
\mathrm{B}[\tilde{\boldsymbol{\kappa}} ; \tilde{\mathbf{g}} ; \wp(P)](P, w)_{D G}=\mathrm{L}[\tilde{\boldsymbol{\kappa}} ; \tilde{\mathbf{g}} ; \psi ; \wp(P)](w)_{D G},
$$

with

$$
\begin{aligned}
\mathrm{B}[\tilde{\boldsymbol{\kappa}} ; \tilde{\mathbf{g}} ; \wp(P)](P, w)_{D G} & =\mathrm{B}[\tilde{\boldsymbol{\kappa}} ; \tilde{\mathbf{g}} ; \wp(P)](w)_{U}+\left(r(\llbracket w \rrbracket)+r_{\Gamma_{P, D}}(w), \tilde{\boldsymbol{\kappa}}\left(r(\llbracket P \rrbracket)+r_{\Gamma_{P ; D}}(P)\right)\right)_{\Omega}, \\
\mathrm{L}[\tilde{\boldsymbol{\kappa}} ; \tilde{\mathbf{g}} ; \psi ; \wp(P)](w)_{D G} & =\mathrm{L}[\tilde{\boldsymbol{\kappa}} ; \tilde{\mathbf{g}} ; \psi ; \wp(P)](w)_{U}+\left(r(\llbracket w \rrbracket)+r_{\Gamma_{P, D}}(w), \tilde{\boldsymbol{\kappa}} r_{\Gamma_{P, D}}\left(P_{D}\right)\right)_{\Omega} .
\end{aligned}
$$

The previous equations are interesting in elucidating the nature of DG methods, which complement a SIPG formulation with residual terms built with nonlocal lifting operators. Hence, in a sense, it can be argued that the SIPG method is the basis and prototype of most DG methods. We would also like to mention a different approach [217, 218, 219] to stable DG computations based on constructing stabilizing residuals on element interiors, by means of variational multiscale framework [220]. An interesting more general work on this subject is [221], in which the various stabilizing mechanisms associated with DG discretizations are surveyed and analyzed. 


\subsubsection{Upwind-Biased Compact Discontinuous Galerkin method}

Similar to the case of the upwind-biased SIPG method described in Section 4.1.6, it is possible to bias upwind the DG method described in the previous section. We will describe next, instead, an upwind-biased version of a compact DG (or, CDG) method [222], proposed by Scovazzi, Huang and co-authors [158, 157]. This work has connections to the formulation presented in [216] and also to the local DG formulations (LDG) described in [223]. Consider the following definition of numerical fluxes:

$$
\begin{aligned}
\hat{\mathbf{U}} & =\tilde{\mathbf{U}}^{-}+\frac{\varepsilon(k)}{h_{e}^{\perp}} \tilde{\boldsymbol{\kappa}}^{-} \llbracket P \rrbracket, \\
\hat{P} & =P^{+}, \\
\hat{\mathbf{U}}_{\Gamma} & =\left\{\begin{array}{cc}
\tilde{\mathbf{U}}+\frac{\varepsilon(k)}{h_{e}^{\perp}}\left(P-P_{D}\right) \tilde{\boldsymbol{\kappa}} \mathbf{n}, & \text { on } \Gamma_{P ; D}, \\
\tilde{u}_{n} \mathbf{n}, & \text { on } \Gamma_{P ; N},
\end{array}\right. \\
\hat{P}_{\Gamma} & =\left\{\begin{array}{cc}
P_{D}, & \text { on } \Gamma_{P ; D}, \\
P, & \text { on } \Gamma_{P ; N} .
\end{array}\right.
\end{aligned}
$$

These fluxes are a variant of the CDG and LDG methods. The steps to derive a global formulation are similar to an upwind SIPG method. In this case, the local lifting operators $r^{s}$ and $r_{\Gamma_{P: D}}^{s}$ are defined as

$$
\begin{gathered}
\left(r^{s}(\mathbf{w}), \mathbf{v}\right)_{\Omega}=-\left\langle\mathbf{w}, \mathbf{v}^{-}\right\rangle_{s}, \quad s \in \mathcal{E}^{o}, \\
\left(r_{\Gamma_{P ; D}^{s}}^{s}(q), \mathbf{v}\right)_{\Omega}=-\langle q, \mathbf{v} \cdot \mathbf{n}\rangle_{s}, \quad s \in \Gamma_{P ; D},
\end{gathered}
$$

where $s$ is a "side" of the mesh skeleton, that is an edge in two dimensions or a face in three dimensions. The local lifting operators can also be used to define global lifting operators. Before this step, it is useful to define extended local lifting operators

$$
\begin{aligned}
\left(\bar{r}^{s}(\mathbf{w}), \mathbf{v}\right)_{\Omega} & = \begin{cases}-\left\langle\mathbf{w}, \mathbf{v}^{-}\right\rangle_{s}, & \text { if } s \in \mathcal{E}^{o}, \\
0, & \text { otherwise },\end{cases} \\
\left(\bar{r}_{\Gamma_{P: D}^{s}}^{s}(q), \mathbf{v}\right)_{\Omega} & = \begin{cases}-\langle q, \mathbf{v} \cdot \mathbf{n}\rangle_{s}, & \text { if } s \in \Gamma_{P ; D}, \\
0, & \text { otherwise }\end{cases}
\end{aligned}
$$

Then global lifting operators can be defined as

$$
\begin{aligned}
(r(\mathbf{w}), \mathbf{v})_{\Omega} & =\sum_{s \in \mathcal{E}}\left(\bar{r}^{s}(\mathbf{w}), \mathbf{v}\right)_{\Omega}=-\sum_{s \in \mathcal{E}^{o}}\left\langle\mathbf{w}, \mathbf{v}^{-}\right\rangle_{s}, \\
\left(r_{\Gamma_{p ; D}}^{s}(q), \mathbf{v}\right)_{\Omega} & =-\sum_{s \in \mathcal{E}}\left(\bar{r}_{\Gamma_{p ; D}^{s}}^{s}(q), \mathbf{v}\right)_{\Omega}=-\sum_{s \in \Gamma_{P ; D}}\langle q, \mathbf{v} \cdot \mathbf{n}\rangle_{s} .
\end{aligned}
$$

With this definition of the lifting operators, we obtain an upwind version of the compact discontinuous Galerkin (CDG, [222]) formulation, which we can term upwind CDG or U-CDG [158, 157]. While the CDG method is a variant of the LDG method, the U-CDG can also be interpreted as an upwind variant of the method proposed in [216], and for this reason, it does not fit the canonical definition of the LDG method [223, 212]. The global variational formulation of the U-CDG method is then denoted with the subscript $U C$, and can be formulated as

Find $P \in \mathcal{S}_{h}^{k}$ such that, $\forall w \in \mathcal{S}_{h}^{k}$,

$$
\mathrm{B}[\tilde{\boldsymbol{\kappa}} ; \tilde{\mathbf{g}} ; \wp(P)](P, w)_{U C}=\mathrm{L}[\tilde{\boldsymbol{\kappa}} ; \tilde{\mathbf{g}} ; \psi ; \wp(P)](w)_{U C},
$$

with 


$$
\begin{aligned}
& \mathrm{B}[\tilde{\boldsymbol{\kappa}} ; \tilde{\mathbf{g}} ; \wp(P)](P, w)_{U C}=\mathrm{B}[\tilde{\boldsymbol{\kappa}} ; \tilde{\mathbf{g}} ; \wp(P)](w)_{U}+\sum_{s \in \mathcal{E}}\left(\bar{r}^{s}(\llbracket w \rrbracket)+\bar{r}_{\Gamma_{P ; D}}^{s}(w), \tilde{\boldsymbol{\kappa}}\left(\bar{r}^{s}(\llbracket P \rrbracket)+\bar{r}_{\Gamma_{P ; D}}^{s}(P)\right)\right)_{\Omega}, \\
& \mathrm{L}[\tilde{\boldsymbol{\kappa}} ; \tilde{\mathbf{g}} ; \psi ; \wp(P)](w)_{U C}=\mathrm{L}[\tilde{\boldsymbol{\kappa}} ; \tilde{\mathbf{g}} ; \psi ; \wp(P)](w)_{U}+\sum_{s \in \mathcal{E}}\left(\bar{r}^{s}(\llbracket w \rrbracket)+\bar{r}_{\Gamma_{P ; D}}^{s}(w), \tilde{\boldsymbol{\kappa}} \bar{r}_{\Gamma_{P ; D}}^{s}\left(P_{D}\right)\right)_{\Omega} .
\end{aligned}
$$

Also in the case of the proposed discretization, the specific choice of an upwind bias of the numerical fluxes is motivated by the DSC principle described in Section 3.4, and already discussed in the context of interior penalty methods in Section 4.1.8. Also in the case of the proposed DG discretization, we refer the reader to Section 4.2.4 on the DG discretization of the transport equations for further discussion.

\subsubsection{Enriched Galerkin methods}

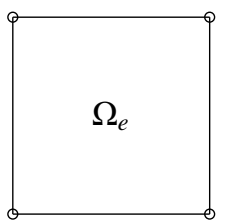

(a) $C G_{1}$

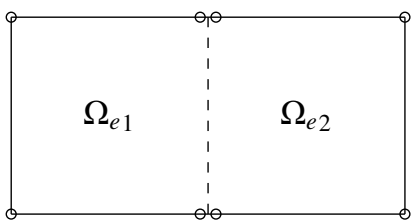

(b) $D G_{1}$

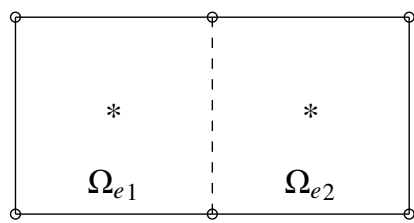

(c) $E G_{1}$

Figure 5: CG, DG and EG method approximations: a sketch of the degrees of freedom for a two-dimensional Cartesian grid $(\mathbb{Q})$.

In spite of the many advantages of IP and DG methods, one main disadvantage is their computational cost, due to the number of degrees of freedom and the conditioning of the ensuing algebraic system of equations. The Enriched Galerkin (EG) finite element method was recently proposed [224, 166] to overcome these limitations. The EG method is based on a CG formulation, in which however the solution is enriched by means of piecewise constant discontinuous functions. The EG method includes features of many of IP and DG methods, since it is locally and globally conservative, it can support non-matching grids and arbitrary order of local approximations, but it has considerably less degrees of freedom compared to IP or DG formulations. In addition, efficient solution algorithms for Darcy flow were developed in [166], and local grid adaptivity allowed to extend the EG method to realistic physical applications [174].These features enable the EG method to address the computational challenges discussed in Section 3 The EG approach is also intrinsically adaptive, since the enrichment is required only when satisfaction of elemental local conservation statements is deemed important.

The EG variational formulation is identical to the interior penalty formulation $(117 \mathrm{a})-(117 \mathrm{c})$ with $\xi=1 / 2$, but with a different finite element space approximation for the pressure, $P(\mathbf{x}, t) \in C\left([0, T] ; V_{h, k}^{\mathrm{EG}}\left(\mathcal{T}_{h}\right)\right)$. In order to define the space $V_{h, k}^{\mathrm{EG}}\left(\mathcal{T}_{h}\right)$, we first introduce the space of piecewise discontinuous polynomials of degree $k$ as

$$
M^{k}\left(\mathcal{T}_{h}\right):=\left\{\psi \in L^{2}(\Omega) \mid \psi_{\left.\right|_{K}} \in \mathbb{Q}_{k}\left(\Omega_{e}\right), \forall \Omega_{e} \in \mathcal{T}_{h}\right\}
$$

and let $M_{0}^{k}\left(\mathcal{T}_{h}\right)$ be the subspace of $M^{k}\left(\mathcal{T}_{h}\right)$ consisting of continuous piecewise polynomials, namely,

$$
M_{0}^{k}\left(\mathcal{T}_{h}\right)=M^{k}\left(\mathcal{T}_{h}\right) \cap C^{0}(\Omega)
$$

The enriched Galerkin finite element space, denoted by $V_{h, k}^{\mathrm{EG}}$ is defined as

$$
V_{h, k}^{\mathrm{EG}}\left(\mathcal{T}_{h}\right):=M_{0}^{k}\left(\mathcal{T}_{h}\right)+M^{0}\left(\mathcal{T}_{h}\right)
$$

where $k \geq 1$. Thus, starting from a CG finite element space, only piecewise constants are added for each $\Omega_{e}$ to obtain the final EG space. This approach introduces minimal additional cost for ensuring elemental local mass conservation statements, with numerical fluxes defined as in (109), as also shown in Figure 5. Optimal convergence rates for the EG method were proved in [166, 163] using harmonic averages, and conservation and accuracy of the EG approximation of the numerical fluxes in parabolic flow problems were discussed in [166]. Note that EG methods satisfy the DSC principle, provided that the IP or DG formulation they originate from conforms to it. See also Section 4.2.5 


\subsection{Numerical discretizations for the system of transport equations}

In this section, numerical methods for the solution of the species transport problem in porous media are discussed in detail. We make use of the notation and some results from Section 4.1. In particular, we analyze the various methods in terms of robustness, accuracy, and satisfaction of conservation and discrete compatibility principles. Our discussion will start with a brief introduction to numerical methods of finite difference and finite volume type, with the purpose of defining the context in which variational methods were later developed.

\subsubsection{Finite difference and finite volume methods}

In the industrial practice, it is widespread the use of cell-centered finite difference methods with upwind (or, upstream) weighting for the transport equations (18). Upwind weighting is necessary to ensure a maximum principle in the convection dominated limit. For example, in one dimension and for a two-species system where the solute mass fraction (or concentration) is denoted simply $c$, we have that (18) can be approximated by

$$
\frac{\partial}{\partial t}(\tilde{\phi} c)_{i}+\frac{1}{\Delta x_{i}}\left(\tilde{u}_{i+1 / 2} c_{i+1 / 2}-\tilde{\phi}_{i+1 / 2} \mathrm{D}\left(u_{i+1 / 2}\right) \frac{c_{i+1}-c_{i}}{\Delta x_{i+1 / 2}}\right)+\frac{1}{\Delta x_{i}}\left(\tilde{u}_{i-1 / 2} c_{i-1 / 2}-\tilde{\phi}_{i-1 / 2} \mathrm{D}\left(u_{i-1 / 2}\right) \frac{c_{i}-c_{i-1}}{\Delta x_{i-1 / 2}}\right)=q_{i},
$$

where the upwind weight is implemented in the definition of $c_{i+1 / 2}$, that is,

$$
c_{i+1 / 2}:= \begin{cases}c_{i}, & \text { if } \tilde{u}_{i+1 / 2}>0, \\ c_{i+1}, & \text { otherwise }\end{cases}
$$

and $\tilde{u}$ and $\mathrm{D}$ are the one-dimensional versions of $\tilde{\mathbf{u}}$ and $\mathbf{D}$. This scheme is conservative and stable, but only first-order accurate. Difference schemes that are not locally conservative may induce spurious oscillations. Schemes alternative to (129) have been proposed in the literature, to address the stability and accuracy issues, as well as robustness questions in terms of sensitivity to grid orientation, existence of a discrete maximum principle, etc. Among them, we mention monotone or TVD (Total Variation Diminishing) finite volume/difference schemes, and Godunov, LaxFriedrichs and Engquist-Osher schemes. These approaches are based on the use of flux/slope limiters that enforce an upper bound for the fluxes. Essentially non-oscillatory (ENO) and weighted essentially non-oscillatory (WENO) schemes were also introduced [225], and rely on specific polynomial reconstructions that increase the order of accuracy of discretizations while preserving robustness and stability. In parallel with these developments, we also mention the work of Godunov [226] on numerical methods based on Riemann solvers. In the case of an advection problem, the Godunov scheme collapses to upwinding. The original method proposed in [226] was only first-order accurate and strongly dissipative, and Godunov also presented a theorem stating that monotone linear schemes can only be firstorder. Nonlinear schemes, however, can be higher-order and monotone, and a second-order version of the Godunov scheme was proposed in [227]. In multiple dimensions additional complexities may arise due to the sensitivity of the results to grid orientation. This is particularly true for methods that are based on splitting along each Cartesian direction the multidimensional discrete gradient operators in the equations. To obviate to this problem, in[156, 228] a new, "unsplit" Godunov scheme was proposed. The literature on finite difference and finite volume methods for porous media flow is vast, and here we restrict our attention to (few of) the main contributions. A much greater number of numerical methods has been applied to the fields of aerospace and mechanical engineering, and geophysical/atmospheric sciences.

\subsubsection{The modified method of characteristics}

The modified method of characteristics (MMC) was introduced in [229] for the solution of the species transport equations in miscible displacement flows, as a way to overcome the limitations of small time steps and dependence of the results on grid orientation (particularly in the case of adverse mobility ratios). The MMC can be categorized among semi-Lagrangian methods, in which the Lagrangian time derivative is used to model convection, and regular finite difference or finite element discretizations are used to treat the diffusion and dispersion operators in the governing equations. The MMC has also some added benefits, in that it minimizes the overshoots that typically affect finite element methods near sharp fluid interfaces, has very low truncation error and for this reason allows relatively large stable time-steps without loss of accuracy. We briefly describe the method in the most general setting and without discretizing in space the equations, for the case, again, of a two-species transport system, where $c$ is the solute mass 
fraction. Observing that the mass flow equation (19) can be simplified from the transport equation (18), we have that these two yield:

$$
\tilde{\phi} \frac{\partial c}{\partial t}+\tilde{\mathbf{u}} \cdot \nabla c-\nabla \cdot(\tilde{\phi} \mathbf{D} \nabla c)=\hat{q}
$$

where $\hat{q}=q_{c}-c q$ with $q_{c}$ the source of the solute and $q$ the source of the flow. Then we transform 130 into the form

$$
\tilde{\psi}(x, t, \tilde{\mathbf{u}}) \frac{\partial c}{\partial \tau}-\nabla \cdot(\tilde{\phi} \mathbf{D} \nabla c)=\hat{q}
$$

where

$$
\begin{aligned}
\frac{\partial}{\partial \tau} & =\tilde{\psi}^{-1}\left(\tilde{\phi} \frac{\partial}{\partial t}+\tilde{\mathbf{u}} \cdot \nabla\right) \\
\tilde{\psi}(\mathbf{x}, t, \tilde{\mathbf{u}}) & =\left(\tilde{\phi}(\mathbf{x})^{2}+|\tilde{\mathbf{u}}(\mathbf{x}, t)|^{2}\right)^{1 / 2}
\end{aligned}
$$

In conclusion, 130 can be recast as

$$
\left(\tilde{\phi}(\mathbf{x})^{2}+|\tilde{\mathbf{u}}(\mathbf{x}, t)|^{2}\right)^{1 / 2} \frac{\partial c}{\partial \tau}-\nabla \cdot(\tilde{\phi} \mathbf{D} \nabla c)=\hat{q}
$$

Both finite differences or finite elements can be used to discretize the above equation along the characteristic flow direction $\tau=\tau(x, \tilde{\mathbf{u}})$. For example, the backward difference approximation of the derivative along the characteristic (or Lagrangian derivative) yields

$$
\frac{\partial}{\partial \tau} c\left(\mathbf{x}, t^{n+1}\right)=\frac{1}{\tilde{\psi}(\mathbf{x}, t, \tilde{\mathbf{u}}) \delta t}\left(c\left(\mathbf{x}, t^{n+1}\right)-c\left(\mathbf{x}-\frac{\tilde{\mathbf{u}}^{\star}(\mathbf{x})}{\tilde{\phi}} \delta t, t^{n}\right)\right),
$$

where $\tilde{\mathbf{u}}^{\star}$ is an approximate average velocity of a flux particle reaching $\mathbf{x}$ at time $t^{n+1}$.

The finite difference version of the method was formulated with convergence analyses in [229]. Early numerical simulations for miscible displacement flow in different well configurations were illustrated in [230, 231, 232]. Computations including gravity and heterogeneity effects were performed in [233, 234], where it was shown that the MMC can simulate adverse mobility ratio accurately with minimal grid orientation effects, reduced numerical dispersion, minimal overshoot at advancing fronts, and very low conservation errors.

One of the first numerical analyses for the coupling of the MMC with finite element or finite difference algorithms for the advection-diffusion equation was demonstrated in [235, 236]. Error estimates were presented for various finite difference schemes and optimal $L^{2}$ - and $H^{1}$-convergence estimates were derived for finite element methods. In [237], these results were improved by proving optimal convergence with weaker norm assumptions, and requiring the tensor of diffusion coefficients to be only positive semidefinite. The MMC for the transport problem was combined with mixed finite elements for the flow problem in [238], where difficulties were observed when highly heterogeneous permeabilities are encountered. An improved version of the method of [238] was formulated for two-dimensional flows in [239].

\subsubsection{Continuous Galerkin methods}

CG methods have been applied less often to porous media flow problems. Part of the reason can be found in the limitations of $\mathrm{CG}$ algorithms in solving the advective diffusive systems of equation peculiar to subsurface applications, which involve heterogeneous, anisotropic, rough coefficients. We already mentioned in Section 4.1.3 that CG methods have intrinsic local conservation statements for patches of elements around a node of the discretization, and for this reason these are less useful (See also Section 3.3). This is also true in the case of transport problem, which are typically exacerbated by heterogeneities in the porous medium.

For example, when permeabilities (and, more generally conductivities or mobilities) change abruptly in a porous medium, preferential channels of advection develop and, as a result, discontinuous profiles of concentrations (or saturations in the immiscible case) can form naturally along the cross-stream direction, as convection dominates. CG methods can only offer continuous approximations to the correct solution and lack a discrete maximum principle [240]. 
Consequently, CG discretizations are prone to the Gibbs phenomenon (overshoots and undershoots near sharp gradients). Various strategies are possible to reduce this inconvenience, such as the addition of cross-wind artificial diffusion, or general discontinuity capturing operators. As a consequence, however, the results of CG formulations will typically be overly dissipative with respect to discretizations that allow for discontinuous approximations.

In the available literature, the discretization of transport for miscible displacement was treated in [201, 202, 203, 204, 155, 205, 206] as already discussed in Section 4.1.3. These early numerical approaches were based on smooth $C^{1}$ spaces, which were however rather diffusive near sharp fronts. These early experiments motivated the development of IP methods, later on. More recently, in [241, 242, 243, 244], the Streamline Upwind Petrov Galerkin (SUPG) method [245] was adapted to miscible displacement simulations. In these works, the pressure equation is solved with a CG method and the velocity field is computed by way of post-processing, in order to recover the required accuracy.

\subsubsection{Interior penalty and upwind biased discontinuous Galerkin approximations}

As already mentioned, the dissipative nature of CG methods near sharp solution gradients and interfaces motivated the development of interior penalty and discontinuous Galerkin methods. The literature is vast, already in the field of numerical methods for porous media flows. In the case of the transport equations, the dependency of the dispersion tensor $\mathbf{D}$ on the velocity $\mathbf{u}$ may be particularly insidious, and IP and DG methods provided improved solutions in this case. We describe next some examples, and point the reader to some references for more details and discussions of methods not included here for the sake of brevity.

One important aspect is that the discretization may slightly differ depending on whether or not the discrete flow velocity $\mathbf{U}$ (or, analogously, $\tilde{\mathbf{U}}$ ) is explicitly represented in computations. This choice is fundamentally connected to the way the species transport equations are coupled to the flow equation, and to whether or not the flow and transport equations are solved with the same (or similar) discretizations. For example, if a mixed finite element method is used for the flow problem, then $\mathbf{U}$ (or, $\tilde{\mathbf{U}}$ ) is part of the solution, and is available discretely as a field. This may not be the case if a primal DG formulation is used to discretize the flow equation. For the case in which $\mathbf{U}$ is carried as a discretized field, the discretization takes the form:

$$
\left(w, \partial_{t}\left(\tilde{\phi} C_{i}\right)\right)_{\Omega}+\mathrm{B}[\tilde{\mathbf{U}}]\left(C_{i}, w\right)_{U}^{a d v}+\mathrm{B}\left[(\tilde{\phi} \mathbf{D}) ; \mathbf{0} ; \wp\left(C_{i}\right)\right]\left(C_{i}, w\right)_{*}=\mathrm{L}[\tilde{\mathbf{U}}](w)_{U}^{a d v}+\mathrm{L}\left[(\tilde{\phi} \mathbf{D}) ; \mathbf{0} ; \psi ; \wp\left(C_{i}\right)\right](w)_{*},
$$

where

$$
\begin{aligned}
\mathrm{B}[\tilde{\mathbf{U}}]\left(C_{i}, w\right)_{U}^{a d v} & =-\left(C_{i},(\tilde{\mathbf{U}} \cdot \nabla w)\right)_{\Omega}+\left\langle\left\{\tilde{\mathbf{U}} C_{i}\right\}_{U}, \llbracket w \rrbracket\right\rangle+\left\langle C_{i} \tilde{\mathbf{U}} \cdot \mathbf{n}, w\right\rangle_{\Gamma_{o u t}}, \\
\mathrm{~L}[\tilde{\mathbf{U}}](w)_{U}^{a d v} & =-\left\langle c_{B} \tilde{\mathbf{U}} \cdot \mathbf{n}, w\right\rangle_{\Gamma_{\text {in }}},
\end{aligned}
$$

and $\left\{\tilde{\mathbf{U}} C_{i}\right\}_{U}$ denotes the upwind flux given by

$$
\left\{\tilde{\mathbf{U}} C_{i}\right\}_{U}:=\left\{\begin{array}{cc}
\tilde{\mathbf{U}} C_{i}^{+} & \text {if } \tilde{\mathbf{U}} \cdot \mathbf{n}^{+}>0, \\
\tilde{\mathbf{U}} C_{i}^{-} & \text {if } \tilde{\mathbf{U}} \cdot \mathbf{n}^{+} \leq 0,
\end{array}\right.
$$

which, as shown in [246, 247], is equivalent to

$$
\left\{\{\tilde{\mathbf{U} C}\}_{U}=\left\{\{\tilde{\mathbf{U}} C\}_{\xi=1 / 2}+\frac{|\tilde{\mathbf{U}} \cdot \mathbf{n}|}{2} \llbracket C \rrbracket .\right.\right.
$$

In the term $\mathrm{B}\left[(\tilde{\phi} \mathbf{D}) ; \mathbf{0} ; \wp\left(C_{i}\right)\right]\left(C_{i}, w\right)_{*}$, the symbol $*$ can indicate an IP method, a LDG method, or UCDG method.

For IP methods, the authors of [248] demonstrated optimal convergence for transport system with DG-SIPG and DG-NIPG methods, and optimal convergence, also for negative norms in space (i.e., $H^{-1}(\Omega)$ ) was established in [214, 215]. The possible IP formulations include the SIPG method already discussed $(\theta=-1)$, a non-symmetric interior penalty Galerkin (NIPG, $\theta=1$ ), and incomplete interior penalty Galerkin method (IIPG, $\theta=0$ ). For the coupled flow and transport, the choice of pairs $\left(\theta_{p}, \theta_{c}\right)$ can be shown to be crucial for discrete sum compatibility [162, 163], where $\theta_{p}$ is used in the flow equation, and $\theta_{c}$ is used in the transport equations.

In the case a DG discretization is used, upwind-bias of the advective linear and bilinear operators $\mathrm{L}[\tilde{\mathbf{U}}](w)_{U}^{a d v}$ and $\mathrm{B}[\tilde{\mathbf{U}}]\left(C_{i}, w\right)_{U}^{a d v}$ is necessary for stability, but many different options can be selected for the diffusive operators $\mathrm{L}\left[(\tilde{\phi} \mathbf{D}) ; \mathbf{0} ; \psi ; \wp\left(C_{i}\right)\right](w)_{*}$ and $\mathrm{B}\left[(\tilde{\phi} \mathbf{D}) ; \mathbf{0} ; \wp\left(C_{i}\right)\right]\left(C_{i}, w\right)_{*}$, without precluding stability. For example, an LDG or CDG 
can be used, two other variants are also very interesting, namely, the symmetric DG method [216] described in Section 4.1.7 with $\xi=1 / 2$, which was implemented in [249, 250] for gravity-driven flows, or the upwind DG discretization of [158, 157] described in Section 4.1.8

If $\mathbf{U}$ (or, $\tilde{\mathbf{U}}$ ) is not carried as a variable, equation $(18)$ is replaced by

$$
\partial_{t}\left(\tilde{\phi} c_{i}\right)-\nabla \cdot\left(c_{i} \boldsymbol{\kappa}(\nabla p-\tilde{\mathbf{g}})+\tilde{\phi} \mathbf{D} \nabla c_{i}\right)=q_{i},
$$

for $i=1,2, \ldots, N_{c}$, and the IP and DG discretizations developed for the flow problem can be reused also for the transport problem, with appropriate modifications. In particular, the transport problem contains a Laplace-type term for the pressure $P$ in conjunction with a Laplace-type term for the concentration $c_{i}$. In [158], an upwind interior penalty and a upwind compact discontinuous Galerkin formulations were proposed for both terms:

$$
\begin{aligned}
& \text { Find } C_{1}, C_{2}, \ldots, C_{N_{c}-1}, p \in \mathcal{S}_{h}^{k} \text { such that, } \forall w \in \mathcal{S}_{h}^{k}, \\
& \qquad \begin{aligned}
\left(w, \partial_{t}\left(\tilde{\phi} C_{i}\right)\right)_{\Omega}+\mathrm{B}\left[\left(C_{i} \tilde{\boldsymbol{\kappa}}\right) ; \tilde{\mathbf{g}} ; \wp(P)\right](P, w)_{*}+\mathrm{B}\left[(\tilde{\phi} \mathbf{D}) ; \mathbf{0} ; \wp\left(C_{i}\right)\right]\left(C_{i}, w\right)_{*} \\
\quad=\mathrm{L}\left[\left(C_{i} \tilde{\boldsymbol{\kappa}}\right) ; \tilde{\mathbf{g}} ; q_{i} ; \wp(P)\right](w)_{*}+\mathrm{L}\left[(\tilde{\phi} \mathbf{D}) ; \mathbf{0} ; \psi ; \wp\left(C_{i}\right)\right](w)_{*},
\end{aligned}
\end{aligned}
$$

where $\wp\left(C_{i}\right)$ represents the set of all parameters that are necessary to specify the problem for the concentration $C_{i}$, that is, the boundaries $\Gamma_{C_{i} ; D}$ and $\Gamma_{C_{i} ; N}$, and corresponding boundary conditions $C_{i ; D}$ and $h_{C_{i}}$, the source/sink term $q_{i}$ and initial condition $C_{i ; 0}$. Note also that the superscript $*$ may be replaced by $U$ or $U C$, corresponding to an upwindbiased SIPG method, or the upwind-biased UC-DG method. Due to the nature of transport, upwind discretizations are necessary for stability. Consider now the sum of equations (137) over all the species $i$ of the transport system. Recalling that $\sum_{i}^{N_{c}} c_{i}=1$, it is then relatively easy to prove that the sum of (137) is given by

$$
\left(w, \partial_{t} \tilde{\phi}\right)_{\Omega}+\mathrm{B}[\tilde{\boldsymbol{\kappa}} ; \tilde{\mathbf{g}} ; \wp(P)](P, w)_{*}=\mathrm{L}[\tilde{\boldsymbol{\kappa}} ; \tilde{\mathbf{g}} ; \psi ; \wp(P)](w)_{*},
$$

which exactly corresponds to the upwind biased IP or DG discretizations for the flow equation described in Section 4.1.6 and 4.1.8, namely (119a) or (125a). In [158, 157], $N_{c}-1$ equations (137) for the transport of species were coupled with the flow equation (138), using a fully coupled approach with Newton solves as described in Section 3.1.2. The resulting coupled systems satisfies the DSC principle by construction. It becomes a little more clear at this point, the reason for introducing upwind-biased discretizations of Laplace-type operators for the flow equations in Section 4.1.6 and 4.1.8

\subsubsection{Enriched Galerkin methods}

The EG method can also be used when discretizing the transport system. In this case, we have that $C(\mathbf{x}, t) \in$ $C\left([0, \mathbb{T}] ; V_{h, k}^{\mathrm{EG}}\left(\mathcal{T}_{h}\right)\right)$, where $V_{h, k}^{\mathrm{EG}}\left(\mathcal{T}_{h}\right)$ is given in $[128)$. The EG method for transport is based on the IP formulation outlined in (134), and utilizes the upwind bias of the fluxes (135). Stability and optimal error convergence analyses are presented in [163], where it also shown which choices of EG base IP variational formulation conform to the DSC principle.

It is clear that the EG discretizations inherit the ability to satisfy the DSC principle directly from the base IP or DG methods they derive from. We will not comment further on this point, already discussed at length in the previous sections.

In [174], with the purpose of avoiding spurious oscillations occurring in the convection dominated case, an entropy viscosity residual stabilization operator is utilized. We refer to [251, 252] for additional details, suffices to say that the local residual of an entropy equation is used to construct a nonlinear numerical dissipation operator, to be added to the EG weak form of the transport problem. This technique is independent of mesh and order of approximation. Moreover, the entropy residual is employed as an error estimator for dynamic adaptive mesh refinement [174].

\section{Applications}

In this section we present a number of applications of numerical methods for miscible displacement and transport. The list of problems considered is certainly not exhaustive, but it can give a general idea of the broad applicability of miscible displacement flows and also the computational challenges to be addressed. 


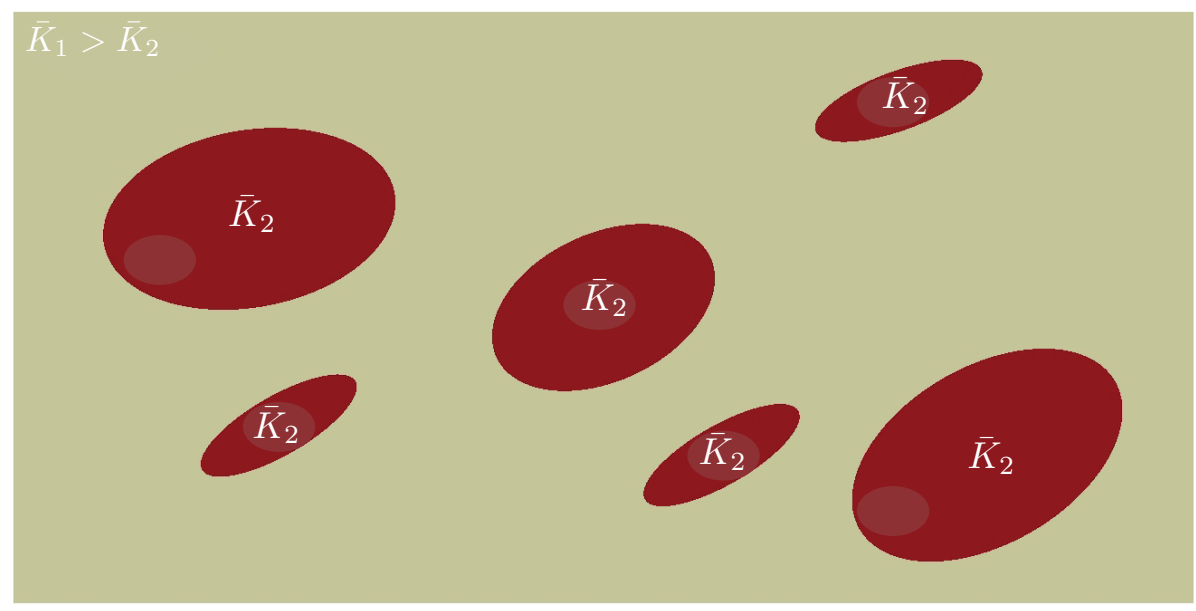

(a) Sketch of the complex geometry domain and distribution of permeability in the general case.

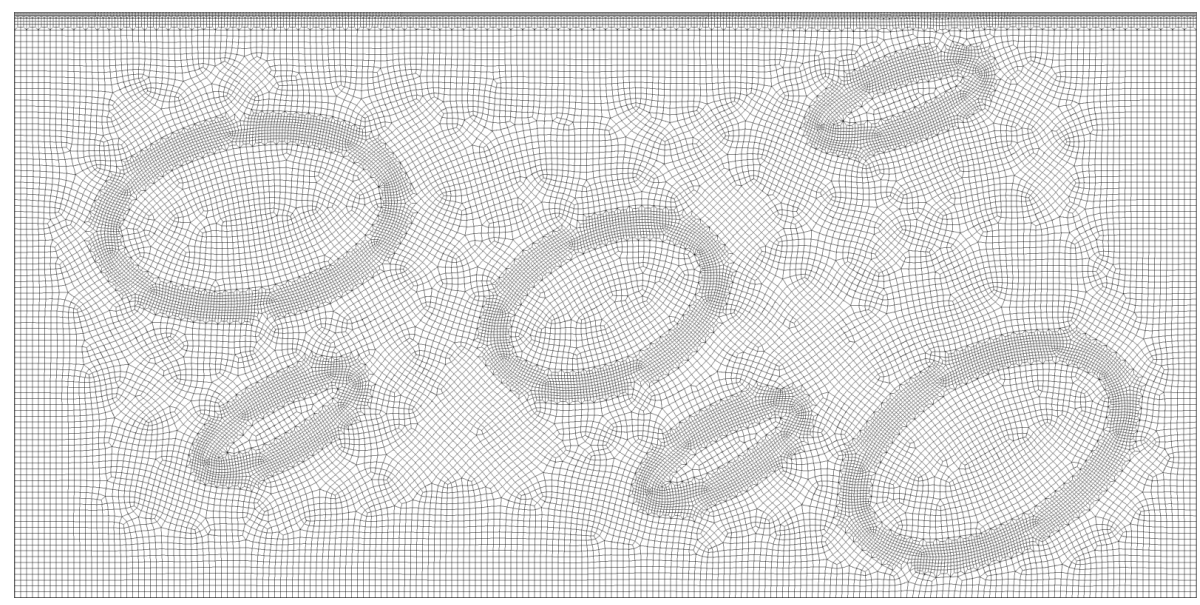

(b) Mesh detail in the case $\bar{K}_{2}=\bar{K}_{1} / 10$.

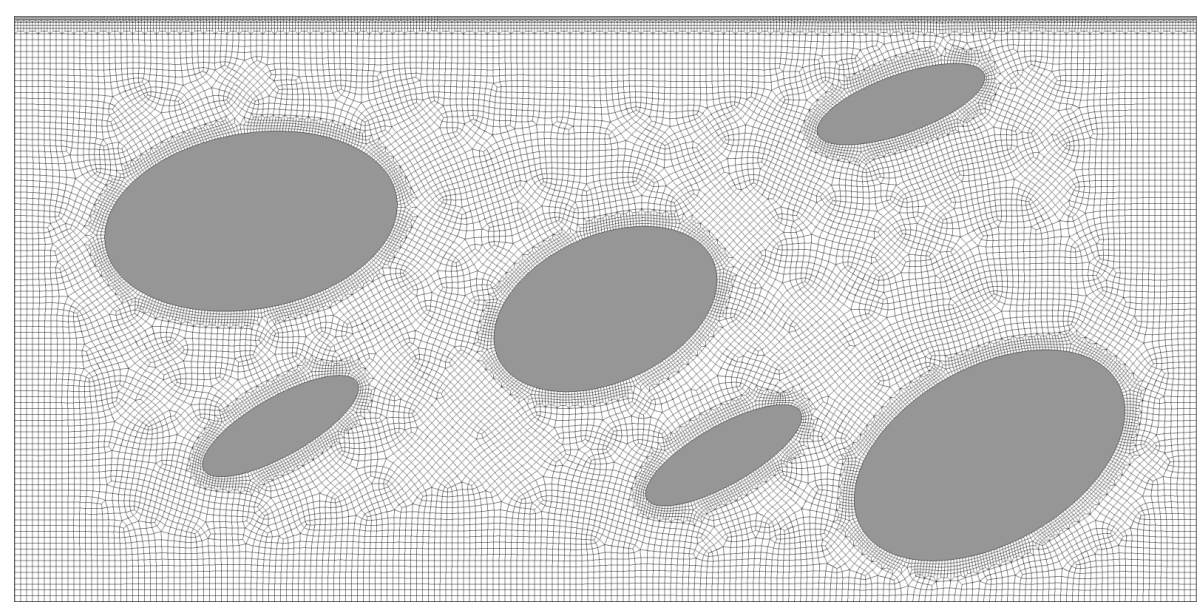

(c) Mesh detail in the case $\bar{K}_{2}=0$, for which the domain has impermeable obstructions.

Figure 6: Geometry and meshes for a general domain with embedded elliptical obstructions. The subsurface model is constituted of a rectangular domain of size $4 \mathrm{~m} \times 2 \mathrm{~m}$, in which are positioned a number of elliptical obstructions (in red) of different permeabilities. Details of the meshes in the cases $\bar{K}_{2}=\bar{K}_{1} / 10$ (less permeable obstructions) and $\bar{K}_{2}=0$ (impermeable obstructions) are also shown. 


\subsection{Dissolution of $\mathrm{CO}_{2}$ into brine aquifers and carbon sequestration}

In recent years, the idea of sequestering carbon dioxide for an indefinite time by dissolution into brine aquifers has been considered as a way to reestablish the carbon balance in the atmosphere [253, 141]. Because carbon dioxide is lighter than water, in a typical subsurface configuration, a layer of $\mathrm{CO}_{2}$ is trapped between cap rock (above, overburden) and the brine (below, underburden). Dissolution processes occur at the interface between $\mathrm{CO}_{2}$ and brine. As a result, $\mathrm{CO}_{2}$-rich, heavier brine is found near the top of the water table, with lighter brine lying below. As already commented when analyzing condition $(66)$ in Section 2.5 , this mechanical configuration is prone to the onset of buoyant instabilities, commonly referred as gravity-driven viscous fingering phenomena, which involve single-phase multi-component flow transport. The stability of gravity-driven instabilities, as already mentioned in Section 2.5 has received considerable attention [138, 134, 139, 140, 35, 137, 141, 142], and, more recently, numerical computations have been used to complement and refine theoretical findings. The numerical methods utilized in [138, 134, 139, 140, 35, 137, 141, 142], however, rely almost exclusively on Fourier methods or high-order finitedifference/finite-volume methods, which can maintain high accuracy only on Cartesian, or nearly-Cartesian grids. While these contributions have proved fundamental in improving the physical understanding of gravity-driven viscous fingering, the computational methods adopted somewhat lack the flexibility to attack the complex geometries of practical engineering subsurface problems.

The authors of [249, 250] proposed a high-order DG discretization for the simulation of gravity-driven viscous fingering in miscible displacement flows. The proposed methods enable high-fidelity, near-well (local) analysis and simulation in complex geometries and unstructured grids, without resorting to embedded/immersed boundary techniques. The numerical methods developed in [249, 250] were limited to single-phase flows, but their scope can easily be extended to multicomponent, multiphase flows with hysteresis and compressibility [253], which are the more general setting for $\mathrm{CO}_{2}$ storage and EOR.

Specifically, the authors of [249, 250] pursued an IMPES approach, in which an SIPG discretization of equation (35f), namely,

$$
\mathrm{B}[\boldsymbol{\kappa} ; \mathbf{g} ; \wp(P)](P, w)+\mathrm{B}[(\tilde{\phi} \mathbf{D}) ; \mathbf{0} ; \wp(P)](1 / \rho, w)=\mathrm{L}\left[\tilde{\boldsymbol{\kappa}} ; \tilde{\mathbf{g}} ; \sum_{i=1}^{N_{c}} \frac{q_{i}}{\rho_{i}} ; \wp(P)\right](w),
$$

is coupled with the following variational transport equation

$$
\begin{aligned}
\left(w, \partial_{t}\left(\tilde{\phi} C_{i}\right)\right)_{\Omega}+\mathrm{B}[\tilde{\mathbf{U}}]\left(C_{i}, w\right)_{U}^{a d v}+\mathrm{B}\left[(\tilde{\phi} \mathbf{D}) ; \mathbf{0} ; \wp\left(C_{i}\right)\right]\left(C_{i}, w\right)_{D G ; \xi=1 / 2}= & \mathrm{L}[\tilde{\mathbf{U}}](w)_{U}^{a d v} \\
& +\mathrm{L}\left[(\tilde{\phi} \mathbf{D}) ; \mathbf{0} ; \wp\left(C_{i}\right)\right](w)_{D G ; \xi=1 / 2},
\end{aligned}
$$

updated in time with a fourth-order explicit Runge-Kutta (RK4) time integrator. This approach is subject to a CFL condition, which can be more and more restrictive as the polynomial order is increased. It was observed in [249, 250] that if the pressure is solved at the very first stage of the RK4 integration procedure, and then frozen for the remaining RK4 stages, the computational cost is considerably reduced, with no apparent loss of accuracy. Another important point to be made is that if Legendre interpolation is used, the mass matrix associated with the DG formulation is diagonal, and no matrix inversion is required in the transport equation when an explicit integrator is used.

It is interesting to observe that this formulation does not satisfy the sum compatibility principle, and small spurious numerical artifacts/inconsistencies may be present on coarse grid computations. However, the calculation presented in [249, 250] were relatively fine, and do not show any of these issues.

In [249], optimal convergence rates were documented for pressure and concentrations, together with a detailed set of mesh refinement studies to assess the level of resolution necessary to correctly capture the physical mechanisms at the onset and later development of viscous fingers. We report here some of the results from [249, 250], to which the reader can refer for more details.

The flow conditions are similar to the one proposed in [141] for viscous fingering instabilities and corresponds to the conditions of a fluid in a porous medium at $45^{\circ} \mathrm{C}$ of temperature and 100 bar of pressure, as encountered in the Earth crust near $1000 \mathrm{~m}$ of depth. While the computations in [141] were limited to simple Cartesian geometries, the setup in [249, 250] is depicted in Figure 6(a), where a number of elliptical obstructions (in red) are embedded in a background domain $\Omega$ of size $4 \mathrm{~m} \times 2 \mathrm{~m}$ (in beige). The average permeability of the background domain is $\bar{K}_{1}=$ $10 \mathrm{D}=9.87 \times 10^{-12} \mathrm{~m}^{2}$, and we have considered three possible values for the average permeability of the obstructions: 


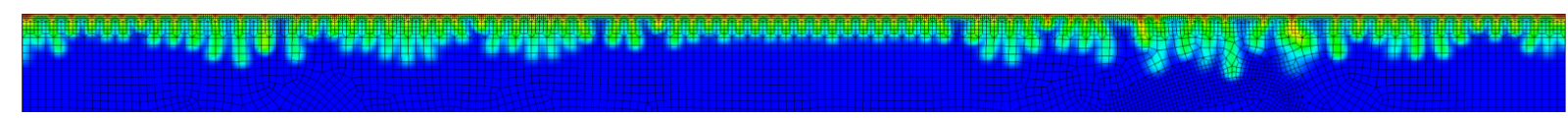

(a) $\bar{K}_{2}=\bar{K}_{1}$ (no obstructions).

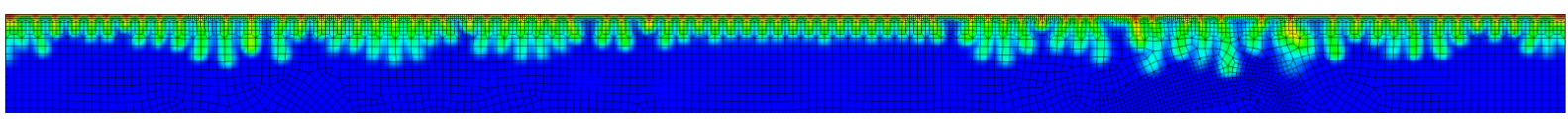

(b) $\bar{K}_{2}=\bar{K}_{1} / 10$ (low permeability obstructions).

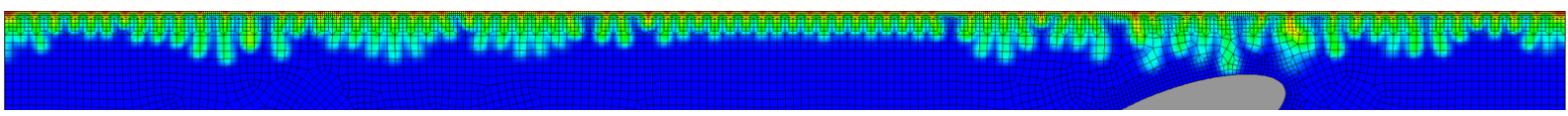

(c) $\bar{K}_{2}=0$ (impermeable obstructions).

Figure 7: Snapshots of the mass fraction of carbon dioxide at time $t=0.3 \times 10^{6} \mathrm{~s} \approx 3.5$ days, for the three distribution of permeability $\bar{K}_{2}$. Compare with Figure 8 where the time history of the carbon dioxide flux from the top boundary is computed.

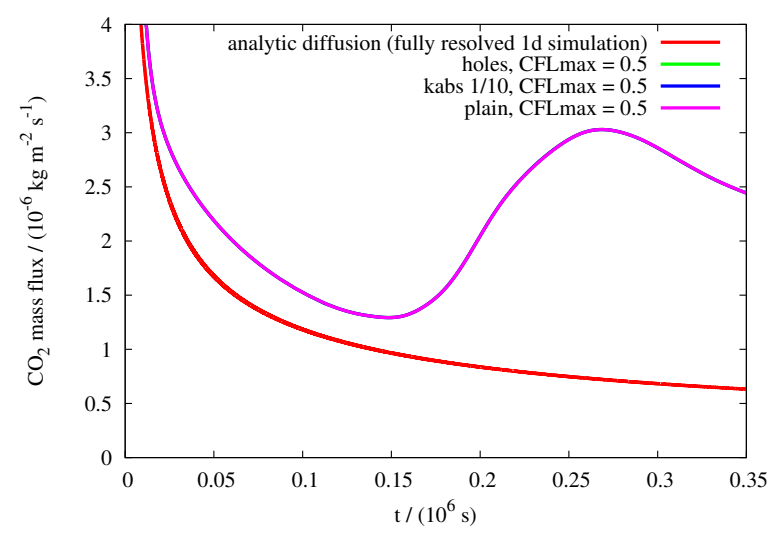

(a) Early transient.

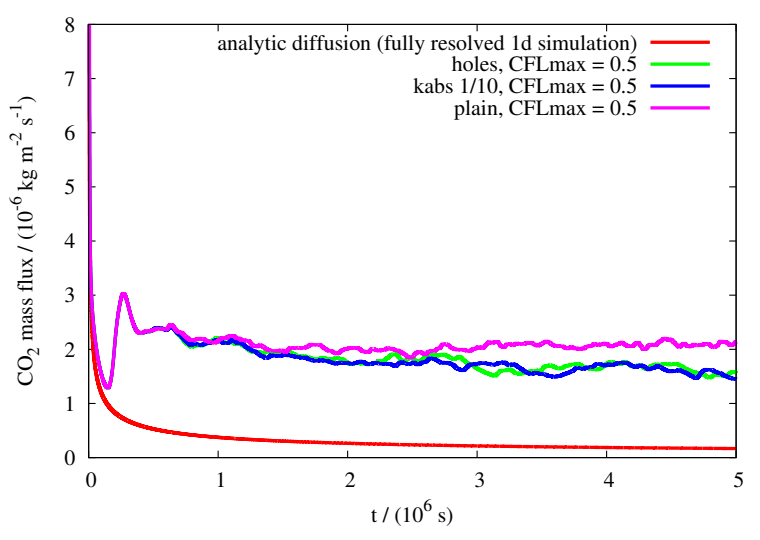

(b) Long term behavior.

Figure 8: Time history of the carbon dioxide flux from the top boundary, for the computations presented in Figures 7 and 9

$\bar{K}_{2}=\bar{K}_{1}$ (no obstructions, just an unstructured mesh), $\bar{K}_{2}=\bar{K}_{1} / 10$ (obstructions with moderate permeability), and $\bar{K}_{2}=0$ (fully impermeable obstructions). Impermeable obstructions $\left(\bar{K}_{2}=0\right)$ were modeled through impermeable boundary conditions, and the flow was not computed in these regions. The distribution of permeability was obtained by adding a $0.25 \%$ perturbation (in the $L^{2}$-norm) to the average permeability. Namely,

$$
\begin{aligned}
& K=(1+\alpha) \bar{K}, \\
& \alpha=0.025 \frac{2}{N} \sum_{n=1}^{N} \sin \left(2 \pi n x / L_{x}-\omega_{x}\right) \sin \left(2 \pi n y / L_{y}-\omega_{y}\right),
\end{aligned}
$$

with $N=8, L_{x}=4.0 \mathrm{~m}, L_{y}=2.0 \mathrm{~m}$, and $\omega_{x}$ and $\omega_{y}$ two uniformly distributed random numbers in the interval $[0,1]$. Note that in the limit as $N \rightarrow \infty$, the permeability $K$ would be perturbed with white noise of intensity 0.025 . The other parameters in the formulation are $\mu=0.5947 \times 10^{-3} \mathrm{~Pa}$ assumed constant, $\mathbf{D}=\lambda D_{0}$ (isotropic dispersion tensor) with $\lambda D_{0}=2 \times 10^{-9} \mathrm{~m}^{2} \mathrm{~s}^{-1}, \phi=0.3$, and $\mathbf{g}=[0.0,-9.81] \mathrm{m} \mathrm{s}^{-2}$.

The saturated carbon dioxide mass fraction is $\chi=0.049309$ and the density increase due to fully saturated carbon dioxide $\Delta \rho=10.45 \mathrm{~kg} \mathrm{~m}^{-3}$, which corresponds, substituting $\chi$ and $\rho_{2}=994.56 \mathrm{~kg} \mathrm{~m}^{-3}$ into (4), to $\rho_{1}=$ $1260.35 \mathrm{~kg} \mathrm{~m}^{-3}$. 


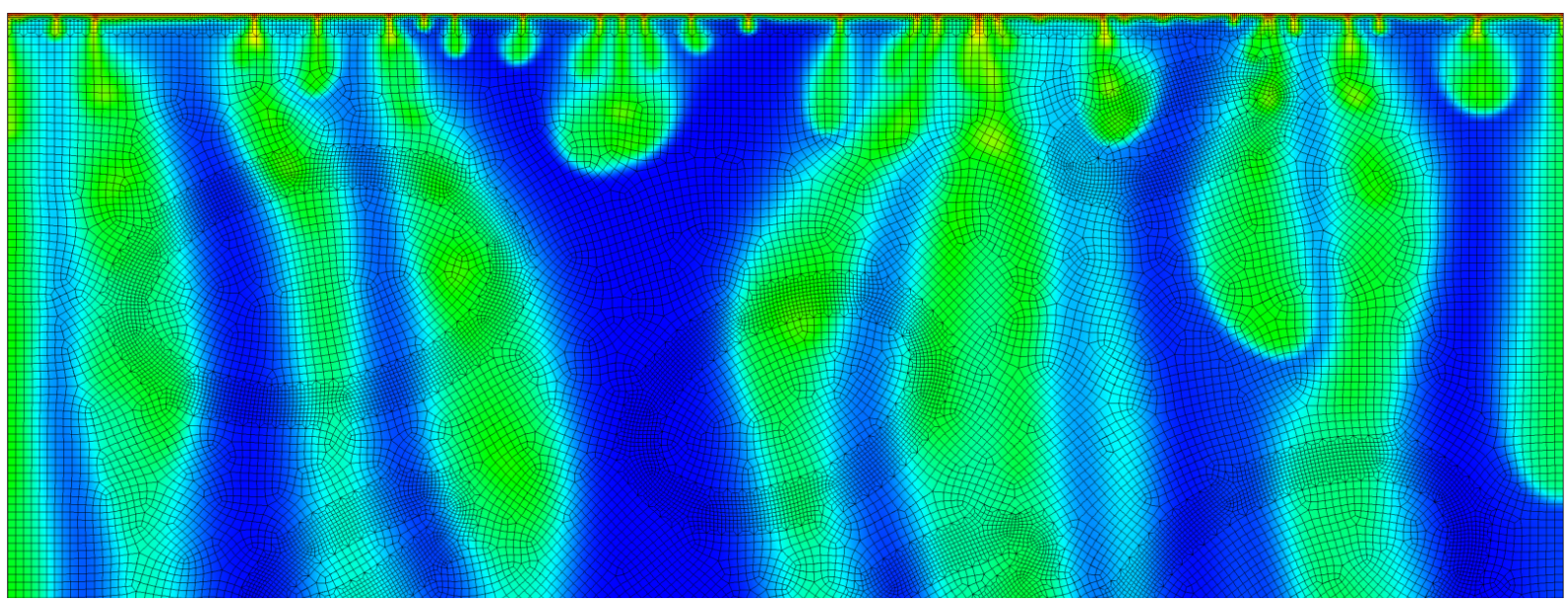

(a) $\bar{K}_{2}=\bar{K}_{1}$ (no obstructions).

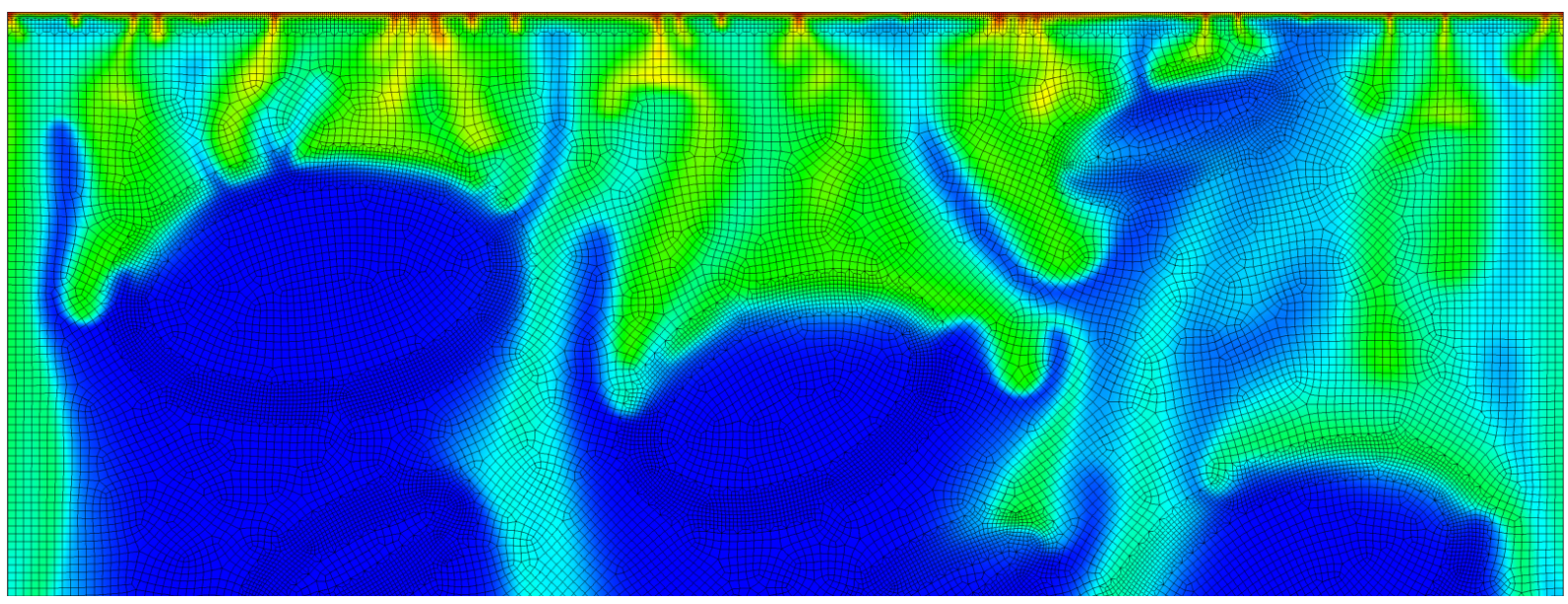

(b) $\bar{K}_{2}=\bar{K}_{1} / 10$ (low permeability obstructions).

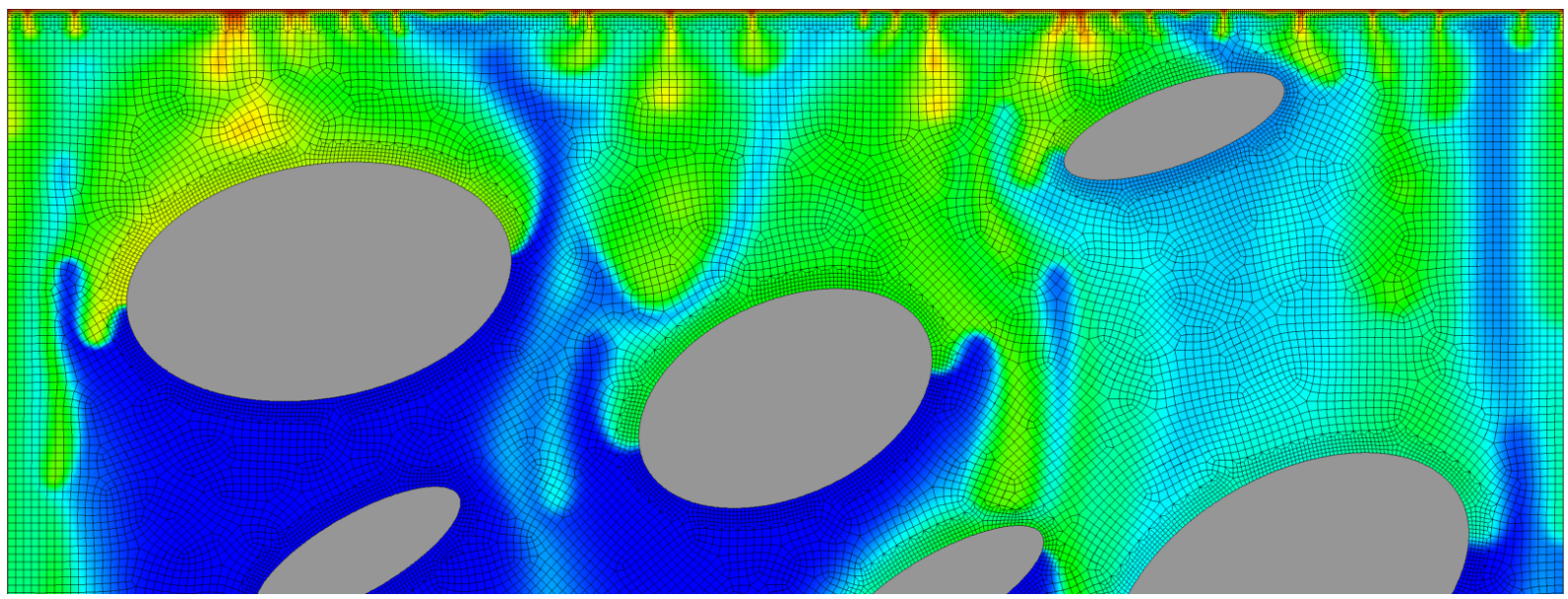

(c) $\bar{K}_{2}=0$ (impermeable obstructions).

Figure 9: Snapshots of the mass fraction of carbon dioxide at time $t=5.0 \times 10^{6} \mathrm{~s} \approx 58$ days, for the three distribution of permeability $\bar{K}_{2}$. Compare with Figure 8 where the time history of the carbon dioxide flux from the top boundary is computed. 
The boundary conditions for the pressure equation are given as no flow $(\mathbf{u} \cdot \mathbf{n}=0)$ on all sides of the rectangular domain except the bottom boundary, where the pressure is set to the reference value 0 (the pressure is in general defined up to a constant). In the case in which the inclusions are impermeable, they are modeled through no flow boundary conditions as well.

The boundary conditions for the transport equation are specified as homogeneous Neumann conditions on all but the top side, where the carbon dioxide mass fraction is in saturated conditions, that is $X_{1}=\chi=0.049309$ (Dirichlet conditions). The initial distribution of the carbon dioxide mass fraction is zero over the entire domain.

Remark 12. The size of the computational domain is chosen so that it contains a sufficiently large multiple of the fastest growing wavelength of the instability (in the horizontal direction.) Linear theory analysis [137, 141] predicts that the fastest growing mode corresponds to the wavelength

$$
\lambda_{c}=96.23 \frac{\phi \mu \tau \mathcal{D}}{K \Delta \rho g} \approx 0.03 \mathrm{~m},
$$

which yields that about 120 wavelengths of the most unstable (i.e., fastest growing, or critical) mode fit along the horizontal dimension.

All computations were performed using quadratic polynomials on fully unstructured meshes. Figure 7 shows an early stage of the computations: the flow has just reached the obstructions, and very little difference is observed in the flow patterns for the three different values of the permeability $\bar{K}_{2}$. Interestingly, also the values of the average flux from the top of the domain are virtually identical, as seen in Figure 8(a)

As time progresses and carbon dioxide dissolves and diffuses into the reservoir, the right hand side of (35f) becomes different from zero, and the specified perturbation in permeability causes minute flow velocities to form into the reservoir. These advection mechanisms are sufficient to start the formation of viscous fingers. At these very early stages, the dynamics are controlled by linear mechanisms, and only later on the system becomes fully nonlinear and chaotic behavior emerge.

Remark 13. It is worthwhile observing that the carbon dioxide flux in the very early stages $\left(t<10^{5} \mathrm{~s}\right)$ of the computation should closely match the one-dimensional analytical solution represented by the red curve in Figure 8(a), corresponding to a "thermal shock" pattern with extremely high gradients. This is an indication that the computational grid may still be slightly coarse near the top boundary, although it looks very refined to the eye. After the onset of instability $\left(t>2 \times 10^{5} \mathrm{~s}\right)$, however, the resolution is completely adequate to capture the fingering dynamics. See [249] for more details.

After some time, the obstructions deflect the flow in preferential channels, and the overall flux of carbon dioxide from the top boundary is reduced, although not very significantly (about 20\%.) It is also interesting to note that there is very little difference in the carbon dioxide average flux between the cases of $\bar{K}_{2}=\bar{K}_{1} / 10$ and $\bar{K}_{2}=0$ (impermeable obstructions). The initial system dynamics is in reality very simple: the flow is at rest and any change in carbon dioxide mass fraction occurs only due to diffusion processes. At the initial time, the pressure balances the gravity exactly, that is the right hand side of (35f) vanishes, and the system is in an (unstable) equilibrium configuration.

While the chosen shapes of the obstruction are somewhat arbitrary, their smooth contours allow for very interesting phenomena to occur, such as the oscillating shedding of viscous plumes from the boundary of the obstructions. This results in complex umbrella-like structures which develop from the competition between inertia and buoyancy forces, as seen in Figure 9. There, it is also easy to appreciate the smoothness of the solution. This is a quite interesting result, considering that the element size of the unstructured mesh changes quite rapidly near the regions where an abrupt variation in permeability occurs.

\subsection{First Contact Miscible Displacement in EOR processes}

Viscous fingering phenomena have received considerable attention in enhanced oil recovery (EOR) processes, because of their negative impact on the overall efficiency of the oil displacement [254, 255, 256, 257]. Somewhat surprisingly, other (only apparently) unrelated systems share a similar behavior. For example, the study of carbonate rock acidization in advanced EOR leads to a set of governing equations similar to the ones for miscible displacement 
flow. Also in this case, fingering, meandering flow structures called acidizing wormholes appear, and are governed by a dynamics analogous to the one of viscous fingers [258, 259, 260, 261, 262, 263, 264, 265, 266].

In this paper, we have restricted our attention to two component single phase flow. In oil recovery, this model often refers to first contact miscible displacement, in which injection fluids mix directly with reservoir oils in all proportions and their mixtures remain single phase. LPG (liquid petroleum gas) such as ethane propane and butane are the common solvents for first contact miscible flooding. [267].

\subsubsection{Early simulations with finite difference schemes}

Early computational studies of miscible displacement problems [19, 132, 268, 269, 270] were often performed by coupling finite differences schemes for the flow equations with particle tracking methods for the transport equations. Here random-walk particle tracking schemes that treat dispersion as a random process were used to solve transport [19, 271] by casting a convection-dispersion equation into the form of a stochastic Fokker-Planck equation [272]. The experimental observations and numerical simulations of stable and unstable displacement experiments in millstone and limestone cores were shown in [19]. Advances in computing power extended the numerical simulations to three dimensional flow in [268, 269, 10, 270, 273, 274]. Mixed finite-difference was coupled with particle tracking [13], finite difference schemes with flux corrected transport schemes [72], and spectral methods based on vorticity formulations [5, 12] were also used.

\subsubsection{Mixed finite elements for flow}

Mixed methods were introduced to improve the quality of numerical solutions, by means of accurate flow velocities in the $H($ div $)$ space, local conservation, and continuity of the fluxes across element edges/faces. In the earliest examples [275, 276], mixed methods for flow were combined with the SIPG method for transport. Later, the NIPG method was used in [277] and applications to multiphase flow were also considered in [278, 279]. Mixed methods were also combined with the modified method of characteristics in [233, 234, 231, 280]. These approaches were computationally efficient and allowed for very large time steps, but also suffered from some problematic issues on the imposition of boundary conditions, and backtracking flow near wells. In [233, 234, 280], numerical simulations of unstable viscous fingering were performed, with studies of growth rates and budgets of the number of active fingers, under the effect of gravity. Figure 10 illustrates results for a quarter five spot simulation with a discontinuous permeability field by employing mixed finite elements for flow and SIPG for transport. Figure 11 shows computations of fingering patterns produced by means of a random permeability field, employing mixed finite elements for flow and the modified method of characteristics for transport. Figure 12 presents the recovery curve for different viscosity ratios at the production well. Figure 13 shows results for an unstable displacement problem where the permeability is set to match the ARCO Core 1 rock.

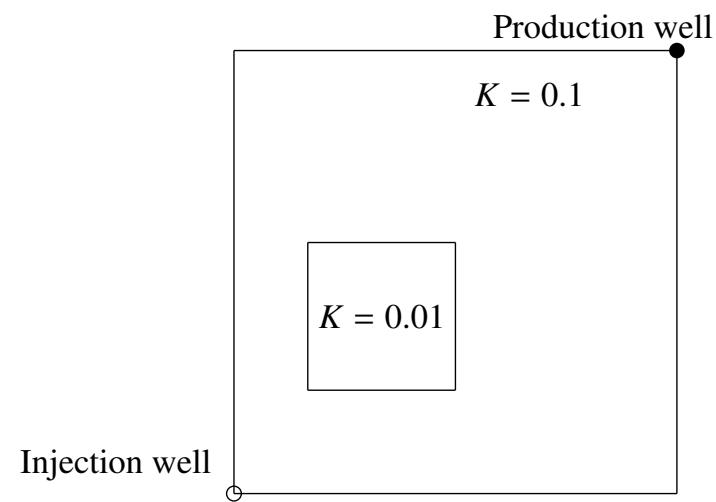

(a) Domain

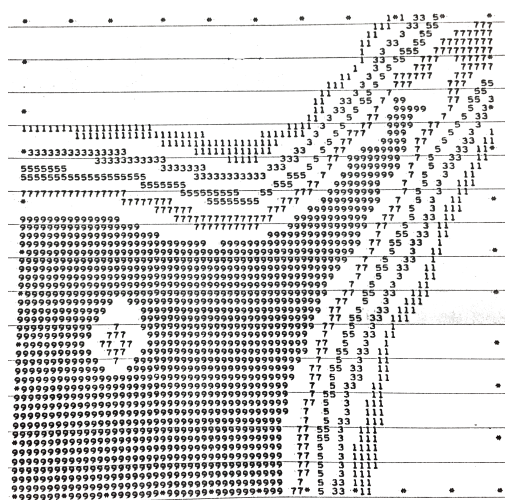

(b) Contour values

Figure 10: The quarter five-spot problem with discontinuous permeability field with miscible displacement flow. Solutions are computed by coupling mixed finite elements (flow) and the SIPG method (transport). Courtesy of Mary F. Wheeler. 


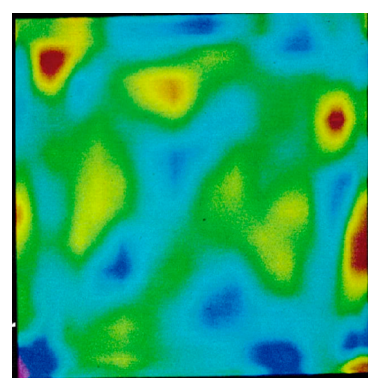

(a) Permeability

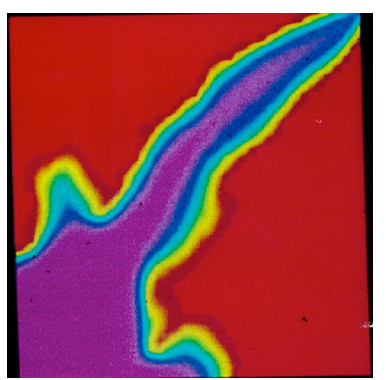

(b) Concentration values

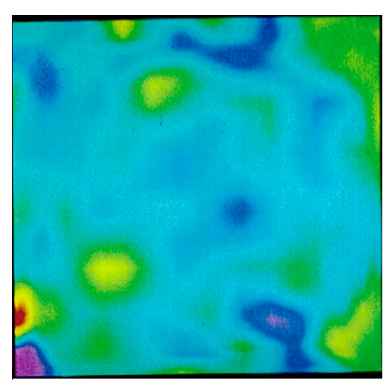

(c) Permeability

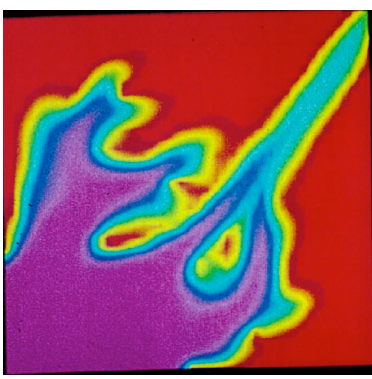

(d) Concentration values

Figure 11: The quarter five-spot problem with miscible displacement flow. Solutions are computed with mixed finite elements (flow) and the modified method of characteristics (transport). Concentration values (b) and (d) at time 1400 days are illustrated with two different random permeability fields (a) and (c), respectively. The viscosity ratio is 41 . Courtesy of Mary F. Wheeler.

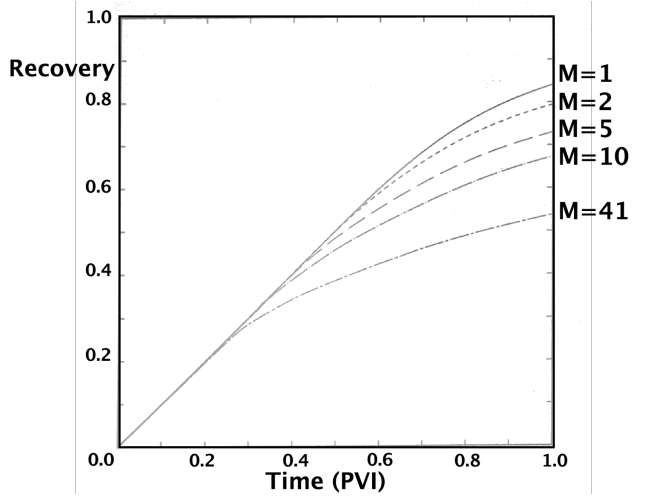

Figure 12: Example of miscible displacements with different viscosity ratios $(M=1,2,5,10$ and 41$)$. Recovery curves at the production well versus time (PVI). Courtesy of Mary F. Wheeler.
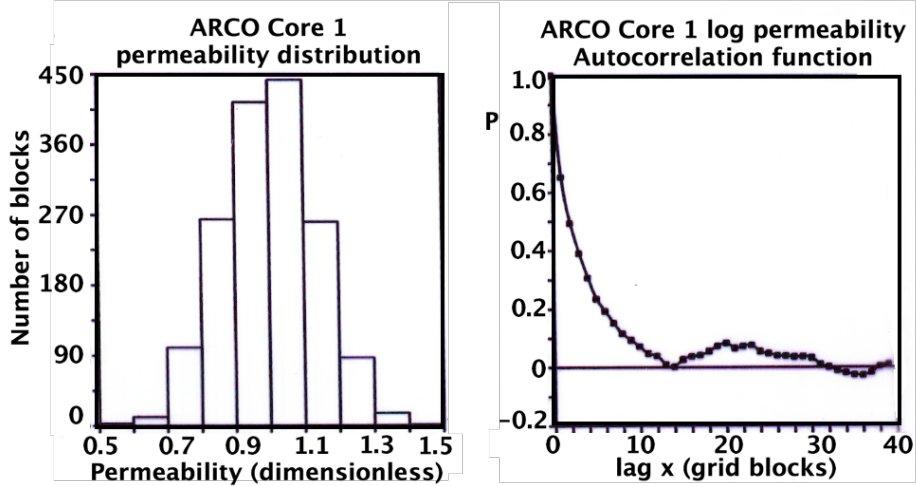

(a) ARCO Core 1 permeability

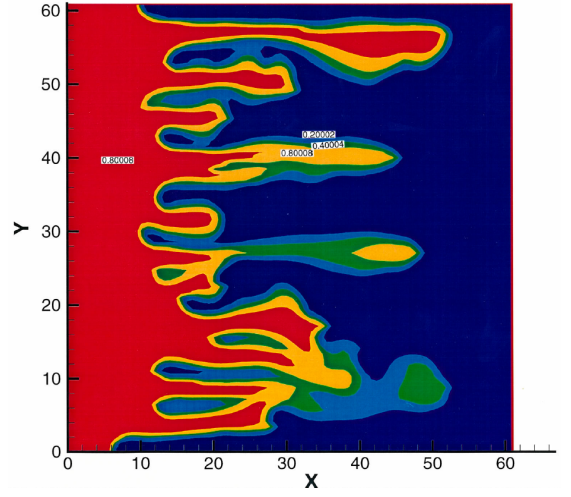

(b) Unstable miscible displacement

Figure 13: Example of unstable miscible displacement flow. (a) Permeability data for ARCO Core 1 rock. (b) Concentration values for unstable miscible displacement with ARCO permeability and viscosity ratio 75 at $\mathrm{t}=0.45 \mathrm{PV}$. Courtesy of Mary F. Wheeler

\subsubsection{Fully coupled discontinuous Galerkin methods}

In 2013, the authors of [157, 158] proposed a high-order DG method to simulate viscous fingering problems for miscible compressible and incompressible displacement flow. This approach features a fully implicit coupling of the 
flow and species transport equations, and the UC-DG formulation described in Section 4.1.8 and 4.2.4, for both the species transport and flow equations. This work was one of the first to highlight the potential of higher-order DG discretizations in the context of viscous fingering simulations, and was the first to propose the idea of the discrete sum compatibility principle when solving coupled flow/transport problems. More recent work [281, 282] featured higher-order DG methods for miscible displacement viscous fingering problems in heterogeneous porous media.

The authors of [158, 157] coupled (137)-(138) using the upwind-biased compact DG method presented in Section 4.1.6, and solved the nonlinear system of equations with a monolithic Newton solver (i.e., with the fully implicit coupling approach of Section 3.1.2. Other options are also possible, such as, for example, IMPES or sequentially coupled implementations. As already observed in Section 4.1.6 the discretization utilized in [158, 157] satisfies the DSC principle.

In what follows, we present results from computations performed in the case of a two-species system with no gravity, that is $\tilde{\mathbf{g}}=\mathbf{0}$. The dependency of the viscosity on the solute mass fraction is given by

$$
\mu=e^{R(1-c)},
$$

where $R$ can be interpreted as the logarithm of the mobility contrast [4, 5, 6, 8, 10, 11, 14, 15, 16, 283, 17, 18, 19]. The numerical studies in [158, 157] showed that mass fractions (concentrations) and pressures converge with order $k+1$ if polynomial interpolation of order $k$ is used for both these discrete fields. It was also shown that the computed growth rates in the early stages of viscous fingering instabilities are correctly captured if interpolation of order $k \geq 1$ is used. In [158, 157] it was also shown that calculations performed with $k=0$ corresponded to an upwind first-order finite volume method and did not correctly capture the expected growth rates from the linearized theory.

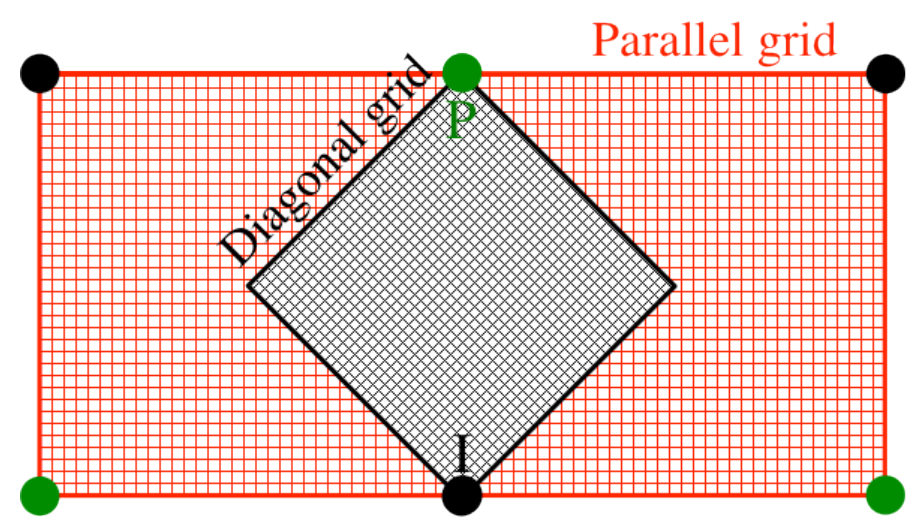

(a) Problem setup.

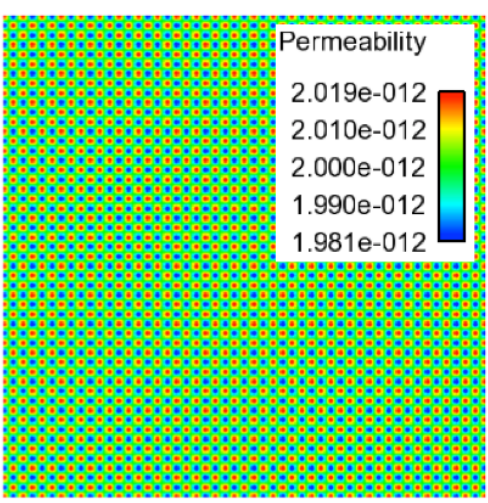

(b) Permeability perturbation.

Figure 14: Setup of a mesh sensitivity test. The same flow solution is computed on two different domains, with meshes that differ in orientation. In the case of the "parallel" grid in red, the flow predominantly moves between an injection well (denoted by a the black "I") to a production well (denoted by a green "P") along a direction parallel to some of the mesh lines. In the case of the "diagonal" grid in black, the flow predominantly moves along a direction diagonal to the mesh lines. The permeability distribution is shown in Figure 14(b) and is given by [144a], and it does not depend on the grid orientation.

Mesh orientation studies. In practical reservoir engineering computations, the complex geometry of the subsurface may require fully unstructured meshes. It becomes then very important for a numerical method to be insensitive to grid orientation. This property is not necessarily related to the robustness of the method, rather, to its level of fidelity. Consider the setup depicted in Figure 14(a), in which the same five-spot injection problem is computed on two different grids, denoted as "diagonal" and "parallel," depending on whether the dominant flow direction is diagonal or parallel to the grid lines. We "seed" the initial mass fraction distribution with an exact solution of the incompressible miscible displacement equations proposed in [7], and given by 


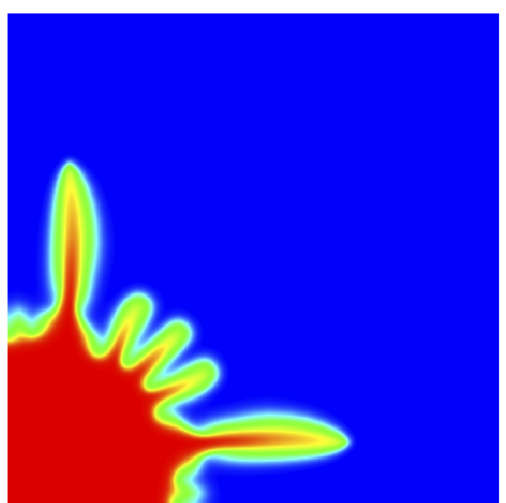

(a) $k=0, h=2.5 \times 10^{-3}$, "parallel" grid.

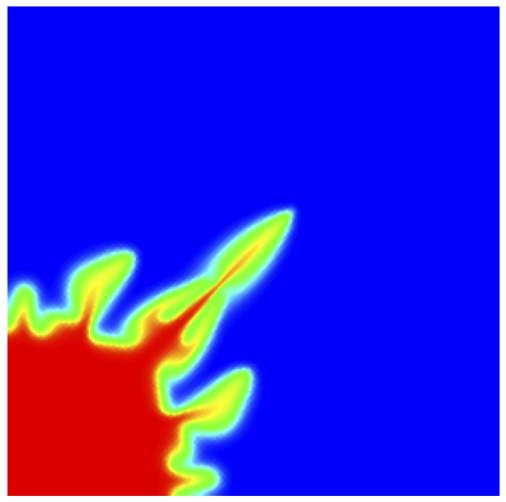

(d) $k=0, h=2.5 \times 10^{-3}$, "diagonal" grid.

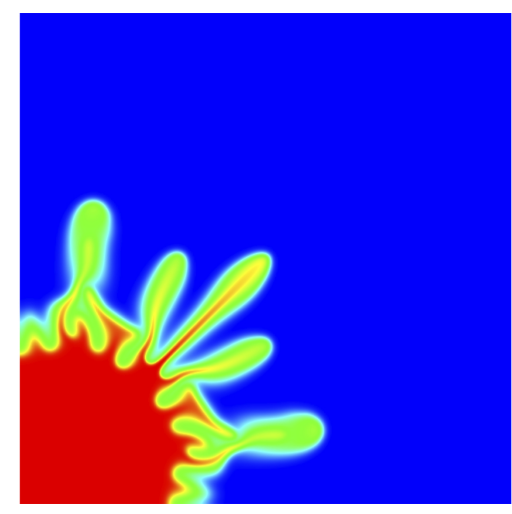

(b) $k=1, h=3.33 \times 10^{-3}$, "parallel" grid.

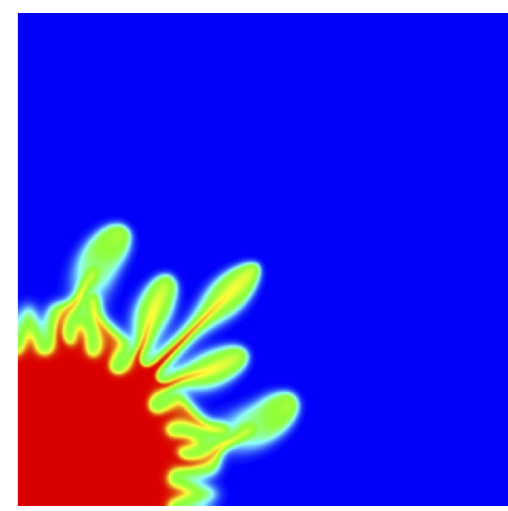

(e) $k=1, h=3.33 \times 10^{-3}$, "diagonal" grid.

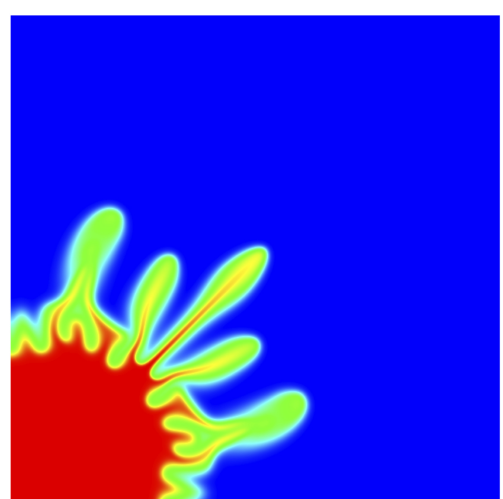

(c) $k=4, h=8.33 \times 10^{-3}$, "parallel" grid.

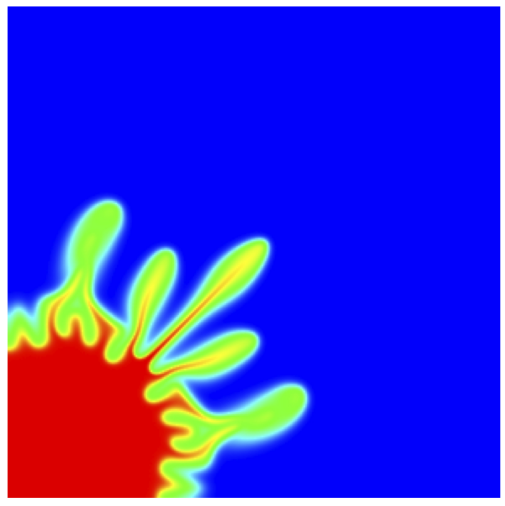

(f) $k=4, h=8.33 \times 10^{-3}$, “diagonal” grid.

Figure 15: Mesh orientation sensitivity test. All solutions are sampled at time $T=80,740 \mathrm{~s}$. The injection well is on the lower left corner and the production well is on the top right corner. Only the region of the domain corresponding to the black subdomain in Figure 14(a) is shown. Note that the method of order $k=0$ (corresponding to an upwind first-order finite volume discretization) shows extreme sensitivity to mesh orientation, even for fine-grained grids. In contrast, the results of the UC-DG method with $k=4$ are mesh-independent. The results of the UC-DG method with $k=1$ show very moderate mesh sensitivity.

$$
\begin{aligned}
u_{r}^{0}(r) & =\frac{1}{r}, \\
u_{\theta}^{0} & =0, \\
c^{0}(r, t) & =\frac{\int_{0}^{\eta} s^{P e-1} e^{s^{2} / 2} d s}{\int_{0}^{\infty} s^{P e-1} e^{s^{2} / 2} d s},
\end{aligned}
$$

where $u_{r}$ and $u_{\theta}$ are the radial and tangential components of $\mathbf{u}$, respectively, and the radius $r$ is measured with respect to the injection point "I" in Figure 14(a) In addition, $\mathbf{D}=\left(\lambda D_{0}\right) \mathbf{I}$ with $\lambda D_{0}=2.55 \times 10^{-9}, \eta=r(P e / 2 t)^{1 / 2}$, and $P e=Q /\left(\lambda D_{0}\right)=2 \pi u_{r} r /\left(\lambda D_{0}\right)=2 \pi /\left(\lambda D_{0}\right)=400$ is the global Péclet number, with $Q=1.0186 \times 10^{-6}$. The logarithmic mobility ratio is chosen as $R=5>0$. The permeability tensor is assumed isotropic, that is $\mathbf{K}=K \mathbf{I}$, and it is constituted of a constant field with a small (1\%) perturbation superposed, that is

$$
\begin{aligned}
K & =(1+\alpha) \bar{K}, \\
\bar{K} & =2.0 \times 10^{-12}, \\
\alpha & =10^{-2} \cos (50 \pi x / L) \cos (50 \pi y / L),
\end{aligned}
$$

where $L$ is the length of the side of the black square domain depicted in Figure 14. Figure 14(b) shows the distribution of the permeability parameter $\kappa$ in the diagonal grid. 


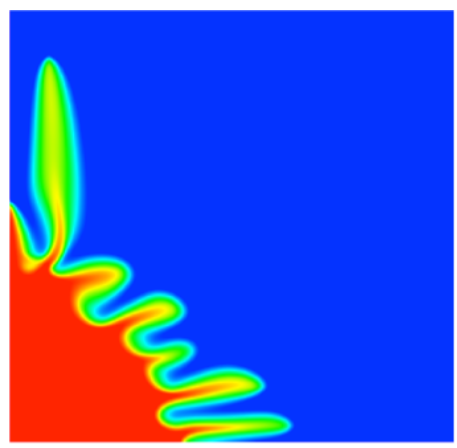

(a) $k=0, h=0.0025, K_{22} / K_{11}=0.5$.

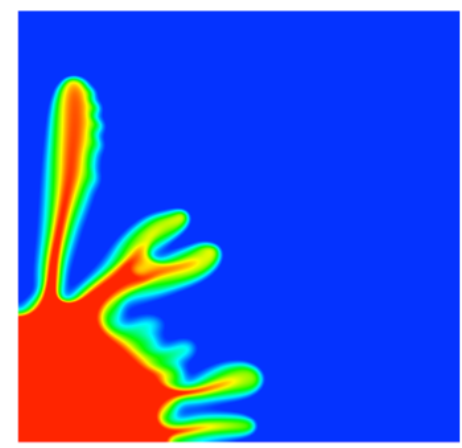

(b) $k=1, h=0.0025, K_{22} / K_{11}=0.5$.

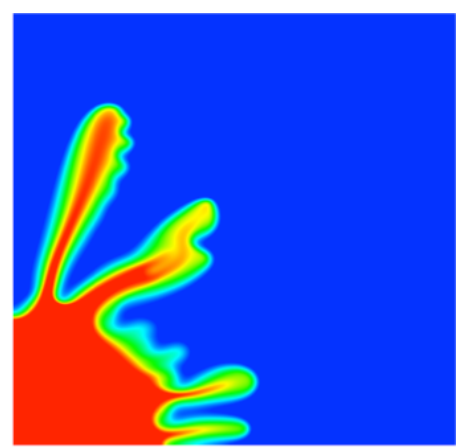

(c) $k=2, h=0.004, K_{22} / K_{11}=0.5$.

Figure 16: Effect of anisotropy in permeability. Results of simulations on uniform quadrilateral grids at $T=1.1464 \times 10^{5}$, for a compressibility coefficient $C_{\phi}=10^{-7} \mathrm{~Pa}^{-1}$ and a $1 \%$ magnitude of the permeability perturbation. Note that the solutions for $k=1$ and $k=2$ are similar, and very different from the case $k=0$ (upwind $\mathrm{FV}$ ), which is affected by mesh sensitivity issues.

This pattern is extended by continuity over the entire domain in red. Note in particular that due to the symmetries in the configuration of the red (parallel) grid problem, the boundary of the diagonal grid is aligned with streamlines in the flow. Figure 15 shows the results of computations performed with an upwind first-order finite volume discretization, which, as shown in [157], is identical to the UC-DG with piecewise constant interpolation, $k=0$. The numerical solution, in this case, is highly sensitive to mesh orientation, since viscous fingers propagate predominantly in directions parallel to element edges.

Instead, in the case of the UC-DG method with $k \geq 1$ the sensitivity of the solution to the mesh orientation is minimal. In flow instability phenomena such as viscous fingering, small numerical differences (e.g., the interpolation error on the initial solution) grow at a very fast pace, so that one cannot expect to deterministically track the solution for long times. It is then quite surprising that, in the case $k \geq 1$, results of computations with different mesh orientations are very close for considerably long times, at which nonlinear dynamics dominates.

Effect of anisotropic permeability. Figure 16 shows results when anisotropy is introduced in the permeability tensor, in the form

$$
\mathbf{K}=(1+\alpha)\left[\begin{array}{cc}
K_{11} & 0 \\
0 & K_{22}
\end{array}\right],
$$

with $\sqrt{K_{11} K_{22}}=10^{-12} \mathrm{~m}^{2}$, and $K_{22}=0.5 K_{11}$. Computations are performed on the same domain and with identical initial conditions used in the previous section, and on grids composed of square elements. The boundary conditions are slightly different, in that no-flow Neumann conditions are imposed on all sides of the square domain.

Also in the case of this set of tests, it is clearly visible the strong mesh dependence of the numerical results for polynomial order $k=0$ : fingers protrude only in the vertical or horizontal directions. Computations performed with $k=1$ and $k=2$ (we only show results for $k=2$ for the sake of brevity) do not suffer from mesh orientation artifacts, and are in fair agreement with each other. In this case, fingers are allowed to grow also in directions which form an arbitrary angle with the grid lines. The higher permeability in the horizontal direction promotes lateral growth of vertical fingers and allows wider pathways for the injected fluid. As a result, we observed a few thicker and longer vertical fingers. In contrast, horizontal fingers are more constrained in lateral expansion due to lower vertical permeability, and appear relatively shorter due to the lack of solvent supplies.

General domains with unstructured grids. The case of a more complex computational domain, with a setting very similar to the five-spot problem, is presented in Figure 17(a) and 17(d) The boundary and initial conditions are as before, but with the difference now that an impermeable obstruction of elliptic shape is placed in the middle of the domain. Results are shown for approximations of order $k=0$ and $k=2$, for the incompressible case, and the compressible case with a compressibility coefficient $C_{\phi}=10^{-8} \mathrm{~Pa}^{-1}$. The solutions for $k=0$ are computed on a 


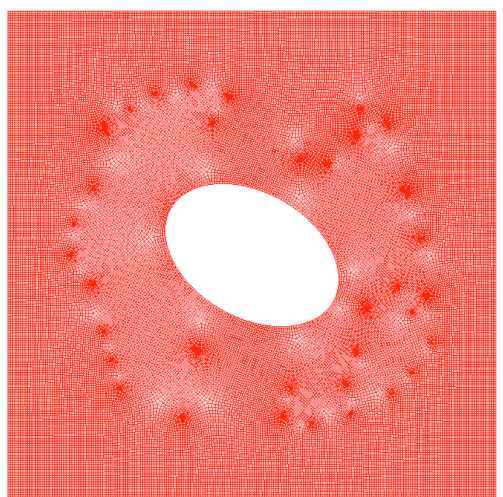

(a) Unstructured grid

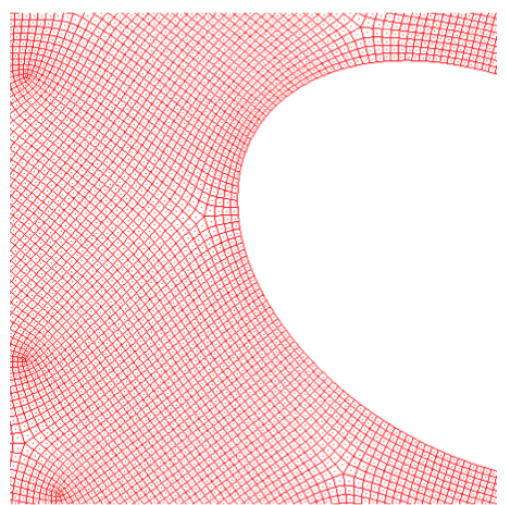

(d) Zoomed detail of the grid

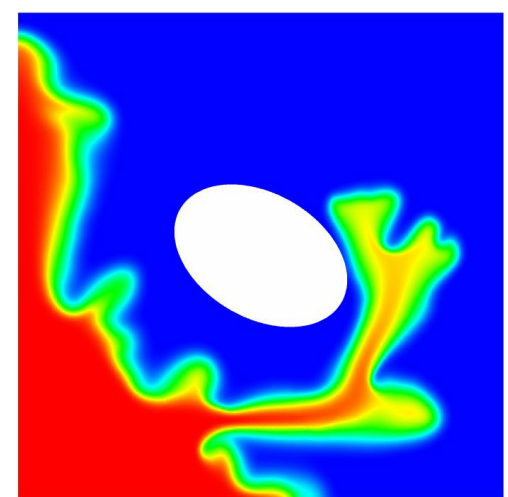

(b) $k=0, T=1.436 \times 10^{4} \mathrm{~s}, C_{\phi}=0 \mathrm{~Pa}^{-1}$

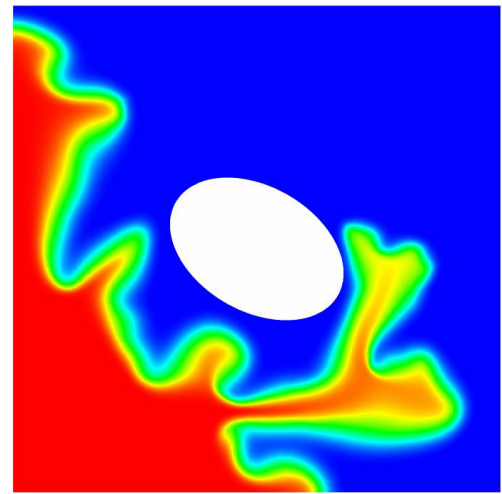

(e) $k=0, T=2.244 \times 10^{4} \mathrm{~s}, C_{\phi}=10^{-8} \mathrm{~Pa}^{-1}$

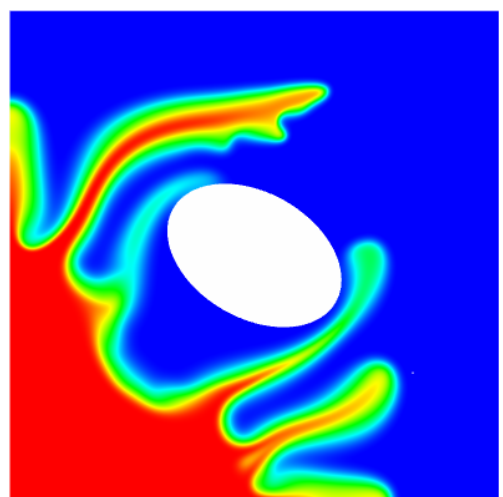

(c) $k=2, T=1.436 \times 10^{4} \mathrm{~s}, C_{\phi}=0 \mathrm{~Pa}^{-1}$

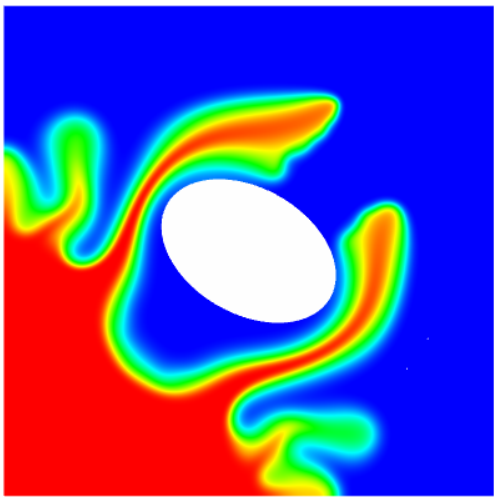

(f) $k=2, T=2.244 \times 10^{4} \mathrm{~s}, C_{\phi}=10^{-8} \mathrm{~Pa}^{-1}$

Figure 17: Computations with fully unstructured grids on a domain with an elliptic obstruction. Figure 17(a) shows one of the computational grids used, Figure 17(d) shows a detail of the unstructured grid. Simulations with $k=0$ and $k=2$ were performed using 150,912 and 37,728 elements, respectively. Both incompressible and slightly incompressible media are considered.

grid of 150,912 elements, while the solution for $k=2$ is computed on a grid of only 37,728 elements, so that the overall resolution is similar. The results for $k=0$ clearly show strong sensitivity to the mesh orientation, as the fingers develop primarily in the vertical and horizontal directions. For fully unstructured grids, the equivalence between DG with $k=0$ and an upwind finite volume discretization is not strict, but it is still easy to see that the unstructured grid in Figure 17(a) and 17(d) is composed of patches of nearly Cartesian grids. The solutions for $k=2$ do not show sensitivity to mesh orientation. This is a very important result, since it demonstrates that the conclusions outlined in the previous section hold also in the case of general meshes, independent of compressibility effects. Note also that the development of viscous fingers is retarded as the compressibility of the medium increases.

\subsection{Ground water contamination and bioremediation}

Contamination of aquifers from sources such as improperly disposed toxic waste, leaking storage tanks, seepage from pollute streams and ponds is a major public concern. The decomposition of contaminants by microorganisms, or microbial biodegradation, is an important natural process that can be accelerated to protect a potable water supply. This system is physically and chemically complex, involving flow, transport, and the interaction of various hydrocarbons and microbes with water movement within the aquifer [284].

A related example of waste remediation system is the Hanford Site in Washington State, which occupies approximately 560 square miles of semiarid terrain and was selected for producing plutonium in support of the United States' World War II efforts [285, 286]. Chemical processes employed to recover and purify plutonium produced waste containing actinide compounds and aqueous and organic liquid industrial wastes. When plutonium production ceased, 

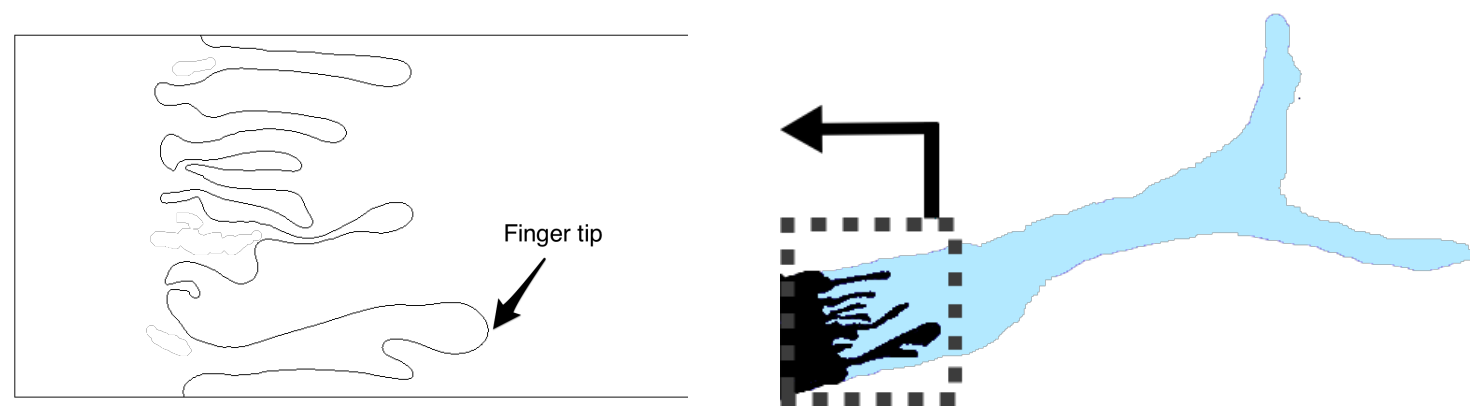

Figure 18: Fracture propagation with proppant filling. In the early stages of the injection, the interface between proppant-rich fluid and background fluid is prone to viscous fingering instabilities. Proppants then migrate rapidly to the fracture tip, helped by the fingers' flow pattern.

the primary mission and concern shifted to environmental restoration of the Hanford Site. In [287], DG methods for both flow and transport and mesh adaptation were combined to simulate of reactive transport flows, with applications to nuclear waste storage in a heterogeneous porous medium as a subsurface repository, and with a particular focus on the French ANDRA complex. Examples of simulations of bioremediation can be found in [288, 289, 290].

The transport equation introduced in (1) needs to be modified in order to be extended to applications just mentioned:

$$
\frac{\partial}{\partial t}\left(\phi \rho c_{i}\right)+\nabla \cdot\left(\rho \mathbf{u} c_{i}-\phi \rho \mathbf{D}(\mathbf{u}) \nabla c_{i}\right)+\lambda_{i} c_{i}=\phi_{i} r_{i}\left(c_{i}\right)+q_{i}, \text { in } \Omega \times(0, \mathbb{T}],
$$

where the nonlinear biogeochemical reaction operators $\lambda_{i} c_{i}$ and $r_{i}\left(c_{i}\right)$ have been introduced. For many of the applications just described, the flow is advection dominated and the time scale of advection and reaction are different, since reaction occurs much faster than advection. An operator splitting approach was applied in [284] to avoid numerical stiffness in the variational formulations: First, mixed finite elements were employed to compute the flow and a modified method of characteristic was used for treating advection-dispersion; then, applying a time-splitting algorithm, the reaction terms were incorporated in the solution strategy.

In [290], a procedure based on combining the modified method of characteristics with a Galerkin finite element method is analyzed for approximating reactive transport in groundwater. In particular, contaminant transport is modeled with nonlinear, non equilibrium adsorption reactions equations.

\subsection{Proppant-enhanced hydraulic fracture propagation}

In the general context of standard EOR processes viscous fingering is regarded as having a negative impact on oil production, and, therefore, something to be prevented (see also the discussion in Section 5.2). However, more recently, viscous fingering is regarded as beneficial for advanced EOR techniques based on fracture propagation in porous media [291, 292, 293]. Fracture-based EOR is a process in which, through the application of cyclic, very large pressures, fractures are propagated in the subsurface. This process is also known as hydraulic fracturing, and in order to keep the fractures open as they propagate, small grains of solid material, known as proppant are injected with the fracturing fluid. Proppants can be made of sand, treated sand or man-made ceramic materials, etc. With the goal of bringing proppants to the tip of the fracture in the smallest amount of time, viscous fingers can be helpful, since they create preferential mobility channels, as shown in Figure 18 Estimating the finger tip velocity becomes very important in this case, and this spurred a renewed interest in the theoretical and computational studies on the subject, already discussed in Section 2.6. According to experimental [294] and numerical studies [174], the speed of the finger tip may increases rapidly with the viscosity ratio, in the case of relatively viscosity ratios, and plateaus to an asymptotic limit for very large viscosity ratios.

In [174], EG approximations (see Sections 4.1.9 and 4.2.5) were employed for numerical simulations of viscous fingering in two and three dimensional Hele-Shaw cells, as a model for the proppants flowing inside hydraulic fractures. The sequential coupling approach described in Section 3.1.3 was used for the system of the flow and transport equations. We recall that the sequential coupling strategy is very effective in computations with heterogeneous permeabilities and velocity dependent dispersions. For each time step, first we seek the solution to the variational problem 
Find $P \in V_{h, k_{p}}^{\mathrm{EG}}\left(\mathcal{T}_{h}\right)$ such that

$$
\mathrm{B}[\tilde{\boldsymbol{\kappa}} ; \mathbf{0} ; \wp(P)](P, w)_{0, \xi}=\mathrm{L}[\tilde{\boldsymbol{\kappa}} ; \mathbf{0} ; \psi ; \wp(P)](w)_{0, \xi}, \quad \forall w \in V_{h, k_{p}}^{\mathrm{EG}}\left(\mathcal{T}_{h}\right),
$$

then, using the definition of the DG numerical fluxes [109, it is possible to obtain a conservative velocity flux variable $\mathbf{U}$. Once $\mathbf{U}$ is computed, one can proeed with the solution of

Find $C \in V_{h, k_{c}}^{\mathrm{EG}}\left(\mathcal{T}_{h}\right)$ such that

$$
\left(v, \partial_{t}(\tilde{\phi} C)\right)_{\Omega}+\mathrm{B}[\tilde{\mathbf{U}}](C, v)_{U}^{a d v}+\mathrm{B}[(\tilde{\phi} \mathbf{D}) ; \mathbf{0} ; \wp(C)](C, v)_{\mathrm{DG} ; \xi=1 / 2}=\mathcal{L}(v), \quad \forall v \in V_{h, k_{c}}^{\mathrm{EG}}\left(\mathcal{T}_{h}\right),
$$

where

$$
\begin{aligned}
\mathcal{L}(v):=\mathrm{L}[\tilde{\mathbf{U}}] & (v)_{U}^{a d v}+\mathrm{L}[(\tilde{\phi} \mathbf{D}) ; \mathbf{0} ; \wp(C)](v)_{\mathrm{DG} ; \xi=1 / 2} \\
& -\left(\mu_{\mathrm{Stab}_{\mid T}} \nabla C, \nabla v\right)_{\mathcal{T}_{h}}+\left\langle\left\{\left\{\mu_{\mathrm{Stab}_{\mid T}} \nabla C\right\}, \llbracket \llbracket v \rrbracket\right\rangle_{\Gamma_{e} \backslash \Gamma}+\varepsilon(k) h_{e}^{-1}\left\langle\left\{\left\{\mu_{\mathrm{Stab}_{\mid T}}\right\} \llbracket[C \rrbracket, \llbracket v \rrbracket\rangle_{\Gamma_{e} \backslash \Gamma} .\right.\right.\right.
\end{aligned}
$$

The last three terms in (148b) represent an entropy residual numerical stabilization operator [251], to prevent oscillations near sharp fronts. Here $\mu_{\mathrm{Stab} \mid T}$ denotes the piecewise-constant field of the entropy residual-based viscosity, which can be implemented implicitly or explicitly. More details are found in [295, 251, 174, 296]. We note also that the EG method employs DG variational formulations, but with fewer degrees of freedom. To satisfy a DSC principle, the IIPG $(\theta=0)$ method was employed for flow.

Numerical examples are illustrated in Figure 19 and Figure 20. Concentration contours for two- and threedimensional miscible displacement flow through a porous medium of random permeability are shown in Figure 19 A dynamic adaptive mesh refinement technique is also implemented using the entropy residuals for large-scale, efficient computations of three-dimensional flows.

Two- and three-dimensional viscous fingering in Hele-Shaw cells for different viscosity ratios are illustrated in Figure 20. The fluid viscosity is given by quarter-mixing rule [143]:

$$
\mu(c)=\frac{1}{c \mu_{s}^{-0.25}+(1-c) \mu_{o}^{-0.25}}, \quad \text { with } \quad \mu_{o} \geq \mu_{s},
$$

where $\mu_{s}$ is the solvent and $\mu_{0}$ is the viscosity of the residing fluid. Here $\mu_{s}$ is given as water and various $\mu_{0}$ are tested.

\section{Acknowledgments}

G. Scovazzi would like to acknowledge the support of: ExxonMobil Upstream Research Company (Houston, TX), The Department of Energy's Office of Science, Advanced Scientific Computing Program (through the Early Career Research Program Grant SC0012169 and the SciDAC-e Research Grant "Algebraic Multi-Grid Methods for Modeling and Simulation of Carbon Sequestration Processes on Multi-Core/GPU Architectures," No. 10-014677), and the Army Research Office under grant W911NF-13-1-0452. M.F. Wheeler and S. Lee would also like to thank the Affiliates of the Center for Subsurface Modeling at The University of Texas at Austin, Aramco, and Statoil. A. Mikelić would like to thank the Center for Subsurface Modeling, Institute for Computational Engineering and Science (ICES), and UT Austin for hospitality in April-May, 2016. His research was partially supported by the J. Tinsley Oden Faculty Fellowship Research Program and by the MOCOMIPOC project (Modélisation multiéchelles des écoulements complexes en présence de gaz dans les milieux chargés) from the NEEDS program (Projet fédérateur Milieux Poreux MIPOR), part of CNRS, France.

\section{References}

[1] Z. Chen, G. Huan, Y. Ma, Computational methods for multiphase flows in porous media, volume 2, SIAM, 2006.

[2] D. W. Peaceman, Improved treatment of dispersion in numerical calculation of multidimensional miscible displacement, Society of Petroleum Engineers Journal 6 (1966) 213-216. 


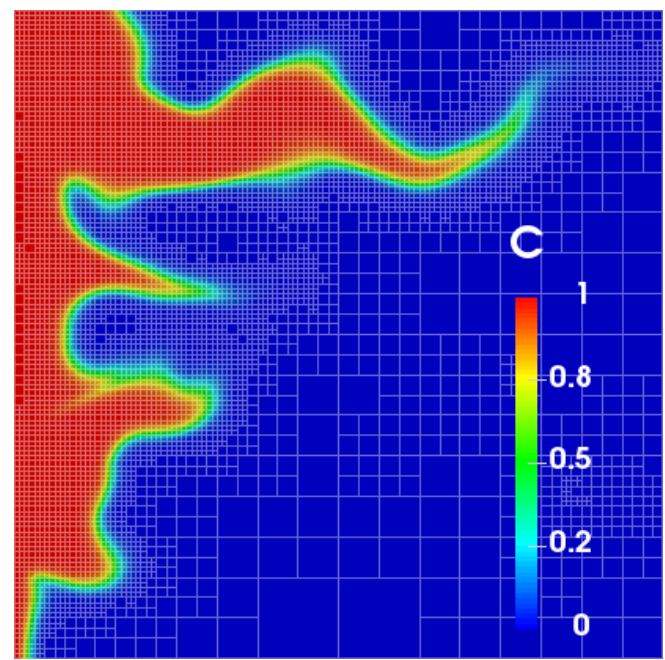

(a) Concentration values

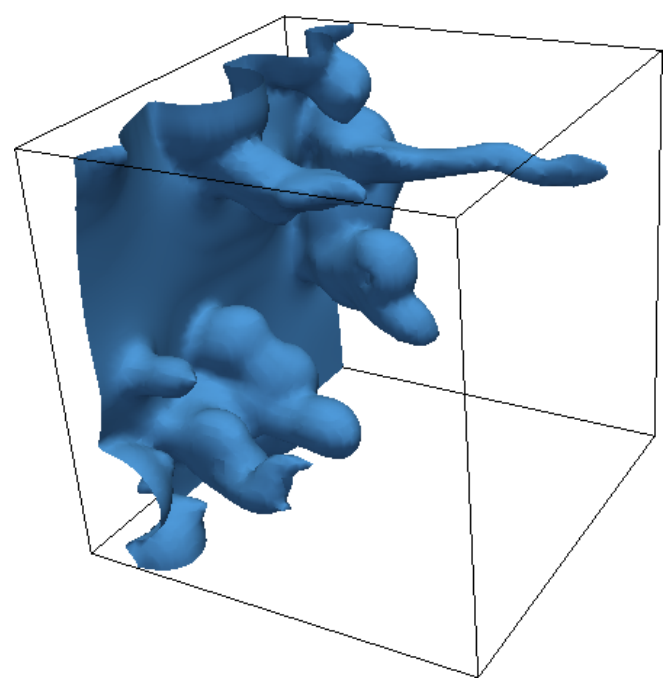

(b) Concentration value $(c=0.5)$

Figure 19: Two- and three-dimensional examples of unstable miscible displacements in heterogenous porous media of random permeability. For more details, see [174].

[3] P. Fabrie, M. Langlais, Mathematical analysis of miscible displacement in porous medium, SIAM Journal on Mathematical Analysis 23(6) (1992) 1375-1392.

[4] C. Tan, G. Homsy, Stability of miscible displacements in porous media: Rectilinear flow, Physics of Fluids 29 (1986) 3549-3556.

[5] C. Tan, G. Homsy, Simulation of nonlinear viscous fingering in miscible displacement, Physics of Fluids 31 (1988) 1330-1338.

[6] C. Tan, G. Homsy, Viscous fingering with permeability heterogeneity, Physics of Fluids A: Fluid Dynamics 4 (1992) 1099-1101.

[7] C. Tan, G. Homsy, Stability of miscible displacements in porous media: Radial source flow, Physics of Fluids 30 (1987) 1239-1245.

[8] O. Manickam, G. Homsy, Simulation of viscous fingering in miscible displacements with nonmonotonic viscosity profiles, Physics of Fluids 6 (1994) 95-107.

[9] G. M. Homsy, Viscous fingering in porous media, Annual Review of Fluid Mechanics 19 (1987) 271-311.

[10] W. Zimmerman, G. Homsy, Three-dimensional viscous fingering: A numerical study, Physics of Fluids A: Fluid Dynamics 4 (1992) 1901-1914.

[11] W. Zimmerman, G. Homsy, Viscous fingering in miscible displacements: Unification of effects of viscosity contrast, anisotropic dispersion, and velocity dependence of dispersion on nonlinear finger propagation, Physics of Fluids A: Fluid Dynamics 4 (1992) 2348-2359.

[12] W. Zimmerman, G. Homsy, Nonlinear viscous fingering in miscible displacement with anisotropic dispersion, Physics of Fluids A: Fluid Dynamics 3 (1991) 1859-1872.

[13] U. G. Araktingi, F. M. Orr Jr, Viscous fingering in heterogeneous porous media, SPE Advanced Technology Series 1 (1993) 71-80.

[14] C.-Y. Chen, E. Meiburg, Miscible porous media displacements in the quarter five-spot configuration. Part 1. The homogeneous case, Journal of Fluid Mechanics 371 (1998) 233-268.

[15] C.-Y. Chen, E. Meiburg, Miscible porous media displacements in the quarter five-spot configuration. Part 2. Effect of heterogeneities, Journal of Fluid Mechanics 371 (1998) 269-299.

[16] E. Meiburg, C.-Y. Chen, High-accuracy implicit finite-difference simulations of homogeneous and heterogeneous miscible-porous-medium flows, SPE Journal 5 (2000) 129-137.

[17] A. Riaz, H. A. Tchelepi, Linear stability analysis of immiscible two-phase flow in porous media with capillary dispersion and density variation, Physics of Fluids 16 (2004) 4727-4737.

[18] A. Riaz, H. A. Tchelepi, Numerical simulation of immiscible two-phase flow in porous media, Physics of Fluids 18 (2006) 014104.

[19] H. A. Tchelepi, F. M. Orr Jr., N. Rakotomalala, D. Salin, R. Woumeni, Dispersion, permeability heterogeneity, and viscous fingering: Acoustic experimental observations and particle tracking simulations, Physics of Fluids A: Fluid Dynamics 5 (1993) 1558-1574.

[20] P. G. Saffman, G. Taylor, The penetration of a fluid into a porous medium or Hele-Shaw cell containing a more viscous liquid, in: Proceedings of the Royal Society of A: Mathematical, Physical and Engineering Sciences. Vol. 245. The Royal Society, 1958, pp. $312-329$.

[21] P. G. Saffman, Viscous fingering in Hele-Shaw cells, Journal of Fluid Mechanics 173 (1986) 73-94.

[22] S. Chang, J. C. Slattery, A linear stability analysis for miscible displacements, Transport in Porous Media 1 (1986) 179-199.

[23] R. Raghavan, S. S. Marsden, The stability of immiscible liquid layers in a porous medium, Journal of Fluid Mechanics 48 (1971) 143-159.

[24] S. G. Shikaze, E. Sudicky, F. Schwartz, Density-dependent solute transport in discretely-fractured geologic media: is prediction possible?, Journal of Contaminant Hydrology 34 (1998) 273-291.

[25] R. A. Schincariol, F. W. Schwartz, C. A. Mendoza, Instabilities in variable density flows: stability and sensitivity analyses for homogeneous and heterogeneous media, Water Resources Research 33 (1997) 31-41.

[26] H. Zhang, F. W. Schwartz, Multispecies contaminant plumes in variable density flow systems, Water Resources Research 31 (1995) 

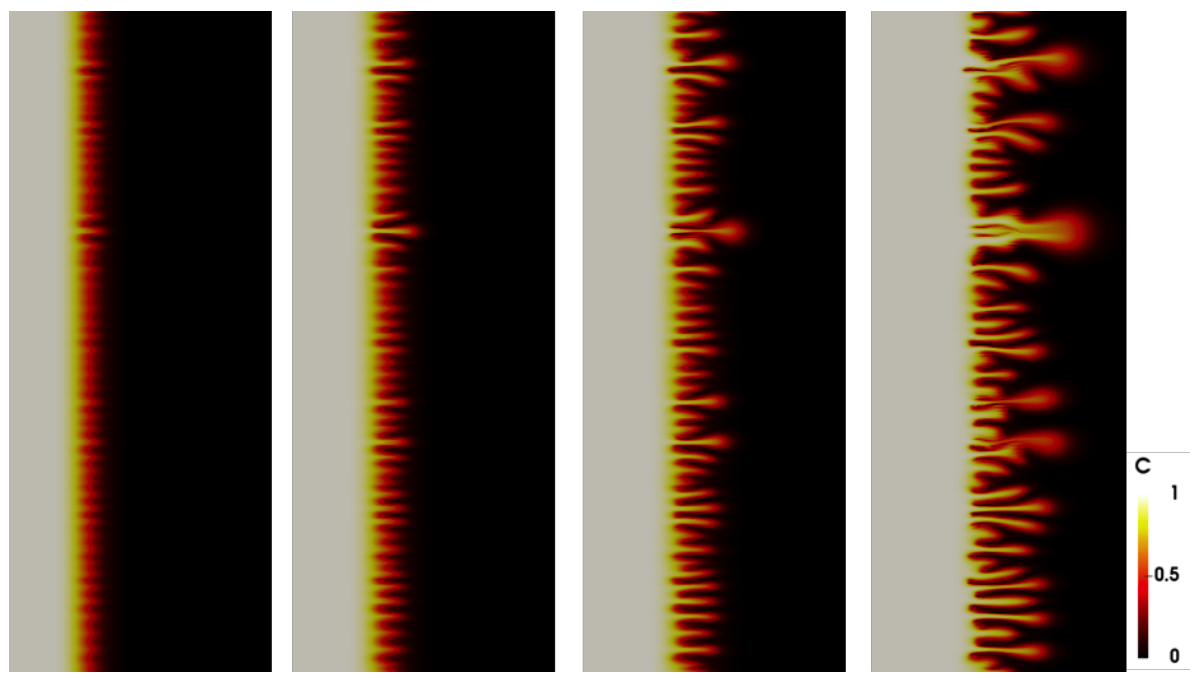

(a) Concentration values at $t=3.5,4,4.5$ and 5 .

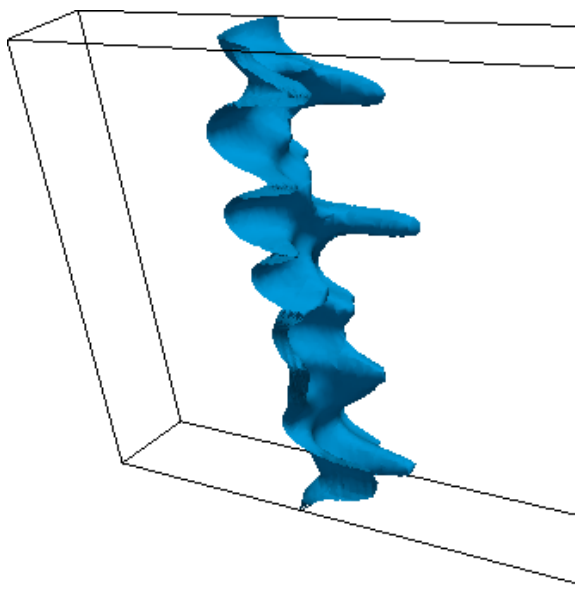

(b) Concentration values at $t=1.2 \mathrm{~s}$

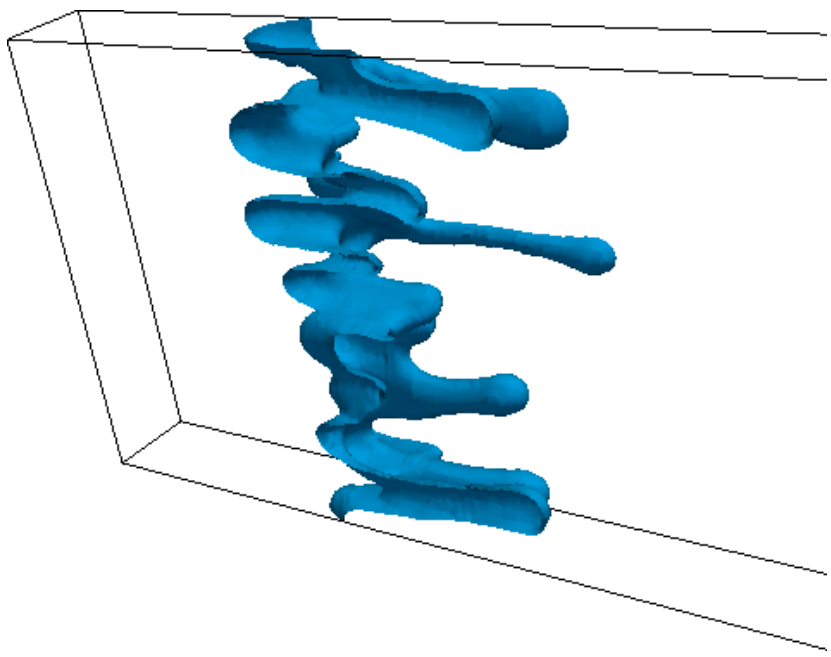

(c) Concentration values at $t=1.5 \mathrm{~s}$

Figure 20: Viscous fingering in Hele-Shaw cells. (a) Snapshots of concentration contours for a two-dimensional domain with mobility ratio 150. (b)-(c) Contour of $C=0.5$ for a three-dimensional calculation with mobility ratio 50 at two different time steps. For more details, see [174].

$837-847$.

[27] R. A. Schincariol, F. W. Schwartz, An experimental investigation of variable density flow and mixing in homogeneous and heterogeneous media, Water Resources Research 26 (1990) 2317-2329.

[28] D. Cuthiell, G. Kissel, C. Jackson, T. Frauenfeld, D. Fisher, K. Rispler, Viscous fingering effects in solvent displacement of heavy oil, J. Can. Pet. Tech. 45 (2006) 07.

[29] J. Sharma, S. B. Inwood, A. Kovscek, Experiments and analysis of multiscale viscous fingering during forced imbibition, SPE Journal 17 (2012) 1-142.

[30] L. Holm, Miscible displacement, Petroleum Engineering Hand Book, Society of Petroleum Engineers, Richardson, TX (1987) 1-45.

[31] J. Bear, Y. Bachmat, Introduction to modeling of transport phenomena in porous media, volume 4, Springer Science \& Business Media, 2012

[32] D. A. Nield, A. Bejan, Convection in porous media, Springer Science \& Business Media, 2006.

[33] O. Kolditz, R. Ratke, H.-J. G. Diersch, W. Zielke, Coupled groundwater flow and transport: 1. Verification of variable density flow and transport models, Advances in Water Resources 21 (1998) 27-46.

[34] H.-J. Diersch, O. Kolditz, Variable-density flow and transport in porous media: Approaches and challenges, Advances in Water Resources 25 (2002) 899-944.

[35] J. J. Hidalgo, J. Carrera, Effect of dispersion on the onset of convection during $\mathrm{CO}_{2}$ sequestration, Journal of Fluid Mechanics 640 (2009) 
$441-452$.

[36] G. S. H. Pau, J. B. Bell, K. Pruess, A. S. Almgren, M. J. Lijewski, K. Zhang, C. Yang, Y. Gu, High-resolution simulation and characterization of density-driven flow in $\mathrm{CO}_{2}$ storage in saline aquifers, Advances in Water Resources 33 (2010) 443-455.

[37] D. W. Peaceman, Fundamentals of numerical reservoir simulation, 1977.

[38] J. Douglas Jr, J. E. Roberts, Numerical methods for a model for compressible miscible displacement in porous media, Mathematics of Computation 41 (1983) 441-459.

[39] C. Choquet, Parabolic and degenerate parabolic models for pressure-driven transport problems, Mathematical Models and Methods in Applied Sciences 20 (2010) 543-566.

[40] Y. Amirat, K. Hamdache, A. Ziani, Mathematical analysis for compressible miscible displacement models in porous media, Mathematical Models and Methods in Applied Sciences 6 (1996) 729-747.

[41] H. Huang, R. Wattenbarger, X. Gai, W. P. Brown, O. J. Hehmeyer, J. Wang, T. A. Long, Using a fully coupled flow and geomechanical simulator to model injection into heavy oil reservoirs, volume 71, Wiley Online Library, 2013.

[42] A. Farina, A. Fasano, A. Mikelić, On the equations governing the flow of mechanically incompressible, but thermally expansible, viscous fluids, Mathematical Models and Methods in Applied Sciences 18 (2008) 813-857.

[43] S. N. Antontsev, A. Kazhiktov, V. N. Monakhov, Boundary value problems in mechanics of nonhomogeneous fluids, volume 22, Elsevier, 1989.

[44] I. Aganović, A. Mikelić, Homogenization of nonstationary flow of a two-constituant mixture through a porous medium, Asymptotic analysis 6 (1992) 173-189.

[45] A. Mikelić, I. Aganović, Homogenization of stationary flow of miscible fluids in a domain with a grained boundary, SIAM Journal on Mathematical Analysis 19 (1988) 287-294.

[46] A. Mikelić, Mathematical theory of stationary miscible filtration, Journal of Differential Equations 90 (1991) $186-202$.

[47] A. Mikelić, Regularity and uniqueness results for two-phase miscible flows in porous media, Flow in Porous Media (1993) $139-154$.

[48] M. Balhoff, A. Mikelić, M. F. Wheeler, Polynomial filtration laws for low Reynolds number flows through porous media, Transport in Porous Media 81 (2010) 35-60.

[49] M. Firdaouss, J. L. Guermond, P. Le Quéré, Nonlinear corrections to Darcy's law at low Reynolds numbers, Journal of Fluid Mechanics 343 (1997) 331-350.

[50] E. Marušić-Paloka, A. Mikelić, The derivation of a nonlinear filtration law including the inertia effects via homogenization, Nonlinear Analysis: Theory, Methods and Applications 42 (2000) 97-137.

[51] C. C. Mei, J. L. Auriault, The effect of weak inertia on flow through a porous medium, Journal of Fluid Mechanics 222 (1991) $647-663$.

[52] W. E, B. Engquist, X. Li, W. Ren, E. Vanden-Eijnden, Heterogeneous multiscale methods: A review, Communications on Computational Physics 2 (2007) 367-450.

[53] X. Yue, W. E, Numerical methods for multiscale transport equations and application to two-phase porous media flow, Journal of Computational Physics 210 (2005) 656-675.

[54] R. Li, N., X. L., Yue, Application and validation of an upscaling method for unsaturated water flow processes in heterogeneous soils, Vadose Zone Journal 14 (2015).

[55] P. Henning, M. Ohlberger, B. Schweizer, Adaptive heterogeneous multiscale methods for immiscible two-phase flow in porous media, Computational Geosciences 19 (2015) 99-114.

[56] Y. Efendiev, T. Y. Hou, Multiscale finite element methods: theory and applications, volume 4, Springer Science \& Business Media, 2009.

[57] Y. Efendiev, V. Ginting, T. Hou, R. Ewing, Accurate multiscale finite element methods for two-phase flow simulations, Journal of Computational Physics 220 (2006) 155-174.

[58] I. Lunati, P. Jenny, Multiscale finite-volume method for density-driven flow in porous media, Computational Geosciences 12 (2008) $337-350$.

[59] V. Ginting, Computational Upscaled Modeling of Heterogeneous Porous Media Flow Utilizing Finite Volume Method, Ph.D. thesis, Texas A \& M University, 2004.

[60] L. J. Durlofsky, Coarse scale models of two phase flow in heterogeneous reservoirs: Volume averaged equations and their relationship to existing upscaling techniques, Computational Geosciences 2 (1998) 73-92.

[61] Y. Efendiev, L. J. Durlofsky, S. H. Lee, Modeling of subgrid effects in coarse-scale simulations of transport in heterogeneous porous media, Water Resources Research 36 (2000) 2031-2041.

[62] P. Drazin, Introduction to Hydrodynamic Stability, volume 32, Cambridge University Press, 2002.

[63] P. G. Drazin, W. H. Reid, Hydrodynamic stability, Cambridge university press, 2004.

[64] V. I. I_Udovich, The linearization method in hydrodynamical stability theory, volume 74, American Mathematical Soc., 1989.

[65] J. Wloka, Partial Differential Equations, Cambridge University Press, 1987.

[66] G. Coskuner, R. G. Bentsen, An extended theory to predict the onset of viscous instabilities for miscible displacements in porous media, Transport in Porous Media 5 (1990) 473-490.

[67] E. Lajeunesse, J. Martin, N. Rakotomalala, D. Salin, 3D instability of miscible displacements in a Hele-Shaw cell, Physical review letters 79 (1997) 5254.

[68] E. Lajeunesse, J. Martin, N. Rakotomalala, D. Salin, Y. Yortsos, Miscible displacement in a Hele-Shaw cell at high rates, Journal of Fluid Mechanics 398 (1999) 299-319.

[69] M. d'Olce, J. Martin, N. Rakotomalala, D. Salin, L. Talon, Pearl and mushroom instability patterns in two miscible fluids' core annular flows, Physics of Fluids 20 (2008) 024104.

[70] A. Riaz, E. Meiburg, Radial source flows in porous media: Linear stability analysis of axial and helical perturbations in miscible displacements, Physics of Fluids 15 (2003) 938-946.

[71] Y. Yortsos, Stability of displacement processes in porous media in radial flow geometries, Physics of Fluids 30 (1987) $2928-2935$.

[72] M. Christie, D. Bond, Detailed simulation of unstable processes in miscible flooding, SPE Reservoir Engineering 2 (1987) 514-522.

[73] A. DeGregoria, A predictive monte carlo simulation of two-fluid flow through porous media at finite mobility ratio, Physics of Fluids 28 
(1985) 2933-2935.

[74] A. De Wit, G. Homsy, Viscous fingering in periodically heterogeneous porous media. I. Formulation and linear instability, The Journal of Chemical Physics 107 (1997) 9609-9618.

[75] A. De Wit, G. Homsy, Viscous fingering in periodically heterogeneous porous media. II. Numerical simulations, The Journal of Chemical Physics 107 (1997) 9619-9628.

[76] Y. C. Yortsos, M. Zeybek, Dispersion driven instability in miscible displacement in porous media, Physics of Fluids 31 (1988) $3511-3518$.

[77] F. Hickernell, Y. Yortsos, Linear stability of miscible displacement processes in porous media in the absence of dispersion, Studies in Applied Mathematics 74 (1986) 93-115.

[78] R. Balasubramaniam, N. Rashidnia, T. Maxworthy, J. Kuang, Instability of miscible interfaces in a cylindrical tube, Physics of Fluids 17 (2005) 052103.

[79] J. Scoffoni, E. Lajeunesse, G. Homsy, Interface instabilities during displacements of two miscible fluids in a vertical pipe, Physics of Fluids 13 (2001) 553-556.

[80] G. I. Taylor, Dispersion of soluble matter in solvent flowing slowly through a tube, in: Proceedings of the Royal Society of London A: Mathematical, Physical and Engineering Sciences, volume 219, The Royal Society, 1953, pp. 186-203.

[81] R. Aris, On the dispersion of a solute in a fluid flowing through a tube, in: Proceedings of the Royal Society of London A: mathematical, physical and engineering sciences, volume 235, The Royal Society, 1956, pp. 67-77.

[82] R. Caflisch, J. Rubinstein, Lectures on the mathematical theory of multiphase-flow, Courant Institute of Mathematical Sciences, New York, 1984.

[83] G. Mercer, A. Roberts, A centre manifold description of contaminant dispersion in channels with varying flow properties, SIAM Journal on Applied Mathematics 50 (1990) 1547-1565.

[84] S. Rosencrans, Taylor dispersion in curved channels, SIAM Journal on Applied Mathematics 57 (1997) 1216-1241.

[85] A. Mikelić, V. Devigne, C. J. Van Duijn, Rigorous upscaling of the reactive flow through a pore, under dominant Péclet and Damkohler numbers, SIAM Journal on Mathematical Analysis 38 (2006) 1262-1287.

[86] A. Mikelić, C. Rosier, Rigorous upscaling of the infinite adsorption rate reactive flow under dominant Péclet number through a pore, Annali dell'Università di Ferrara 53 (2007) 333-359.

[87] C. J. Van Duijn, A. Mikelić, I. S. Pop, C. Rosier, Effective dispersion equations for reactive flows with dominant Péclet and Damkohler numbers, Advances in Chemical Engineering 34 (2008) 1-45.

[88] C. Choquet, A. Mikelić, Laplace transform approach to the rigorous upscaling of the infinite adsorption rate reactive flow under dominant Péclet number through a pore, Applicable Analysis 87 (2008) 1373-1395.

[89] C. Choquet, A. Mikelić, Rigorous upscaling of the reactive flow with finite kinetics and under dominant Péclet number, Continuum mechanics and thermodynamics 21 (2009) 125-140.

[90] J. Rubinstein, R. Mauri, Dispersion and convection in periodic porous media, SIAM Journal on Applied Mathematics 46 (1986) 1018-1023

[91] G. I. Taylor, The dispersion of matter in turbulent flow through a pipe, in: Proceedings of the Royal Society of London A: Mathematical, Physical and Engineering Sciences, volume 223, The Royal Society, 1954, pp. 446-468.

[92] G. E. Karniadakis, A. Beskok, N. Aluru, Microflows and nanoflows: Fundamentals and simulation, volume 29, Springer Science \& Business Media, 2006.

[93] A. Scheidegger, Typical solutions of the differential equations of statistical theories of flow through porous media, Eos, Transactions American Geophysical Union 39 (1958) 929-932.

[94] J. Camacho, Thermodynamics of Taylor dispersion: Constitutive equations, Physical Review E 47 (1993) 1049.

[95] J. Camacho, Purely global model for Taylor dispersion, Physical Review E 48 (1993) 310.

[96] J. Camacho, Thermodynamic functions for Taylor dispersion, Physical Review E 48 (1993) 1844.

[97] C. Berentsen, M. Verlaan, C. Van Kruijsdijk, Upscaling and reversibility of Taylor dispersion in heterogeneous porous media, Physical Review E 71 (2005) 046308

[98] H.-C. Chang, V. Balakotaiah, Hyperbolic homogenized models for thermal and solutal dispersion, SIAM Journal on Applied Mathematics 63 (2003) 1231-1258.

[99] S. Chakraborty, V. Balakotaiah, Spatially averaged multi-scale models for chemical reactors, Advances in Chemical Engineering 30 (2005) 205-297.

[100] A. Mikelić, C. J. Van Duijn, Rigorous derivation of a hyperbolic model for Taylor dispersion, Mathematical Models and Methods in Applied Sciences 21 (2011) 1095-1120.

[101] R. Carbonell, S. Whitaker, Dispersion in pulsed systems-II: Theoretical developments for passive dispersion in porous media, Chemical Engineering Science 38 (1983) 1795-1802.

[102] M. Paine, R. Carbonell, S. Whitaker, Dispersion in pulsed systems-I: Heterogenous reaction and reversible adsorption in capillary tubes, Chemical Engineering Science 38 (1983) 1781-1793.

[103] M. Quintard, S. Whitaker, Transport in ordered and disordered porous media: Volume-averaged equations, closure problems, and comparison with experiment, Chemical Engineering Science 48 (1993) 2537-2564.

[104] C. C. Mei, Method of homogenization applied to dispersion in porous media, Transport in porous media 9 (1992) 261-274.

[105] J.-L. Auriault, P. Adler, Taylor dispersion in porous media: Analysis by multiple scale expansions, Advances in Water Resources 18 (1995) $217-226$.

[106] J. Salles, J.-F. Thovert, R. Delannay, L. Prevors, J.-L. Auriault, P. Adler, Taylor dispersion in porous media. Determination of the dispersion tensor, Physics of Fluids A: Fluid Dynamics (1989-1993) 5 (1993) 2348-2376.

[107] D. Edwards, M. Shapiro, H. Brenner, M. Shapira, Dispersion of inert solutes in spatially periodic, two-dimensional model porous media, Transport in Porous Media 6 (1991) 337-358.

[108] W. Perrins, D. McKenzie, R. McPhedran, Transport properties of regular arrays of cylinders, in: Proceedings of the Royal Society of London A: Mathematical, Physical and Engineering Sciences, volume 369, The Royal Society, 1979, pp. 207-225.

[109] D. L. Koch, R. G. Cox, H. Brenner, J. F. Brady, The effect of order on dispersion in porous media, Journal of Fluid Mechanics 200 (1989) 
$173-188$.

[110] G. Allaire, A.-L. Raphael, Homogenization of a convection-diffusion model with reaction in a porous medium, Comptes Rendus Mathematique 344 (2007) 523-528.

[111] G. Allaire, A. Mikelić, A. Piatnitski, Homogenization approach to the dispersion theory for reactive transport through porous media, SIAM Journal on Mathematical Analysis 42 (2010) 125-144.

[112] G. Allaire, R. Brizzi, A. Mikelić, A. Piatnitski, Two-scale expansion with drift approach to the Taylor dispersion for reactive transport through porous media, Chemical Engineering Science 65 (2010) 2292-2300.

[113] G. C. Papanicolaou, Diffusion in random media, in: Surveys in applied mathematics, Springer, 1995, pp. 205-253.

[114] C. J. Van Duijn, P. Knabner, Travelling waves in the transport of reactive solutes through porous media: Adsorption and binary ion exchange - Part 1, Transport in Porous Media 8 (1992) 167-194.

[115] P. Knabner, C. J. van Duijn, S. Hengst, An analysis of crystal dissolution fronts in flows through porous media. Part 1: Compatible boundary conditions, Advances in Water Resources 18 (1995) 171 - 185.

[116] E. Marušić-Paloka, A. L. Piatnitski, Homogenization of a nonlinear convection-diffusion equation with rapidly oscillating coefficients and strong convection, Journal of the London Mathematical Society 72 (2005) 391-409.

[117] B. Amaziane, A. Bourgeat, M. Jurak, Effective macrodiffusion in solute transport through heterogeneous porous media, Multiscale Modeling \& Simulation 5 (2006) 184-204.

[118] A. Bourgeat, M. Jurak, A. Piatnitski, Averaging a transport equation with small diffusion and oscillating velocity, Mathematical methods in the applied sciences 26 (2003) 95-117.

[119] L. W. Gelhar, C. Welty, K. R. Rehfeldt, A critical review of data on field-scale dispersion in aquifers, Water Resources Research 28 (1992) 1955-1974.

[120] D. J. Goode, L. F. Konikow, Apparent dispersion in transient groundwater flow, Water Resources Research 26 (1990) $2339-2351$.

[121] R. N. Horne, F. Rodriguez, Dispersion in tracer flow in fractured geothermal systems, Geophys. Res. Lett 10 (1983) $289-292$.

[122] P. Petitjeans, C.-Y. Chen, E. Meiburg, T. Maxworthy, Miscible quarter five-spot displacements in a Hele-Shaw cell and the role of flowinduced dispersion, Physics of Fluids 11 (1999) 1705-1716.

[123] J. Fernandez, P. Kurowski, P. Petitjeans, E. Meiburg, Density-driven unstable flows of miscible fluids in a Hele-Shaw cell, Journal of Fluid Mechanics 451 (2002) 239-260.

[124] F. Graf, E. Meiburg, C. Haertel, Density-driven instabilities of miscible fluids in a Hele-Shaw cell: Linear stability analysis of the threedimensional stokes equations, Journal of Fluid Mechanics 451 (2002) 261-282.

[125] N. Goyal, E. Meiburg, Unstable density stratification of miscible fluids in a vertical Hele-Shaw cell: Influence of variable viscosity on the linear stability, Journal of Fluid Mechanics 516 (2004) 211-238.

[126] S. H. Vanaparthy, E. Meiburg, D. Wilhelm, Density-driven instabilities of miscible fluids in a capillary tube: Linear stability analysis, Journal of Fluid Mechanics 497 (2003) 99-121.

[127] M. Kaviany, Principles of heat transfer in porous media, Springer Science \& Business Media, 2012.

[128] H. I. Ene, D. Poliševski, Thermal flows in porous media, volume 1, Springer Science \& Business Media, 2012.

[129] H. I. Ene, D. Poliševski, Steady convection in a porous layer with translational flow, Acta mechanica 84 (1990) $13-18$.

[130] H. I. Ene, Effects of anisotropy on the free convection from a vertical plate embedded in a porous medium, Transport in Porous Media 6 (1991) 183-194.

[131] H. I. Ene, E. Sanchez-Palencia, On thermal equation for flow in porous media, International Journal of Engineering Science 20 (1982) 623-630.

[132] F. Fayers, F. Jouaux, H. Tchelepi, An improved macroscopic model for viscous fingering and its validation for 2D and 3D flows. 1. non-gravity flows, In Situ 18 (1994) 43-78.

[133] J. P. Ennis-King, L. Paterson, Role of convective mixing in the long-term storage of carbon dioxide in deep saline formations, Spe Journal 10 (2005) 349-356.

[134] J. Ennis-King, I. Preston, L. Paterson, Onset of convection in anisotropic porous media subject to a rapid change in boundary conditions, Physics of Fluids 17 (2005) 084107.

[135] M. A. Hesse, Mathematical modeling and multi scale simulation of $\mathrm{CO}_{2}$ storage in saline aquifers, Ph.D. thesis, Stanford University, 2008.

[136] E. Lindeberg, D. Wessel-Berg, Vertical convection in an aquifer column under a gas cap of $\mathrm{CO}_{2}$, Energy Conversion and management 38 (1997) S229-S234.

[137] X. Xu, S. Chen, D. Zhang, Convective stability analysis of the long-term storage of carbon dioxide in deep saline aquifers, Advances in Water Resources 29 (2006) 397-407.

[138] A. Riaz, M. Hesse, H. A. Tchelepi, F. M. Orr Jr., Onset of convection in a gravitationally unstable diffusive boundary layer in porous media, Journal of Fluid Mechanics 548 (2006) 87-111.

[139] J. Gounot, J. P. Caltagirone, Stabilité et convection naturelle au sein d'une couche poreuse non homogène, International Journal of Heat and Mass Transfer 32 (1989) 1131-1140.

[140] J. J. Hidalgo, J. Carrera, A. Medina, Role of salt sources in density-dependent flow, Water Resources Research 45 (2009) W05503.

[141] G. S. Pau, J. B. Bell, K. Pruess, A. S. Almgren, M. J. Lijewski, K. Zhang, High-resolution simulation and characterization of density-driven flow in $\mathrm{CO}_{2}$ storage in saline aquifers, Advances in Water Resources 33 (2010) 443-455.

[142] G. S. H. Pau, A. S. Almgren, J. B. Bell, M. J. Lijewski, A parallel second-order adaptive mesh algorithm for incompressible flow in porous media, Phil. Trans. R. Soc. A 367 (2009) 4633-4654

[143] E. J. Koval, A method for predicting the performance of unstable miscible displacement in heterogeneous media, Society of Petroleum Engineers Journal 3 (1963) 145-154.

[144] M. Todd, P. O'dell, G. Hirasaki, Methods for increased accuracy in numerical reservoir simulators, Society of Petroleum Engineers Journal 12 (1972) 515-530.

[145] R. A. Wooding, Growth of fingers at an unstable diffusing interface in a porous medium or Hele-Shaw cell, Journal of Fluid Mechanics 39 (1969) 03. 
[146] G. Menon, F. Otto, Dynamic scaling in miscible viscous fingering, Communications in Mathematical Physics 257 (2005) $303-317$.

[147] O. Manickam, G. Homsy, Fingering instabilities in vertical miscible displacement flows in porous media, Journal of Fluid Mechanics 288 (1995) 75-102.

[148] D. A. DiCarlo, Experimental measurements of saturation overshoot on infiltration, Water Resources Research 40 (2004).

[149] C. J. Van Duijn, L. A. Peletier, I. S. Pop, A new class of entropy solutions of the buckley-leverett equation, SIAM Journal on Mathematical Analysis 39 (2007) 507-536.

[150] G. Menon, F. Otto, Fast communication: Diffusive slowdown in miscible viscous fingering, Communications in Mathematical Sciences 4 (2006) 267-273.

[151] Y. C. Yortsos, D. Salin, On the selection principle for viscous fingering in porous media, Journal of Fluid Mechanics 557 (2006) $225-236$

[152] Y. Yortsos, A theoretical analysis of vertical flow equilibrium, Transport in Porous Media 18 (1995) $107-129$.

[153] T. F. Russell, M. F. Wheeler, Finite element and finite difference methods for continuous flows in porous media, The mathematics of reservoir simulation 1 (1983) 35-106.

[154] R. E. Ewing, T. F. Russell, Efficient time-stepping methods for miscible displacement problems in porous media, SIAM Journal on Numerical Analysis 19 (1982) 1-67.

[155] R. E. Ewing, M. F. Wheeler, Galerkin methods for miscible displacement problems in porous media, SIAM Journal on Numerical Analysis 17 (1980) 351-365.

[156] J. B. Bell, G. R. Shubin, M. F. Wheeler, Analysis of a new method for computing the flow of miscible fluids in a porous medium, SIAM Journal on Numerical Analysis 22 (1985) 1041-1050.

[157] H. Huang, G. Scovazzi, A high-order, fully coupled, upwind, compact discontinuous Galerkin method for modeling of viscous fingering in compressible porous media, Computer Methods in Applied Mechanics and Engineering 263 (2013) 169-187.

[158] G. Scovazzi, H. Huang, S. Collis, J. Yin, A fully-coupled upwind discontinuous Galerkin method for incompressible porous media flows: High-order computations of viscous fingering instabilities in complex geometry, Journal of Computational Physics 252 (2013) 86-108.

[159] G. Shubin, J. Bell, An analysis of the grid orientation effect in numerical simulation of miscible displacement, Computer Methods in Applied Mechanics and Engineering 47 (1984) 47-71.

[160] T. C. Potempa, Finite element methods for convection dominated transport problems, Ph.D. thesis, Rice University, 1982.

[161] T. J. Hughes, G. Engel, L. Mazzei, M. G. Larson, The continuous Galerkin method is locally conservative, Journal of Computational Physics 163 (2000) 467-488

[162] C. Dawson, S. Sun, M. F. Wheeler, Compatible algorithms for coupled flow and transport, Comput. Methods Appl. Mech. Engrg. 193 (2004) 2565-2580.

[163] S. Lee, Y.-J. Lee, M. F. Wheeler, Enriched Galerkin approximations for coupled flow and transport system, 2016. Manuscript submitted for publication.

[164] T. Y. Hou, X.-H. Wu, A multiscale finite element method for elliptic problems in composite materials and porous media, Journal of Computational Physics 134 (1997) $169-189$.

[165] B. Ayuso de Dios, M. Holst, Y. Zhu, L. Zikatanov, Multilevel preconditioners for discontinuous, Galerkin approximations of elliptic problems, with jump coefficients, Mathematics of Computation 83 (2014) 1083-1120.

[166] S. Lee, Y.-J. Lee, M. F. Wheeler, A locally conservative enriched Galerkin approximation and efficient solver for elliptic and parabolic problems., SIAM Journal on Scientific Computing 38 (2016) A1404-A1429.

[167] Y. Wang, H. Hajibeygi, H. A. Tchelepi, Algebraic multiscale solver for flow in heterogeneous porous media, Journal of Computational Physics 259 (2014) $284-303$.

[168] M. Tene, Y. Wang, H. Hajibeygi, Adaptive algebraic multiscale solver for compressible flow in heterogeneous porous media, Journal of Computational Physics 300 (2015) 679 - 694.

[169] M. Tene, M. Al Kobaisi, H. Hajibeygi, Algebraic multiscale solver for flow in heterogeneous fractured porous media, in: SPE Reservoir Simulation Symposium, Society of Petroleum Engineers, 2015.

[170] J. R. Natvig, K.-A. Lie, Fast computation of multiphase flow in porous media by implicit discontinuous Galerkin schemes with optimal ordering of elements, Journal of Computational Physics 227 (2008) 10108-10124.

[171] B. Riviere, M. Wheeler, A posteriori error estimates and mesh adaptation strategy for discontinuous Galerkin methods applied to diffusion problems, Computers \& Mathematics with Applications 46 (2003) 141-163.

[172] B. Riviere, Discontinuous Galerkin finite element methods for solving the miscible displacement problem in porous media, Ph.D. thesis, The University of Texas at Austin, 2000.

[173] S. Sun, M. F. Wheeler, A posteriori error estimation and dynamic adaptivity for symmetric discontinuous Galerkin approximations of reactive transport problems, Computer Methods in Applied Mechanics and Engineering 195 (2006) 632-652.

[174] S. Lee, M. F. Wheeler, Adaptive enriched Galerkin methods for miscible displacement problems with entropy residual stabilization, 2016. Manuscript submitted for publication at Journal of Computational Physics.

[175] K. Aziz, A. Settari, Petroleum reservoir simulation, Chapman \& Hall, 1979.

[176] A. Weiser, M. F. Wheeler, On convergence of block-centered finite differences for elliptic problems, SIAM Journal on Numerical Analysis 25 (1988) 351-375.

[177] A. A. Samarskii, Local one dimensional difference schemes on non-uniform nets, USSR Computational Mathematics and Mathematical Physics 3 (1963) 572-619.

[178] S. Gerschgorin, Fehlerabschätzung für das differenzenverfahren zur lösung partieller differentialgleichungen, ZAMM-Journal of Applied Mathematics and Mechanics/Zeitschrift für Angewandte Mathematik und Mechanik 10 (1930) 373-382.

[179] J. Bramble, B. Hubbard, V. Thomee, Convergence estimates for essentially positive type discrete Dirichlet problems, Mathematics of Computation 23 (1969) 695-709.

[180] J. Nitsche, J. C. Nitsche, Error estimates for the numerical solution of elliptic differential equations, Archive for Rational Mechanics and Analysis 5 (1960) 293-306.

[181] R. Kellogg, An error estimate for elliptic difference equations on a convex polygon, SIAM Journal on Numerical Analysis 3 (1966) 79-90. 
[182] J. H. Bramble, On the convergence of difference approximations for second order uniformly elliptic operators, Numerical Solution of Field Problems in Continuum Physics 2 (1970) 201.

[183] H.-O. Kreiss, T. A. Manteuffel, B. Swartz, B. Wendroff, A. White, Supra-convergent schemes on irregular grids, Mathematics of Computation 47 (1986) 537-554.

[184] P. Forsyth, P. Sammon, Quadratic convergence for cell-centered grids, Applied Numerical Mathematics 4 (1988) $377-394$.

[185] M. F. Wheeler, G. Xue, I. Yotov, A multipoint flux mixed finite element method on distorted quadrilaterals and hexahedra, Numerische Mathematik 121 (2012) 165-204.

[186] I. Aavatsmark, Comparison of monotonicity for some multipoint flux approximation methods, in: Finite Volumes for Complex Applications, volume 5, 2008, pp. 19-34.

[187] Z. Heinemann, C. Brand, M. Munka, Y. Chen, Modeling reservoir geometry with irregular grids, in: SPE Symposium on Reservoir Simulation, Society of Petroleum Engineers, 1989.

[188] R. J. LeVeque, Finite volume methods for hyperbolic problems, volume 31, Cambridge university press, 2002.

[189] Z. Cai, J. Douglas Jr, M. Park, Development and analysis of higher order finite volume methods over rectangles for elliptic equations, Adv. Comput. Math. 19 (2003) 3-33.

[190] L. Chen, A new class of high order finite volume methods for second order elliptic equations, SIAM Journal on Numerical Analysis 47 (2010) 4021-4043.

[191] Z. Chen, J. Wu, Y. Xu, Higher-order finite volume methods for elliptic boundary value problems, Advances in Computational Mathematics 37 (2012) 191-253.

[192] R. Eymard, T. Gallouët, R. Herbin, Finite volume methods, Handbook of numerical analysis 7 (2000) $713-1018$.

[193] I. Babuška, The finite element method with Lagrangian multipliers, Numerische Mathematik 20 (1973) 179-192.

[194] F. Brezzi, On the existence, uniqueness and approximation of saddle-point problems arising from Lagrangian multipliers, Revue française d'automatique, informatique, recherche opérationnelle. Analyse numérique 8 (1974) 129-151.

[195] P.-A. Raviart, J.-M. Thomas, A mixed finite element method for 2-nd order elliptic problems, in: Mathematical aspects of finite element methods, Springer, 1977, pp. 292-315.

[196] J.-C. Nédélec, Mixed finite elements in $R^{3}$, Numerische Mathematik 35 (1980) 315-341.

[197] F. Brezzi, J. Douglas Jr, L. D. Marini, Two families of mixed finite elements for second order elliptic problems, Numerische Mathematik 47 (1985) 217-235.

[198] F. Brezzi, J. Douglas Jr, M. Fortin, L. D. Marini, Efficient rectangular mixed finite elements in two and three space variables, RAIROModélisation mathématique et analyse numérique 21 (1987) 581-604.

[199] F. Brezzi, J. Douglas Jr, R. Durán, M. Fortin, Mixed finite elements for second order elliptic problems in three variables, Numerische Mathematik 51 (1987) 237-250.

[200] Z. Chen, J. Douglas Jr, Prismatic mixed finite elements for second order elliptic problems, Calcolo 26 (1989) $135-148$.

[201] J. Warren, H. Price, F. Skiba, R. Varga, Miscible displacement: The liquid-liquid case, in: Fall Meeting of the Society of Petroleum Engineers of AIME, Society of Petroleum Engineers, 1961.

[202] H. Price, J. Cavendish, R. Varga, Numerical methods of higher-order accuracy for diffusion-convection equations, Society of Petroleum Engineers Journal 8 (1968) 293-303.

[203] A. Settari, H. Price, T. Dupont, Development and application of variational methods for simulation of miscible displacement in porous media, Society of Petroleum Engineers Journal 17 (1977) 228-246.

[204] L. C. Young, A finite-element method for reservoir simulation, Society of Petroleum Engineers Journal 21 (1981) 115-128.

[205] R. E. Ewing, M. F. Wheeler, Galerkin methods for miscible displacement problems with point sources and sinks-unit mobility ratio case, in: K. I. Gross (Ed.), Mathematical Methods in Energy Research, Society for Industrial and Applied Mathematics, Philadelphia, 1984, pp. $40-58$.

[206] J. D. Jr, M. F. Wheeler, B. L. Darlow, R. P. Kendall, Special issue on oil reservoir simulation self-adaptive finite element simulation of miscible displacement in porous media, Computer Methods in Applied Mechanics and Engineering 47 (1984) 131 - 159.

[207] P. H. Sammon, Numerical approximations for a miscible displacement process in porous media, SIAM Journal on Numerical Analysis 23 (1986) 508-542.

[208] A. Masud, T. J. Hughes, A stabilized mixed finite element method for darcy flow, Computer Methods in Applied Mechanics and Engineering 191 (2002) 4341-4370.

[209] J. Douglas Jr, T. Dupont, Interior penalty procedures for elliptic and parabolic Galerkin methods, in: Computing Methods in Applied Sciences, Springer, 1976, pp. 207-216.

[210] M. F. Wheeler, B. L. Darlow, Interior penalty Galerkin procedures for miscible displacement problems in porous media, Computational methods in nonlinear mechanics (1980) 485-506.

[211] J. Douglas Jr, M. F. Wheeler, B. L. Darlow, R. P. Kendall, Self-adaptive finite element simulation of miscible displacement in porous media, Computer Methods in Applied Mechanics and Engineering 47 (1984) 131-159.

[212] D. N. Arnold, F. Brezzi, B. Cockburn, L. D. Marini, Unified analysis of discontinuous Galerkin methods for elliptic problems, SIAM Journal on Numerical Analysis 39 (2002) 1749-1779.

[213] B. Riviere, M. F. Wheeler, V. Girault, A priori error estimates for finite element methods based on discontinuous approximation spaces for elliptic problems, SIAM Journal on Numerical Analysis 39 (2001) 902-931.

[214] S. Sun, M. F. Wheeler, Discontinuous Galerkin methods for coupled flow and reactive transport problems, Applied Numerical Mathematics 52 (2005) 273-298.

[215] S. Sun, M. F. Wheeler, Symmetric and nonsymmetric discontinuous Galerkin methods for reactive transport in porous media, SIAM Journal on Numerical Analysis 43 (2005) 195-219.

[216] F. Brezzi, G. Manzini, D. Marini, P. Pietra, A. Russo, Discontinuous finite elements for diffusion problems, in: Atti Convegno in onore di F. Brioschi (Milan, 1997), volume 16, 1999, pp. 197-217.

[217] T. J. R. Hughes, A. Masud, J. Wan, A stabilized mixed discontinuous Galerkin method for Darcy flow, Computer Methods in Applied 
Mechanics and Engineering 195 (2006) 3347-3381.

[218] F. Brezzi, T. J. R. Hughes, L. D. Marini, A. Masud, Mixed discontinuous Galerkin methods for Darcy flow, Journal of Scientific Computing 22 (2005) 119-145.

[219] F. Brezzi, T. J. Hughes, L. D. Marini, A. Masud, Mixed discontinuous Galerkin methods for darcy flow, Journal of Scientific Computing 22 (2005) 119-145.

[220] T. J. R. Hughes, G. Scovazzi, F. L. P., Multiscale and stabilized methods, in: E. Stein, R. de Borst, T. J. R. Hughes (Eds.), Encyclopedia of Computational Mechanics, John Wiley \& Sons, 2004.

[221] F. Brezzi, B. Cockburn, L. D. Marini, E. Süli, Stabilization mechanisms in discontinuous Galerkin finite element methods, Computer Methods in Applied Mechanics and Engineering 195 (2006) 3293-3310.

[222] J. Peraire, P.-O. Persson, The compact discontinuous Galerkin (CDG) method for elliptic problems, SIAM Journal on Scientific Computing 30 (2008) 1806-1824.

[223] B. Cockburn, C.-W. Shu, The local discontinuous Galerkin method for time-dependent convection-diffusion systems, SIAM Journal on Numerical Analysis 35 (1998) 2440-2463.

[224] S. Sun, J. Liu, A Locally Conservative Finite Element Method based on piecewise constant enrichment of the continuous Galerkin method, SIAM Journal on Scientific Computing 31 (2009) 2528-2548.

[225] R. J. LeVeque, Numerical methods for conservation laws, volume 132, Springer, 1992.

[226] S. K. Godunov, A difference method for numerical calculation of discontinuous solutions of the equations of hydrodynamics, Matematicheskii Sbornik 89 (1959) 271-306.

[227] B. Van Leer, Towards the ultimate conservative difference scheme. v. a second-order sequel to Godunov's method, Journal of computational Physics 32 (1979) 101-136.

[228] J. B. Bell, C. N. Dawson, G. R. Shubin., An unsplit higher order Godunov method for scalar conservation laws in multiple dimensions, Journal of Computational Physics 74.1 (1988) 1-24.

[229] J. Douglas Jr, Simulation of miscible displacement in porous media by a modified method of characteristic procedure, in: Numerical analysis., Springer Berlin Heidelberg, 1982, pp. 64-70.

[230] T. F. Russell, Finite elements with characteristics for two-component incompressible miscible displacement, in: SPE Reservoir Simulation Symposium, Society of Petroleum Engineers, 1982.

[231] R. Ewing, T. Russell, M. F. Wheeler, Simulation of miscible displacement using mixed methods and a modified method of characteristics, SPE Reservoir Simulation Symposium. Society of Petroleum Engineers (1983).

[232] T. F. Russell, M. F. Wheeler, C. Chiang, Large-scale simulation of miscible displacement by mixed and characteristic finite element methods, Mathematical and computational methods in seismic exploration and reservoir modeling (1986) 85-107.

[233] D. Moissis, Simulation of viscous fingering during miscible displacement in nonuniform porous media, Ph.D. thesis, Rice University, 1988.

[234] D. Moissis, M. Wheeler, C. Miller, Simulation of miscible viscous fingering using a modified method of characteristics: Effects of gravity and heterogeneity, SPE Advanced Technology Series 1 (1993) 62-70.

[235] J. Douglas Jr, T. F. Russell, Numerical methods for convection-dominated diffusion problems based on combining the method of characteristics with finite element or finite difference procedures, SIAM Journal on Numerical Analysis 19 (1982) 871-885.

[236] R. E. Ewing, T. F. Russell, M. F. Wheeler, Special issue on oil reservoir simulation convergence analysis of an approximation of miscible displacement in porous media by mixed finite elements and a modified method of characteristics, Computer Methods in Applied Mechanics and Engineering 47 (1984) 73-92.

[237] C. N. Dawson, T. F. Russell, M. F. Wheeler, Some improved error estimates for the modified method of characteristics, SIAM Journal on Numerical Analysis 26 (1989) 1487-1512.

[238] T. Arbogast, M. F. Wheeler, A characteristics-mixed finite element method for advection-dominated transport problems, SIAM Journal on Numerical Analysis 32 (1995) 404-424.

[239] T. Arbogast, C.-S. Huang, A fully mass and volume conserving implementation of a characteristic method for transport problems, SIAM Journal on Scientific Computing 28 (2006) 2001-2022.

[240] T. J. Hughes, Finite element methods for convection dominated flows, ASME, 1979. (Proceedings of the Annual Meeting of the American Society of Mechanical Engineers).

[241] A. F. Loula, E. L. Garcia, A. L. Coutinho, Miscible displacement simulation by finite element methods in distributed memory machines, Computer Methods in Applied Mechanics and Engineering 174 (1999) 339-354.

[242] A. L. Coutinho, J. L. Alves, Parallel finite element simulation of miscible displacements in porous media, SPE Journal 1 (1996) 487-500.

[243] A. L. Coutinho, J. L. Alves, Finite element simulation of nonlinear viscous fingering in miscible displacements with anisotropic dispersion and nonmonotonic viscosity profiles, Computational Mechanics 23 (1999) 108-116.

[244] A. Coutinho, C. Dias, J. Alves, L. Landau, A. Loula, S. Malta, R. Castro, E. Garcia, Stabilized methods and post-processing techniques for miscible displacements, Computer Methods in Applied Mechanics and Engineering 193 (2004) 1421-1436.

[245] A. N. Brooks, T. J. Hughes, Streamline upwind/Petrov-Galerkin formulations for convection dominated flows with particular emphasis on the incompressible navier-stokes equations, Computer Methods in Applied Mechanics and Engineering 32 (1982) 199-259.

[246] P. Lasaint, P.-A. Raviart, On a finite element method for solving the neutron transport equation, in: Mathematical aspects of finite elements in partial differential equations (Proc. Sympos., Math. Res. Center, Univ. Wisconsin, Madison, Wis., 1974), Math. Res. Center, Univ. of Wisconsin-Madison, Academic Press, New York, 1974, pp. 89-123. Publication No. 33.

[247] F. Brezzi, L. D. Marini, E. Süli, Discontinuous Galerkin methods for first-order hyperbolic problems, Mathematical Models and Methods in Applied Sciences 14 (2004) 1893-1903.

[248] B. Riviere, M. F. Wheeler, Discontinuous Galerkin methods for flow and transport problems in porous media, Communications in Numerical Methods in Engineering 18 (2002) 63-68.

[249] G. Scovazzi, A. Gerstenberger, S. S. Collis, A discontinuous Galerkin method for gravity-driven viscous fingering instabilities in porous media, Journal of Computational Physics (2012).

[250] A. Gerstenberger, G. Scovazzi, S. S. Collis, Computing gravity-driven viscous fingering flows in complex subsurface geometries: A high- 
order discontinuous Galerkin approach, Computational Geosciences (2012).

[251] J.-L. Guermond, R. Pasquetti, B. Popov, Entropy viscosity method for nonlinear conservation laws, Journal of Computational Physics 230 (2011) 4248-4267.

[252] A. Bonito, J.-L. Guermond, B. Popov, Stability analysis of explicit entropy viscosity methods for non-linear scalar conservation equations, Math. Comp. 83 (2014) 1039-1062.

[253] M. Delshad, X. Kong, R. Tavakoli, S. A. Hosseini, M. F. Wheeler, Modeling and simulation of carbon sequestration at cranfield incorporating new physical models, International Journal of Greenhouse Gas Control 18 (2013) 463 - 473.

[254] S. Rassenfoss, A Tricky Tradeoff-Can Adding a Little Solvent Yield a Lot More Heavy Crude?, volume 64(06), Society of Petroleum Engineers, 2012

[255] T. T. Boone, C. C. Wattenbarger, S. Clingman, J. L. Dickson, An integrated technology development plan for solvent-based recovery of heavy oil, SPE Heavy Oil Conference and Exhibition. Society of Petroleum Engineers (2011).

[256] R. K. Jha, M. Kumar, I. Benson, E. Hanzlik, New insights into steam/solvent-coinjection-process mechanism, SPE Journal 18 (2013) $867-877$.

[257] J. Dickson, L. Dittaro, T. Boone, Integrating the key learnings from laboratory, simulation, and field tests to assess the potential for solvent assisted-steam assisted gravity drainage, in: Paper SPE 165485 presented at 2013 SPE Heavy Oil Conference, Calgary, Alberta, Canada, 2013, pp. 11-13.

[258] D. McDuff, C. E. Shuchart, S. Jackson, D. Postl, J. S. Brown, Understanding wormholes in carbonates: Unprecedented experimental scale and 3-D visualization, SPE Annual Technical Conference and Exhibition. Society of Petroleum Engineers (2010).

[259] C. Shuchart, J. Alvarez, D. McDuff, D. Chang, A. Troshko, Advances in ExxonMobil's carbonate stimulation methodology-from wormholes to long-term productivity, International Petroleum Exhibition and Conference (2010) 1-4.

[260] P. Valsecchi, D. McDuff, D.-L. Chang, H. Huang, J. Burdette, T. A. Long, C. Karmonik, Simulation and visualization of near-well flow, in: SPE International Production and Operations Conference \& Exhibition, Society of Petroleum Engineers, 2012 , p. 15.

[261] A. Alghamdi, M. A. Nasr-El-Din, A. D. Hill, H. A. Nasr-El-Din, Diversion and propagation of viscoelastic surfactant based acid in carbonate cores, in: SPE International Symposium on Oilfield Chemistry, Society of Petroleum Engineers, 2009.

[262] M. G. Melendez, M. Pournik, D. Zhu, A. D. Hill, The effects of acid contact time and the resulting weakening of the rock surfaces on acid fracture conductivity, in: European Formation Damage Conference, Society of Petroleum Engineers, 2007.

[263] M. Saneifar, H. A. Nasr-El-Din, R. A. Nasralla, M. M. Fahes, D. HIll, Effect of spent acids on the wettability of sandstones and carbonates at high temperature and pressure, in: SPE European Formation Damage Conference, Society of Petroleum Engineers, 2011.

[264] M. Saneifar, M. M. Fahes, D. HIll, B. Zoghbi, N. Sheikh Ali, An experimental investigation of carbonate rock wettability after stimulation fluids invasion, in: SPE EUROPEC/EAGE Annual Conference and Exhibition, Society of Petroleum Engineers, 2011.

[265] L. N. Morgenthaler, D. Zhu, J. Mou, A. D. Hill, Effect of reservoir mineralogy and texture on acid response in heterogeneous sandstones, in: SPE Annual Technical Conference and Exhibition, Society of Petroleum Engineers, 2006.

[266] M. J. Economides, A. D. Hill, C. Ehlig-Economides, D. Zhu, Petroleum production systems, Pearson Education, 2012.

[267] F. Stalkup, Miscible displacement, Monograph (Society of Petroleum Engineers of AIME), Henry L. Doherty Memorial Fund of AIME, Society of Petroleum Engineers of AIME, 1983.

[268] H. A. Tchelepi, F. M. Orr Jr., Interaction of viscous fingering, permeability heterogeneity, and gravity segregation in three dimensions., SPE Reservoir Engineering 9 (1994) 266-271.

[269] H. Tchelepi, Viscous Fingering, Gravity Segregation and Permeability Heterogeneity in Two-dimensional and Three Dimensional Flows, Ph.D. thesis, Stanford University, 1994.

[270] M. Christie, A. Muggeridge, J. Barley, 3D simulation of viscous fingering and WAG schemes, SPE Reserv. Eng. 8 (1993) 01.

[271] T. A. Prickett, T. G. Naymik, C. G. Lonnquist, A random walk solute transport model for selected groundwater quality evaluations, Bulletin 651981.103 (1981) 68.

[272] W. Kinzelbach, The random walk method in pollutant transport simulation, in: Groundwater flow and quality modelling, Springer, 1988, pp. 227-245.

[273] Y.-B. Chang, M. Lim, G. Pope, S. K, $\mathrm{CO}_{2}$ flow patterns under multiphase flow: Heterogeneous field-scale conditions, SPE Reservoir Engineering 9 (1994) 208-216

[274] K. Mohanty, S. Johnson, Interpretation of laboratory gasfloods with multidimensional compositional modeling, SPE reservoir engineering 8 (1993) 59-66.

[275] J. Douglas Jr, R. E. Ewing, M. F. Wheeler, The approximation of the pressure by a mixed method in the simulation of miscible displacement, ESAIM: Mathematical Modeling and Numerical Analysis 17 (1983) 17-33.

[276] B. L. Darlow, R. E. Ewing, M. F. Wheeler, Mixed finite element method for miscible displacement problems in porous media, Society of Petroleum Engineers Journal 24 (1984) 391-398.

[277] S. Sun, B. Riviere, M. F. Wheeler, A combined mixed finite element and discontinuous Galerkin method for miscible displacement problem in porous media, in: Recent progress in computational and applied PDEs, Springer, 2002, pp. 323-351.

[278] J. Moortgat, S. Sun, A. Firoozabadi, Compositional modeling of three-phase flow with gravity using higher-order finite element methods, Water Resources Research 47 (2011).

[279] H. Hoteit, A. Firoozabadi, Multicomponent fluid flow by discontinuous Galerkin and mixed methods in unfractured and fractured media, Advances in Water Resources 41 (2005).

[280] M. F. Wheeler, Numerical simulation in oil recovery, Springer-Verlag New York Inc., New York, NY, 1988.

[281] J. Li, B. Riviere, Numerical solutions of the incompressible miscible displacement equations in heterogeneous media, Computer Methods in Applied Mechanics and Engineering 292 (2015) 107-121.

[282] J. Li, B. Riviere, High order discontinuous Galerkin method for simulating miscible flooding in porous media, Computational Geosciences 19 (2015) 1251-1268.

[283] A. Riaz, H. A. Tchelepi, Influence of relative permeability on the stability characteristics of immiscible flow in porous media, Transport in Porous Media 64 (2006) 315-338. 
[284] M. F. Wheeler, C. Dawson, An operator-splitting method for advection-diffusion-reaction problems, The Mathematics of Finite Elements and Applications VI (1987) 463-382.

[285] The Hanford Site, 2016. URL: http://www.hanford.gov/

[286] T. Arbogast, M. F. Wheeler, A parallel numerical model for subsurface contaminant transport with biodegradation kinetics, The Mathematics of Finite Elements and Applications (1994) 199-213.

[287] S. Sun, M. F. Wheeler, Anisotropic and dynamic mesh adaptation for discontinuous Galerkin methods applied to reactive transport, Computer Methods in Applied Mechanics and Engineering 195 (2006) 3382-3405.

[288] S. Sun, M. F. Wheeler, Discontinuous Galerkin methods for simulating bioreactive transport of viruses in porous media, Advances in Water Resources 30 (2007) 1696-1710.

[289] C. Y. Chiang, C. N. Dawson, M. F. Wheeler, Modeling of in-situ biorestoration of organic compounds in groundwater, Transport in Porous Media 6 (1991) 667-702.

[290] C. N. Dawson, C. J. V. Duijn, M. F. Wheeler, Characteristic-Galerkin methods for contaminant transport with nonequilibrium adsorption kinetics, SIAM Journal on Numerical Analysis 31 (1994) 982-999.

[291] C. A. Blyton, D. P. Gala, M. M. Sharma, A comprehensive study of proppant transport in a hydraulic fracture, in: SPE Annual Technical Conference and Exhibition, Society of Petroleum Engineers, 2015, p. 21.

[292] S. Malhotra, E. R. Lehman, M. M. Sharma, Proppant placement using alternate-slug fracturing, SPE Journal 19 (2014) 974-985.

[293] S. Lee, A. Mikelić, M. F. Wheeler, T. Wick, Phase-field modeling of proppant-filled fractures in a poroelastic medium, Computer Methods in Applied Mechanics and Engineering, 2016.

[294] S. Malhotra, M. M. Sharma, E. R. Lehman, Experimental study of the growth of mixing zone in miscible viscous fingering, Physics of Fluids 27 (2015) 014105.

[295] V. Zingan, J.-L. Guermond, J. Morel, B. Popov, Implementation of the entropy viscosity method with the discontinuous Galerkin method, Computer Methods in Applied Mechanics and Engineering 253 (2013) 479-490.

[296] A. Bonito, J.-L. Guermond, S. Lee, Numerical simulations of bouncing jets, International Journal for Numerical Methods in Fluids 80 (2016) 53-75. 